\title{
THE CALIBRATION OF MONOCHROMATIC FAR-INFRARED STAR FORMATION RATE INDICATORS*
}

\author{
D. Calzetti ${ }^{1}$, S.-Y. Wu ${ }^{1}$, S. Hong ${ }^{1}$, R. C. Kennicutt ${ }^{2}$, J. C. LeE $^{3}$, D. A. Dale ${ }^{4}$, C. W. Engelbracht ${ }^{5}$, L. van Zee ${ }^{6}$, \\ B. T. Draine ${ }^{7}$, C.-N. HaO ${ }^{2,8}$, K. D. Gordon ${ }^{9}$, J. Moustakas ${ }^{10}$, E. J. Murphy ${ }^{11}$, M. Regan ${ }^{9}$, A. Begum ${ }^{2}$, M. Block ${ }^{5}$, \\ J. Dalcanton ${ }^{12}$, J. Funes ${ }^{13}$, A. Gil de Paz ${ }^{14}$, B. Johnson ${ }^{2}$, S. Sakai ${ }^{15}$, E. Skillman ${ }^{16}$, F. Walter ${ }^{17}$, D. Weisz ${ }^{16}$, \\ B. Williams ${ }^{12}$, AND Y. Wu ${ }^{18}$ \\ ${ }^{1}$ Department of Astronomy, University of Massachusetts, Amherst, MA 01003, USA; calzetti@astro.umass.edu \\ ${ }^{2}$ Institute of Astronomy, Cambridge University, Cambridge, UK \\ ${ }^{3}$ Carnegie Observatories of Washington, Pasadena, CA, USA \\ ${ }^{4}$ Department of Physics and Astronomy, University of Wyoming, WY, USA \\ ${ }^{5}$ Steward Observatory, University of Arizona, AZ, USA \\ ${ }^{6}$ Department of Astronomy, University of Indiana, IN, USA \\ ${ }^{7}$ Princeton University Observatory, Peyton Hall, Princeton, NJ, USA \\ ${ }^{8}$ Tianjin Astrophysical Center, Tianjin Normal University, 300387 Tianjin, China \\ ${ }^{9}$ Space Telescope Science Institute, Baltimore, MD, USA \\ ${ }^{10}$ Center for Astrophysics and Space Sciences, University of California, San Diego, CA, USA \\ ${ }^{11}$ Spitzer Science Center, Caltech, CA, USA \\ 12 Department of Astronomy, University of Washington, WA, USA \\ ${ }^{13}$ Vatican Observatory, University of Arizona, AZ, USA \\ ${ }^{14}$ Departamento de Astrofisica, Universidad Computense de Madrid, Spain \\ ${ }^{15}$ Department of Astronomy, UCLA, CA, USA \\ ${ }^{16}$ Department of Astronomy, University of Minnesota, MN, USA \\ ${ }_{17}$ Max Planck Institut für Astronomie, Heidelberg, Germany \\ ${ }^{18}$ IPAC, CalTech, Pasadena, CA, USA \\ Received 2009 August 3; accepted 2010 March 3; published 2010 April 19
}

\begin{abstract}
Spitzer data at 24, 70, and $160 \mu \mathrm{m}$ and ground-based $\mathrm{H} \alpha$ images are analyzed for a sample of 189 nearby star-forming and starburst galaxies to investigate whether reliable star formation rate (SFR) indicators can be defined using the monochromatic infrared dust emission centered at 70 and $160 \mu \mathrm{m}$. We compare recently published recipes for SFR measures using combinations of the $24 \mu \mathrm{m}$ and observed H $\alpha$ luminosities with those using $24 \mu \mathrm{m}$ luminosity alone. From these comparisons, we derive a reference SFR indicator for use in our analysis. Linear correlations between SFR and the $70 \mu \mathrm{m}$ and $160 \mu \mathrm{m}$ luminosity are found for $L(70) \gtrsim 1.4 \times$ $10^{42} \mathrm{erg} \mathrm{s}^{-1}$ and $L(160) \gtrsim 2 \times 10^{42} \mathrm{erg} \mathrm{s}^{-1}$, corresponding to SFR $\gtrsim 0.1-0.3 M_{\odot} \mathrm{yr}^{-1}$, and calibrations of SFRs based on $L(70)$ and $L(160)$ are proposed. Below those two luminosity limits, the relation between SFR and $70 \mu \mathrm{m}(160 \mu \mathrm{m})$ luminosity is nonlinear and SFR calibrations become problematic. A more important limitation is the dispersion of the data around the mean trend, which increases for increasing wavelength. The scatter of the $70 \mu \mathrm{m}(160 \mu \mathrm{m})$ data around the mean is about $25 \%$ (factor 2 ) larger than the scatter of the $24 \mu \mathrm{m}$ data. We interpret this increasing dispersion as an effect of the increasing contribution to the infrared emission of dust heated by stellar populations not associated with the current star formation. Thus, the 70 (160) $\mu \mathrm{m}$ luminosity can be reliably used to trace SFRs in large galaxy samples, but will be of limited utility for individual objects, with the exception of infrared-dominated galaxies. The nonlinear relation between SFR and the 70 and $160 \mu \mathrm{m}$ emission at faint galaxy luminosities suggests a variety of mechanisms affecting the infrared emission for decreasing luminosity, such as increasing transparency of the interstellar medium, decreasing effective dust temperature, and decreasing filling factor of star-forming regions across the galaxy. In all cases, the calibrations hold for galaxies with oxygen abundance higher than roughly $12+\log (\mathrm{O} / \mathrm{H}) \sim 8.1$. At lower metallicity, the infrared luminosity no longer reliably traces the SFR because galaxies are less dusty and more transparent.
\end{abstract}

Key words: galaxies: interactions - galaxies: ISM - galaxies: starburst - infrared: galaxies - ISM: structure stars: formation

Online-only material: color figures

\section{INTRODUCTION}

The star formation rate (SFR) is one of the principal parameters that needs to be measured in star-forming regions and galaxies, in order to characterize their evolution. Extensive efforts have been made over the past couple of decades to derive SFR indicators from luminosities at a variety of wavelengths, spanning from the UV, where the recently formed mas-

\footnotetext{
* Based on observations obtained with the Spitzer Space Telescope, which is operated by JPL, CalTech, under NASA Contract 1407.
}

sive stars emit the bulk of their energy, to the infrared, where the dust-reprocessed light from those stars emerges, to the radio, which is mostly a tracer of supernova activity (e.g., Kennicutt 1998; Yun et al. 2001; Kewley et al. 2002; Ranalli et al. 2003; Hirashita et al. 2003; Bell 2003; Kewley et al. 2004; Calzetti et al. 2005, 2007; Schmitt et al. 2006; Moustakas et al. 2006; Alonso-Herrero et al. 2006; Salim et al. 2007; Persic \& Rephaeli 2007; Rosa-Gonzalez et al. 2007; Kennicutt et al. 2007; Bigiel et al. 2008; Rieke et al. 2009; Calzetti 2009, for earlier papers, see Kennicutt 1998). 
In recent years, investigations of monochromatic (i.e., based on a single band measurement) SFR indicators based on the infrared emission from galaxies have experienced a new resurgence, thanks to the high-sensitivity and high-angular-resolution data provided by the Spitzer Space Telescope, which have yielded both deeper distant galaxy surveys and more accurate information on the relation between dust and stellar emission in nearby galaxies. Deep surveys are often characterized by limited information across the infrared wavelength range and monochromatic SFRs are an important tool for these projects.

The rest-frame mid-infrared emission from dust in galaxies, in particular the emission detected in the $8 \mu \mathrm{m}$ and $24 \mu \mathrm{m}$ Spitzer bands, has been analyzed by a number of authors (Roussel et al. 2001; Förster Schreiber et al. 2004; Boselli et al. 2004; Calzetti et al. 2005, 2007; Wu et al. 2005; Alonso-Herrero et al. 2006; Perez-Gonzalez et al. 2006; Relaño et al. 2007; Zhu et al. 2008; Rieke et al. 2009; Salim et al. 2009), and a general correlation (but also a number of caveats) between mid-IR infrared emission and SFR has been found.

Over the next few years, new facilities, both from space (e.g., the Herschel Space Telescope) and from the ground (ALMA and the Large Millimeter Telescope, to mention just two) will open new windows of sensitivity at even longer wavelengths than those explored by Spitzer, and will, in turn, provide even more powerful tools for probing the evolution of the rate at which galaxies have assembled their gas and dust components. Deep surveys will be able to probe the dust emission from galaxies at rest-frame infrared wavelengths that are close to the peak emission, and to the bulk of the infrared energy budget. Herschel will, for instance, enable us to probe the peak dust emission from galaxies $(\sim 60-150 \mu \mathrm{m})$ up to redshift $z \sim 2$, while the Rayleigh-Jeans tail of the dust emission will be the dominion of submillimeter and millimeter facilities.

Exploring the viability, and limitations, of using the monochromatic emission close to the infrared peak and at longer wavelengths as SFR indicators is thus timely for providing a reference for those future surveys. In this paper, we investigate the use of the $70 \mu \mathrm{m}$ and $160 \mu \mathrm{m}$ emission, the two longest wavelength Spitzer bands, from nearby galaxies as SFR diagnostics.

The present paper is organized as follows: Section 2 introduces the sample of local star-forming galaxies and the data used for the present analysis; Section 3 describes the quantities used in this work; Section 4 compares a variety of existing SFR indicators in the optical/infrared, to derive "reference" SFRs for our galaxies, which are then compared with the Spitzer $70 \mu \mathrm{m}$ and $160 \mu \mathrm{m}$ emission from the same galaxies and with expectations from models in Sections 5 and 6, respectively. A discussion of the results and the conclusions are given in Section 7. Throughout the paper, we adopt a value of the Hubble constant $H_{0}=70 \mathrm{~km} \mathrm{~s}^{-1} \mathrm{Mpc}^{-1}$.

\section{SAMPLE DESCRIPTION}

We combine data from two Spitzer Legacy surveys, Local Volume Legacy (LVL; Dale et al. 2009; J. C. Lee et al. 2010, in preparation) and Spitzer Infrared Nearby Galaxies Survey (SINGS; Kennicutt et al. 2003), with the local starburst galaxy sample of the Spitzer MIPS-GTO programs (Engelbracht et al. 2008) and the luminous infrared galaxies (LIRGs) sample of Alonso-Herrero et al. (2006), in order to span close to 5 orders of magnitude in SFR surface density (SFSD $=\mathrm{SFR} /$ area), and thus cover as wide as possible a range that may characterize star-forming galaxies at all redshifts.
Galaxies with MIPS detections at 24, 70, and $160 \mu \mathrm{m}$ and $\mathrm{H} \alpha$ measurements are preferentially selected, though, in order to broaden the sample to low-luminosity and low-metallicity objects, galaxies with MIPS upper limits are included if $\mathrm{H} \alpha(\lambda 0.6563 \mu \mathrm{m})$ or $\mathrm{P} \alpha(\lambda 1.876 \mu \mathrm{m})$ data exist for them. In general, when both MIPS and $\mathrm{H} \alpha$ measurements are present, extinction-corrected SFRs will be derived by combining the observed $24 \mu \mathrm{m}$ and $\mathrm{H} \alpha$ luminosities (see Section 4.2); for the few MIPS $24 \mu \mathrm{m}$ upper limits in our sample, we will rely on the extinction-corrected (via the $\mathrm{H} \alpha / \mathrm{P} \alpha$ ratio) $\mathrm{P} \alpha$ luminosity, or will assume, for low-metallicity objects, that the SFR is practically unobscured by dust and use the observed $\mathrm{H} \alpha$ luminosity. We also require that measurements of oxygen abundance be available for each galaxy, either from the spectroscopy of J. Moustakas et al. (2010, in preparation) or from the literature (Marble et al. 2010; Engelbracht et al. 2008), as the infrared luminosity is sensitive to the metal abundance in galaxies (Cannon et al. 2005, 2006a, 2006b; Engelbracht et al. 2005; Walter et al. 2007; Calzetti et al. 2007; Relaño et al. 2007), and this is one of the parameters investigated in the present paper. Finally, images in the emission lines of $\mathrm{H} \alpha$ or $\mathrm{H} \alpha+[\mathrm{N}$ II] need to be available, to measure the extent of the emitting region in the warm ionized gas; we use the line-emitting area to normalize all luminosities, in order to remove any dependence on distance from our results, and to ensure that galaxies are not scaled by mass (or by global luminosity). In this paper, we use the definition of luminosity surface density (LSD = luminosity/area), and infrared luminosity surface density (IRSD), to remove any dependence of luminosity on the galaxy distance or size.

The selection criteria and observation strategy for the 258 galaxies in the LVL sample are described in Dale et al. (2009) and J. C. Lee et al. (2010, in preparation); IRAC and MIPS total fluxes for each galaxy are reported in Dale et al. (2009), while the foreground-galactic-extinction-corrected $\mathrm{H} \alpha+[\mathrm{N}$ II] total fluxes and $[\mathrm{N} \mathrm{II}] / \mathrm{H} \alpha$ ratios and other general information on the galaxies, including distances, are reported in Kennicutt et al. (2008). The main criterion for the LVL sample is that galaxies need to be within the local $11 \mathrm{Mpc}$ volume. Oxygen abundances from the literature are currently available for 108 of the LVL galaxies (Marble et al. 2010); the additional requirement to have narrow-band optical images available for measuring emission region sizes further reduces the sample to 80 galaxies. ${ }^{19}$ Of these, 11 are starbursts already included in the MIPS-GTO sample (see below); we thus consider our final LVL sample as consisting of 69 galaxies.

The SINGS sample contains 75 galaxies closer than about $30 \mathrm{Mpc}$, representative of a wide range of properties in terms of morphology, luminosity, dust temperature, etc. (Kennicutt et al. 2003). Of those 75 galaxies, 33 are in common with the LVL sample. The number of SINGS galaxies included in our sample is further reduced by excluding non-star-forming, early type galaxies, mostly Sy2-dominated ellipticals or S0's (NGC 584, NGC 855, NGC 1266, NGC 1316, NGC 1404, NGC 4552, and NGC 5195), and one galaxy, NGC 5033, for which optical narrow-band data are not available. We thus have a final sample of 67 SINGS galaxies, 31 of which in common with the LVL sample. Total MIPS fluxes for the SINGS galaxies are listed in Dale et al. (2007), and the $\mathrm{H} \alpha+[\mathrm{N}$ II] fluxes plus the [N II] $/ \mathrm{H} \alpha$ ratios are listed in Kennicutt et al. (2009). Oxygen abundances are from J. Moustakas et al. (2010, in preparation).

In the LVL and SINGS samples we retain four galaxies, M81DwA, HolmbergIX, UGC6900, and UGC9128, which are

\footnotetext{
19 The final LVL $\mathrm{H} \alpha$ sample will include 174 galaxies.
} 
undetected or marginally detected in the MIPS bands, but have $\mathrm{H} \alpha$ measurements; these galaxies are metal poor, and consequently, dust poor, thus likely to have most of their star formation light emerge directly at UV/optical wavelengths, unobscured by dust.

The areas used to normalize luminosities and calculate LSDs are derived, for each galaxy, from the area occupied by the ionized gas emission as traced by the $\mathrm{H} \alpha$. For the LVL and SINGS samples, ionized gas emission sizes are measured directly from our images, and are defined as the semimajor axis of the ellipse that includes $2 / 3$ of the total $\mathrm{H} \alpha$ emission flux. Given the uniform nature of the LVL and SINGS imaging strategies, this implies that the sizes obtained for those galaxies are internally consistent. The " $2 / 3$ ionized gas emission" radius typically includes all or most of the high surface brightness emitting regions in a galaxy. This definition for the emitting area is, therefore, especially useful for our analysis: it ensures some level of uniformity even when including galaxies for which the $\mathrm{H} \alpha$ image depths vary from object to object. This is the case for some of the galaxies described in the next samples (see below), where the only $\mathrm{H} \alpha$ images available are from the literature.

The MIPS-GTO sample of starbursts is described in Engelbracht et al. (2008): the galaxies cover a wide range in metal content, from very metal poor starbursts, like IZw18 and SBS0335-052, to metal-rich examples like IC342. Of the 65 (we consider SBS0335-052E and SBS0335-052W a single galaxy in this paper) galaxies listed in Engelbracht et al. (2008), we only keep the 55 that satisfy one of the following two criteria: (1) they are detected in all three MIPS bands (we exclude NGC 1614 and NGC 3256 which are part of the LIRGs sample, see below); or (2) they are detected and measured in either $\mathrm{H} \alpha$ or $\mathrm{P} \alpha$ (Calzetti et al. 2007). The latter criterion ensures inclusion in the sample of metal-poor galaxies, like IZw18, SBS0335-052, UGCA292, and HS0822+3542, which are marginally detected or undetected in MIPS because their dust content is low. Ten of the starbursts in the MIPS-GTO sample have images in the $\mathrm{P} \alpha(\lambda 1.876 \mu \mathrm{m})$ line (Calzetti et al. 2007), which we use to derive SFRs, after extinction correction using the $\mathrm{H} \alpha$ emission, and to measure the size of the line-emitting region when $\mathrm{H} \alpha$ images are not available. For much of our analysis, we give emphasis to starbursts with both infrared broad-band and optical/near-IR narrow-band detections, since our "reference" SFR indicators generally require information on both (see next section). The distances of the 55 starbursts are typically within $100 \mathrm{Mpc}$, except for one case (Tol2138-405, located at $246 \mathrm{Mpc}$ ). Total MIPS flux values, and literature values for distances, oxygen abundances, and $\mathrm{H} \alpha$ fluxes are listed in Engelbracht et al. (2008). We supplement, where possible, $\mathrm{H} \alpha$ measurements from the literature, when not present in Engelbracht et al. (2008), and we end up with line-emission measurements for a total of 45 galaxies.

In order to obtain the emitting areas for the starbursts, we have searched the literature for images of the ionized gas emission (either $\mathrm{H} \alpha$ or $\mathrm{P} \alpha$ ); we were able to retrieve such information for a total of 44 starburst galaxies. For the 34 galaxies with $\mathrm{H} \alpha$ images available through $\mathrm{NED}^{20}$ in FITS format and/or with $\mathrm{P} \alpha$ images, we derive the ionized gas emission size in the same fashion as for the LVL and SINGS galaxies, with the proviso that non-uniform depths from image to image will reflect into larger uncertainties for the sizes (next section). Finally, sizes derived from published hard-copy images only (10 galaxies) have larger

\footnotetext{
20 NED is the NASA/IPAC Extragalactic Database which is operated by the Jet Propulsion Laboratory, California Institute of Technology, under contract with the National Aeronautics and Space Administration.
}

uncertainties still, as discussed in Section 3. Overall, the values for the sizes of the ionized gas emitting region of the starbursts are the least homogeneous in our sample; variations in sizes of up to $30 \%$ may be expected from differences in the depths and characteristics of the published images.

Ionized gas emission sizes for the 160 total LVL (67), SINGS (69, 31 of which are in common with LVL), and starburst galaxies (55) are listed in Table 1, together with other derived quantities relevant to the present analysis.

Our sample of 160 star-forming and starburst galaxies is augmented with 29 LIRGs from Alonso-Herrero et al. (2000, 2001, 2002, 2006), to expand our SFR surface density range at the high end, covering in total almost 5 orders of magnitude with the combined sample. Selection criteria for the sample are presented in Alonso-Herrero et al. (2006), together with measurements of the $\mathrm{P} \alpha$ flux and extinction corrections, as well as information on the physical extent of the star-forming area for 24 LIRGs. For a few of the LIRGs with extended ionized gas emission, size information is taken from Hattori et al. (2004). To the 24 LIRGs, we add five additional LIRGs (NGC 1614, NGC 3256, NGC 3690, NGC 5653, and Zw049.057) with data from Alonso-Herrero et al. (2000, 2001, 2002). Infrared measurements at 25, 60, and $100 \mu \mathrm{m}$ from IRAS and distances (which we rescale to our adopted value of the Hubble constant) for each galaxy are from Sanders et al. (2003) and Surace et al. (2004). For two of the LIRGs, NGC 1614 and NGC 3256, MIPS measurements are available from Engelbracht et al. (2008). Although individual oxygen abundance measurements are not available for the majority of the LIRGs, their metallicities are characteristic of high-metallicity galaxies (Alonso-Herrero et al. 2006). Basic parameters for the 29 LIRGs are listed in Table 2.

The final sample consists of 189 nearby star-forming galaxies, which we divide into a "high-metallicity" sample, consisting of 142 objects with oxygen abundances $12+\log (\mathrm{O} / \mathrm{H}) \gtrsim 8.1(113$ normal star-forming and starburst and 29 LIRGS), and a "lowmetallicity" sample of 47 objects with oxygen abundance 12 $+\log (\mathrm{O} / \mathrm{H})<8.1$ (Table 1$)$. We do not consider the sample large enough to be reasonably divided into more than two metallicity bins. The separating value in metallicity is chosen after Engelbracht et al. (2005), Jackson et al. (2006), Draine et al. (2007), and Engelbracht et al. (2008), where it is found that galaxies roughly below this metallicity value tend to be underluminous in their polycyclic aromatic hydrocarbon (PAH) emission (as measured in the Spitzer-IRAC $8 \mu \mathrm{m}$ band). We do not expect the striking trend of the PAH bands to be also present in the thermal-dust-emission-dominated longer wavelength bands. However, we do expect that, as the metal and dust content of galaxies decreases, the galaxies become increasingly transparent, i.e., their dust opacity decreases, and the infrared dust emission will be a progressively less accurate tracer of the current SFR. We thus select the value $12+\log (\mathrm{O} / \mathrm{H})$ $\sim 8.1$ as a convenient "transition" value for dust content in our galaxies. In Section 7, we also discuss the implications of further dividing the high-metallicity sample into two subsamples, in order to investigate the role of metal abundance in driving some of the observed scatter in the data.

The range of infrared luminosity covered by our sample is shown in Figure 1, together with the range of 70-160 $\mu \mathrm{m}$ luminosity ratio, the latter being a rough proxy for effective dust temperature (e.g., Engelbracht et al. 2008). The combination of low-metallicity and high-metallicity star-forming and starburst galaxies plus the addition of the LIRGs enables us to explore almost 6 orders of magnitude in to- 
Table 1

Properties of Normal Star-Forming and Starburst Galaxies

\begin{tabular}{|c|c|c|c|c|c|c|c|c|c|c|}
\hline Name $^{\mathrm{a}}$ & $\begin{array}{c}D^{\mathrm{b}} \\
(\mathrm{Mpc})\end{array}$ & $12+\log (\mathrm{O} / \mathrm{H})^{\mathrm{c}}$ & $\begin{array}{l}R_{25^{\mathrm{d}}} \\
\left({ }^{\prime \prime}\right) \\
\end{array}$ & $\begin{array}{c}R_{\mathrm{H} \alpha} \mathrm{e} \\
(")\end{array}$ & Ref. $^{\text {e }}$ & $\begin{array}{c}\log \left(\Sigma_{\mathrm{SFR}}\right)^{\mathrm{f}} \\
\left(M_{\odot} \mathrm{yr}^{-1} \mathrm{kpc}^{-2}\right) \\
\end{array}$ & $\begin{array}{c}\log \left(\Sigma_{70}\right)^{\mathrm{g}} \\
\left(\mathrm{erg} \mathrm{s}^{-1} \mathrm{kpc}^{-2}\right) \\
\end{array}$ & $\begin{array}{c}\log \left(\Sigma_{160}\right)^{\mathrm{g}} \\
\left(\mathrm{erg} \mathrm{s}^{-1} \mathrm{kpc}^{-2}\right)\end{array}$ & $\begin{array}{c}\log \left(\Sigma_{\mathrm{TIR}}\right)^{\mathrm{g}} \\
\left(\mathrm{erg} \mathrm{s}^{-1} \mathrm{kpc}^{-2}\right)\end{array}$ & Ref. $^{\text {h }}$ \\
\hline \multicolumn{11}{|c|}{ High-metallicity galaxies } \\
\hline NGC 0625 & 4.07 & 8.1 & 172.4 & 60.2 & $\mathrm{~L}$ & $-1.838 \pm 0.101$ & $41.10 \pm 0.09$ & $40.85 \pm 0.10$ & $41.43 \pm 0.11$ & K08,D09 \\
\hline NGC 1522 & 9.99 & 8.2 & 36.05 & 13.5 & $\mathrm{~L}$ & $-1.435 \pm 0.101$ & $41.56 \pm 0.09$ & $41.13 \pm 0.10$ & $41.80 \pm 0.11$ & K08,D09 \\
\hline NGC 1800 & 8.83 & 8.36 & 59.85 & 19.6 & $\mathrm{~L}$ & $-1.713 \pm 0.101$ & $41.34 \pm 0.09$ & $41.17 \pm 0.10$ & $41.62 \pm 0.11$ & K08,D09 \\
\hline UGC4278 & 8.13 & 8.08 & 140.3 & 144.8 & $\mathrm{~L}$ & $-3.407 \pm 0.101$ & $39.47 \pm 0.09$ & $39.36 \pm 0.10$ & $39.77 \pm 0.11$ & K08,D09 \\
\hline UGC5829 & 8.44 & 8.3 & 140.3 & 140.8 & $\mathrm{~L}$ & $-3.404 \pm 0.101$ & $39.40 \pm 0.09$ & $39.14 \pm 0.10$ & $39.63 \pm 0.11$ & K08,D09 \\
\hline NGC 3368 & 11.27 & 9.04 & 227.6 & 84.8 & $\mathrm{~L}$ & $-2.274 \pm 0.101$ & $41.13 \pm 0.09$ & $41.30 \pm 0.10$ & $41.61 \pm 0.11$ & K08,D09 \\
\hline UGC5923 & 7.67 & 8.26 & 28 & 19.6 & $\mathrm{~L}$ & $-2.501 \pm 0.101$ & $40.65 \pm 0.09$ & $40.27 \pm 0.10$ & $40.83 \pm 0.12$ & K08,D09 \\
\hline NGC 3510 & 9.18 & 8.08 & 119.5 & 99.6 & $\mathrm{~L}$ & $-3.123 \pm 0.101$ & $39.92 \pm 0.09$ & $39.75 \pm 0.10$ & $40.19 \pm 0.11$ & K08,D09 \\
\hline NGC 3623 & 9.59 & 9.06 & 293.1 & 125.2 & $\mathrm{~L}$ & $-2.693 \pm 0.101$ & $40.49 \pm 0.09$ & $40.84 \pm 0.10$ & $41.11 \pm 0.11$ & K08,D09 \\
\hline UGC6900 & 8 & 8.1 & 62.7 & 27.8 & $\mathrm{~L}$ & $-3.107 \pm 0.103$ & $39.74 \pm 0.30$ & $39.96 \pm 0.30$ & $40.26 \pm 0.38$ & K08,D09 \\
\hline NGC 4248 & 7.76 & 8.15 & 90.6 & 27.4 & $\mathrm{~L}$ & $-2.508 \pm 0.101$ & $40.66 \pm 0.09$ & $40.71 \pm 0.10$ & $41.08 \pm 0.11$ & K08,D09 \\
\hline NGC 4288 & 8.22 & 8.52 & 64.15 & 48.2 & $\mathrm{~L}$ & $-2.486 \pm 0.101$ & $40.61 \pm 0.09$ & $40.55 \pm 0.10$ & $40.95 \pm 0.11$ & K08,D09 \\
\hline UGC7490 & 9 & 8.46 & 99.35 & 83.5 & $\mathrm{~L}$ & $-3.325 \pm 0.101$ & $39.59 \pm 0.09$ & $39.78 \pm 0.10$ & $40.08 \pm 0.11$ & K08,D09 \\
\hline UGC7699 & 7.34 & 8.15 & 114.1 & 89.6 & $\mathrm{~L}$ & $-3.204 \pm 0.101$ & $39.80 \pm 0.09$ & $39.77 \pm 0.10$ & $40.15 \pm 0.11$ & K08,D09 \\
\hline UGC7698 & 6.1 & 8.04 & 193.7 & 145.2 & $\mathrm{~L}$ & $-3.620 \pm 0.101$ & $38.78 \pm 0.09$ & $38.77 \pm 0.10$ & $39.14 \pm 0.12$ & K08,D09 \\
\hline NGC 4656 & 9.2 & 8.78 & 454 & 282.6 & $\mathrm{~L}$ & $-2.979 \pm 0.101$ & $39.90 \pm 0.09$ & $39.67 \pm 0.10$ & $40.17 \pm 0.11$ & K08,D09 \\
\hline UGC7950 & 8.48 & 8.37 & 38.65 & 40.4 & $\mathrm{~L}$ & $-3.024 \pm 0.101$ & $40.11 \pm 0.09$ & $39.89 \pm 0.10$ & $40.35 \pm 0.12$ & K08,D09 \\
\hline NGC 4707 & 7.97 & 8.43 & 67.15 & 67.2 & $\mathrm{~L}$ & $-3.219 \pm 0.101$ & $39.54 \pm 0.09$ & $39.50 \pm 0.10$ & $39.89 \pm 0.12$ & K08,D09 \\
\hline UGCA320 & 7.76 & 8.08 & 168.7 & 155.2 & $\mathrm{~L}$ & $-3.313 \pm 0.101$ & $39.17 \pm 0.09$ & $38.79 \pm 0.10$ & $39.36 \pm 0.12$ & K08,D09 \\
\hline UGC8320 & 4.33 & 8.29 & 108.9 & 95.2 & $\mathrm{~L}$ & $-3.367 \pm 0.101$ & $39.64 \pm 0.09$ & $39.42 \pm 0.10$ & $39.87 \pm 0.12$ & K08,D09 \\
\hline NGC 5068 & 6.24 & 8.82 & 217.4 & 206.7 & $\mathrm{~L}$ & $-2.438 \pm 0.101$ & $40.53 \pm 0.09$ & $40.59 \pm 0.10$ & $40.94 \pm 0.11$ & K08,D09 \\
\hline NGC 5477 & 8.25 & 8.14 & 49.8 & 55.6 & $\mathrm{~L}$ & $-2.798 \pm 0.101$ & $39.98 \pm 0.09$ & $39.63 \pm 0.10$ & $40.17 \pm 0.12$ & K08,D09 \\
\hline NGC 0024 & 8.13 & 8.62 & 172.7 & 143. & $\mathrm{~L}, \mathrm{~S}$ & $-3.075 \pm 0.101$ & $39.91 \pm 0.09$ & $40.09 \pm 0.10$ & $40.39 \pm 0.11$ & K09,D07 \\
\hline NGC 0337 & 24.69 & 8.56 & 86.5 & 109. & $\mathrm{~S}$ & $-2.399 \pm 0.101$ & $40.81 \pm 0.09$ & $40.71 \pm 0.10$ & $41.14 \pm 0.11$ & K09,D07 \\
\hline NGC 0628 & 7.32 & 8.67 & 314.1 & 261. & $\mathrm{~L}, \mathrm{~S}$ & $-2.583 \pm 0.101$ & $40.55 \pm 0.09$ & $40.75 \pm 0.10$ & $41.07 \pm 0.11$ & K09,D07 \\
\hline NGC 0925 & 9.12 & 8.51 & 314.1 & 296. & $\mathrm{~S}$ & $-2.995 \pm 0.101$ & $40.06 \pm 0.09$ & $40.18 \pm 0.10$ & $40.51 \pm 0.11$ & K09,D07 \\
\hline NGC 1097 & 16.88 & 8.78 & 280 & 282. & $\mathrm{~S}$ & $-2.453 \pm 0.101$ & $40.72 \pm 0.09$ & $40.77 \pm 0.10$ & $41.16 \pm 0.11$ & K09,D07 \\
\hline NGC 1291 & 9.83 & 8.72 & 293.1 & 329. & $\mathrm{~L}, \mathrm{~S}$ & $-3.392 \pm 0.101$ & $39.61 \pm 0.09$ & $39.90 \pm 0.10$ & $40.19 \pm 0.11$ & K09,D07 \\
\hline NGC 1377 & 24.17 & 8.37 & 53.35 & 44.3 & S & $-1.674 \pm 0.101$ & $41.36 \pm 0.09$ & $40.73 \pm 0.10$ & $41.74 \pm 0.11$ & K09,D07 \\
\hline NGC 1482 & 22.03 & 8.53 & 73.65 & 59.1 & $\mathrm{~S}$ & $-1.567 \pm 0.101$ & $41.81 \pm 0.09$ & $41.53 \pm 0.10$ & $42.11 \pm 0.11$ & K09,D07 \\
\hline NGC 1512 & 10.14 & 8.71 & 267.4 & 185. & $\mathrm{~L}, \mathrm{~S}$ & $-2.977 \pm 0.101$ & $40.13 \pm 0.09$ & $40.32 \pm 0.10$ & $40.63 \pm 0.11$ & K09,D07 \\
\hline NGC 1566 & 18.27 & 8.82 & 249.6 & 269. & $\mathrm{~S}$ & $-2.704 \pm 0.101$ & $40.52 \pm 0.09$ & $40.63 \pm 0.10$ & $40.98 \pm 0.11$ & K09,D07 \\
\hline NGC 1705 & 5.1 & 8.32 & 57.15 & 57.2 & $\mathrm{~L}, \mathrm{~S}$ & $-2.043 \pm 0.101$ & $40.47 \pm 0.09$ & $40.19 \pm 0.10$ & $40.69 \pm 0.11$ & K09,D07 \\
\hline NGC 2403 & 3.22 & 8.56 & 656.3 & 413. & $\mathrm{~L}, \mathrm{~S}$ & $-2.492 \pm 0.101$ & $40.55 \pm 0.09$ & $40.64 \pm 0.10$ & $40.99 \pm 0.11$ & K09,D07 \\
\hline NGC 2798 & 24.68 & 8.69 & 77.1 & 26.0 & $\mathrm{~S}$ & $-0.992 \pm 0.101$ & $42.35 \pm 0.09$ & $41.97 \pm 0.10$ & $42.62 \pm 0.11$ & K09,D07 \\
\hline NGC 2841 & 9.81 & 8.86 & 243.9 & 209. & $\mathrm{~S}$ & $-2.418 \pm 0.101$ & $40.21 \pm 0.09$ & $40.64 \pm 0.10$ & $40.89 \pm 0.11$ & K09,D07 \\
\hline NGC 2915 & 3.78 & 8.16 & 57.15 & 51.4 & G03 & $-2.152 \pm 0.101$ & $40.57 \pm 0.09$ & $40.23 \pm 0.10$ & $40.77 \pm 0.11$ & G03,D07 \\
\hline NGC 2976 & 3.56 & 8.64 & 176.7 & 117. & $\mathrm{~L}, \mathrm{~S}$ & $-2.189 \pm 0.101$ & $41.02 \pm 0.09$ & $41.07 \pm 0.10$ & $41.43 \pm 0.11$ & K09,D07 \\
\hline NGC 3049 & 19.1 & 8.75 & 65.65 & 81.3 & $\mathrm{~S}$ & $-2.471 \pm 0.101$ & $40.49 \pm 0.09$ & $40.35 \pm 0.10$ & $40.87 \pm 0.11$ & K09,D07 \\
\hline NGC 3031 & 3.63 & 8.69 & 692.7 & 680. & $\mathrm{~L}, \mathrm{~S}$ & $-3.020 \pm 0.101$ & $40.11 \pm 0.09$ & $40.37 \pm 0.10$ & $40.65 \pm 0.11$ & P06,D07 \\
\hline NGC 3034 & 3.89 & 8.72 & 336.6 & 143. & $\mathrm{~L}, \mathrm{~S}$ & $-0.463 \pm 0.101$ & $42.74 \pm 0.09$ & $41.98 \pm 0.10$ & $43.00 \pm 0.11$ & K09,D07 \\
\hline NGC 3190 & 16.81 & 8.7 & 130.9 & 103. & $\mathrm{~S}$ & $-2.882 \pm 0.101$ & $40.57 \pm 0.09$ & $40.63 \pm 0.10$ & $40.97 \pm 0.11$ & K09,D07 \\
\hline NGC 3184 & 8.53 & 8.81 & 222.4 & 217. & S & $-2.859 \pm 0.101$ & $40.37 \pm 0.09$ & $40.66 \pm 0.10$ & $40.95 \pm 0.11$ & K09,D07 \\
\hline NGC 3198 & 13.68 & 8.6 & 255.4 & 233. & S & $-2.897 \pm 0.101$ & $40.12 \pm 0.09$ & $40.34 \pm 0.10$ & $40.66 \pm 0.11$ & K09,D07 \\
\hline NGC 3265 & 19.49 & 8.65 & 38.65 & 44.3 & $\mathrm{~S}$ & $-2.162 \pm 0.101$ & $40.99 \pm 0.09$ & $40.63 \pm 0.10$ & $41.26 \pm 0.11$ & K09,D07 \\
\hline Mrk33 & 21.92 & 8.56 & 30. & 26.1 & $\mathrm{~S}$ & $-1.276 \pm 0.101$ & $41.65 \pm 0.09$ & $41.24 \pm 0.10$ & $41.99 \pm 0.11$ & K09,D07 \\
\hline NGC 3351 & 9.33 & 8.91 & 222.4 & 189. & $\mathrm{~L}, \mathrm{~S}$ & $-2.597 \pm 0.101$ & $40.67 \pm 0.09$ & $40.76 \pm 0.10$ & $41.13 \pm 0.11$ & K08,D07 \\
\hline NGC 3521 & 8.99 & 8.68 & 328.9 & 284. & $\mathrm{~L}, \mathrm{~S}$ & $-2.583 \pm 0.101$ & $40.74 \pm 0.09$ & $40.92 \pm 0.10$ & $41.25 \pm 0.11$ & K08,D07 \\
\hline NGC 3621 & 6.64 & 8.5 & 369.1 & 531. & $\mathrm{~S}$ & $-3.246 \pm 0.101$ & $40.09 \pm 0.09$ & $40.18 \pm 0.10$ & $40.53 \pm 0.11$ & K09,D07 \\
\hline NGC 3627 & 9.38 & 8.8 & 273.6 & 244. & $\mathrm{~L}, \mathrm{~S}$ & $-2.358 \pm 0.101$ & $41.03 \pm 0.09$ & $41.07 \pm 0.10$ & $41.45 \pm 0.11$ & K09,D07 \\
\hline NGC 3773 & 12.53 & 8.64 & 35.25 & 35.1 & $\mathrm{~S}$ & $-1.941 \pm 0.101$ & $40.95 \pm 0.09$ & $40.77 \pm 0.10$ & $41.27 \pm 0.11$ & K09,D07 \\
\hline NGC 3938 & 12.22 & 8.71 & 161.1 & 191. & S & $-2.687 \pm 0.101$ & $40.43 \pm 0.09$ & $40.64 \pm 0.10$ & $40.95 \pm 0.11$ & K09,D07 \\
\hline NGC 4125 & 22.91 & 8.89 & 172.7 & 95. & $\mathrm{~S}$ & $-2.848 \pm 0.101$ & $39.93 \pm 0.09$ & $39.77 \pm 0.10$ & $40.24 \pm 0.11$ & K09,D07 \\
\hline NGC 4236 & 4.45 & 8.31 & 656.3 & 582. & $\mathrm{~L}, \mathrm{~S}$ & $-3.567 \pm 0.101$ & $39.23 \pm 0.09$ & $39.26 \pm 0.10$ & $39.63 \pm 0.11$ & K08,D07 \\
\hline NGC 4254 & 33.29 & 8.8 & 161.1 & 185. & $\mathrm{~S}$ & $-2.126 \pm 0.101$ & $41.01 \pm 0.09$ & $41.10 \pm 0.10$ & $41.46 \pm 0.11$ & K09,D07 \\
\hline NGC 4321 & 14.32 & 8.84 & 222.4 & 195. & $\mathrm{~S}$ & $-2.343 \pm 0.101$ & $40.87 \pm 0.09$ & $41.05 \pm 0.10$ & $41.37 \pm 0.11$ & K09,D07 \\
\hline NGC 4450 & 27.07 & 8.85 & 157.4 & 113. & $\mathrm{~S}$ & $-3.019 \pm 0.101$ & $40.27 \pm 0.09$ & $40.61 \pm 0.10$ & $40.87 \pm 0.11$ & K09,D07 \\
\hline NGC 4536 & 14.45 & 8.6 & 227.6 & 229. & $\mathrm{~S}$ & $-2.574 \pm 0.101$ & $40.63 \pm 0.09$ & $40.53 \pm 0.10$ & $40.99 \pm 0.11$ & K09,D07 \\
\hline NGC 4559 & 11.57 & 8.51 & 321.4 & 267. & $\mathrm{~S}$ & $-2.845 \pm 0.101$ & $40.22 \pm 0.09$ & $40.36 \pm 0.10$ & $40.69 \pm 0.11$ & K09,D07 \\
\hline NGC 4569 & 16 & 8.88 & 286.5 & 270. & K01 & $-3.254 \pm 0.101$ & $40.07 \pm 0.09$ & $40.24 \pm 0.10$ & $40.58 \pm 0.11$ & B02,D07 \\
\hline NGC 4579 & 20.62 & 8.93 & 176.7 & 116. & $\mathrm{~S}$ & $-2.387 \pm 0.101$ & $40.69 \pm 0.09$ & $40.97 \pm 0.10$ & $41.26 \pm 0.11$ & K09,D07 \\
\hline NGC 4594 & 9.33 & 8.99 & 261.3 & 201. & $\mathrm{~L}, \mathrm{~S}$ & $-3.138 \pm 0.101$ & $40.14 \pm 0.09$ & $40.50 \pm 0.10$ & $40.77 \pm 0.11$ & K09,D07 \\
\hline NGC 4625 & 9.51 & 8.65 & 65.65 & 38.0 & $\mathrm{~L}, \mathrm{~S}$ & $-2.245 \pm 0.101$ & $41.00 \pm 0.09$ & $41.06 \pm 0.10$ & $41.41 \pm 0.11$ & K09,D07 \\
\hline NGC 4631 & 8.92 & 8.44 & 464.6 & 257. & $\mathrm{~L}, \mathrm{~S}$ & $-2.222 \pm 0.101$ & $41.14 \pm 0.09$ & $41.12 \pm 0.10$ & $41.51 \pm 0.11$ & K09,D07 \\
\hline
\end{tabular}


Table 1

(Continued)

\begin{tabular}{|c|c|c|c|c|c|c|c|c|c|c|}
\hline Name $^{\mathrm{a}}$ & $\begin{array}{c}D^{\mathrm{b}} \\
(\mathrm{Mpc}) \\
\end{array}$ & $12+\log (\mathrm{O} / \mathrm{H})^{\mathrm{c}}$ & $\begin{array}{l}R_{25}{ }^{\mathrm{d}} \\
\left({ }^{\prime \prime}\right)\end{array}$ & $\begin{array}{c}R_{\mathrm{H} \alpha}{ }^{\mathrm{e}} \\
\left({ }^{\prime \prime}\right)\end{array}$ & Ref. $^{\text {e }}$ & $\begin{array}{c}\log \left(\Sigma_{\mathrm{SFR}}\right)^{\mathrm{f}} \\
\left(M_{\odot} \mathrm{yr}^{-1} \mathrm{kpc}^{-2}\right)\end{array}$ & $\begin{array}{c}\log \left(\Sigma_{70}\right)^{\mathrm{g}} \\
\left(\mathrm{erg} \mathrm{s}^{-1} \mathrm{kpc}^{-2}\right)\end{array}$ & $\begin{array}{c}\log \left(\Sigma_{160}\right)^{\mathrm{g}} \\
\left(\mathrm{erg} \mathrm{s}^{-1} \mathrm{kpc}^{-2}\right) \\
\end{array}$ & $\begin{array}{c}\log \left(\Sigma_{\mathrm{TIR}}\right)^{\mathrm{g}} \\
\left(\mathrm{erg} \mathrm{s}^{-1} \mathrm{kpc}^{-2}\right) \\
\end{array}$ & Ref. $^{\text {h }}$ \\
\hline NGC 4725 & 17.12 & 8.73 & 321.4 & 240 & $\mathrm{~S}$ & $-3.132 \pm 0.101$ & $40.03 \pm 0.09$ & $40.50 \pm 0.10$ & $40.74 \pm 0.11$ & K09,D07 \\
\hline NGC 4736 & 5.01 & 8.66 & 336.6 & 238. & $\mathrm{~L}, \mathrm{~S}$ & $-2.380 \pm 0.101$ & $41.06 \pm 0.09$ & $40.98 \pm 0.10$ & $41.39 \pm 0.11$ & K08,D07 \\
\hline NGC 4826 & 5.54 & 8.92 & 300 & 84. & $\mathrm{~L}, \mathrm{~S}$ & $-1.753 \pm 0.101$ & $41.73 \pm 0.09$ & $41.63 \pm 0.10$ & $42.05 \pm 0.11$ & K09,D07 \\
\hline NGC 5055 & 8.32 & 8.78 & 377.7 & 226. & $\mathrm{~L}, \mathrm{~S}$ & $-2.361 \pm 0.101$ & $40.99 \pm 0.09$ & $41.25 \pm 0.10$ & $41.55 \pm 0.11$ & K09,D07 \\
\hline NGC 5194 & 8.13 & 8.86 & 336.6 & 296. & $\mathrm{~L}, \mathrm{~S}$ & $-2.244 \pm 0.101$ & $41.07 \pm 0.09$ & $41.23 \pm 0.10$ & $41.56 \pm 0.11$ & K09,D07 \\
\hline Tol89 & 14.9 & 8.54 & 84.55 & 81. & S & $-2.572 \pm 0.101$ & $40.33 \pm 0.09$ & $40.21 \pm 0.10$ & $40.72 \pm 0.11$ & K09,D07 \\
\hline NGC 5474 & 6.8 & 8.32 & 143.6 & 131. & $\mathrm{~L}, \mathrm{~S}$ & $-2.810 \pm 0.101$ & $40.18 \pm 0.09$ & $40.27 \pm 0.10$ & $40.60 \pm 0.11$ & K09,D07 \\
\hline NGC 5713 & 26.49 & 8.64 & 82.65 & 52.2 & $\mathrm{~S}$ & $-1.563 \pm 0.101$ & $41.78 \pm 0.09$ & $41.65 \pm 0.10$ & $42.13 \pm 0.11$ & K09,D07 \\
\hline NGC 5866 & 15.14 & 8.72 & 140.3 & 100. & $\mathrm{~S}$ & $-2.830 \pm 0.101$ & $40.78 \pm 0.09$ & $40.73 \pm 0.10$ & $41.10 \pm 0.11$ & K09,D07 \\
\hline IC4710 & 8.48 & 8.37 & 108.9 & 109. & S & $-2.823 \pm 0.101$ & $40.14 \pm 0.09$ & $39.96 \pm 0.10$ & $40.42 \pm 0.11$ & K09,D07 \\
\hline NGC 6822 & 0.47 & 8.35 & 464.6 & 765. & S & $-3.133 \pm 0.101$ & $39.88 \pm 0.09$ & $39.87 \pm 0.10$ & $40.24 \pm 0.11$ & K09,D07 \\
\hline NGC 6946 & 6.8 & 8.72 & 344.4 & 217. & S & $-1.755 \pm 0.101$ & $41.49 \pm 0.09$ & $41.51 \pm 0.10$ & $41.91 \pm 0.11$ & K09,D07 \\
\hline NGC 7331 & 14.52 & 8.7 & 314.1 & 295. & S & $-2.693 \pm 0.101$ & $40.78 \pm 0.09$ & $40.82 \pm 0.10$ & $41.18 \pm 0.11$ & K09,D07 \\
\hline NGC 7552 & 22.27 & 8.74 & 101.7 & 113. & $\mathrm{~S}$ & $-1.657 \pm 0.101$ & $41.57 \pm 0.09$ & $41.35 \pm 0.10$ & $41.93 \pm 0.11$ & K09,D07 \\
\hline NGC 7793 & 3.82 & 8.53 & 255 & 286. & $\mathrm{~L}, \mathrm{~S}$ & $-2.549 \pm 0.101$ & $40.46 \pm 0.09$ & $40.67 \pm 0.10$ & $40.97 \pm 0.11$ & K09,D07 \\
\hline Mrk170 & 20.4 & 8.09 & 31.4 & 18.1 & A & $>-2.877 \pm 0.153$ & $40.91 \pm 0.15$ & $40.62 \pm 0.15$ & $41.14 \pm 0.15$ & . .,E08 \\
\hline Mrk930 & 77 & 8.11 & 25.8 & 14.9 & $\mathrm{~A}$ & $>-1.748 \pm 0.153$ & $41.53 \pm 0.15$ & $40.90 \pm 0.15$ & $41.79 \pm 0.15$ & ...,E08 \\
\hline NGC 1569 & 1.9 & 8.13 & 108.9 & 85.9 & $\mathrm{H} 04$ & $-0.890 \pm 0.153$ & $41.58 \pm 0.15$ & $41.20 \pm 0.15$ & $41.93 \pm 0.15$ & H04,E08 \\
\hline Mrk1094 & 41 & 8.15 & 20.75 & 19.8 & G03 & $-1.540 \pm 0.153$ & $41.21 \pm 0.15$ & $40.76 \pm 0.15$ & $41.40 \pm 0.15$ & G03,E08 \\
\hline NGC 3310 & 21.3 & 8.18 & 92.7 & 67.2 & J04 & $-1.263 \pm 0.153$ & $41.67 \pm 0.15$ & $41.29 \pm 0.15$ & $41.98 \pm 0.15$ & J04,E08 \\
\hline Mrk162 & 98 & 8.19 & 18.1 & 10.5 & $\mathrm{~A}$ & $>-1.474 \pm 0.153$ & $41.94 \pm 0.15$ & $41.61 \pm 0.15$ & $42.23 \pm 0.15$ & $\ldots$, . E08 \\
\hline NGC 1156 & 7.8 & 8.19 & 99.35 & 88.0 & $\mathrm{H} 04$ & $-2.159 \pm 0.153$ & $40.76 \pm 0.15$ & $40.13 \pm 0.15$ & $40.94 \pm 0.15$ & H04,E08 \\
\hline Tol2 & 22 & 8.22 & 25 & 19.4 & G03 & $-1.506 \pm 0.153$ & $41.21 \pm 0.15$ & $40.62 \pm 0.15$ & $41.38 \pm 0.15$ & G03,E08 \\
\hline MinkObj & 78 & 8.22 & 15 & 13.9 & $\mathrm{C} 06$ & $-2.364 \pm 0.153$ & $40.13 \pm 0.15$ & $39.62 \pm 0.31$ & $40.34 \pm 0.32$ & C06,E08 \\
\hline NGC 4449 & 4.2 & 8.23 & 185 & 133.5 & $\mathrm{~L}$ & $-1.819 \pm 0.153$ & $41.17 \pm 0.15$ & $41.10 \pm 0.15$ & $41.53 \pm 0.15$ & K08,E08 \\
\hline NGC 7714 & 40 & 8.26 & 57.15 & 24.2 & J04 & $-0.873 \pm 0.153$ & $42.04 \pm 0.15$ & $41.58 \pm 0.15$ & $42.41 \pm 0.15$ & J04,E08 \\
\hline UGC4703 & 57 & 8.31 & 13.7 & 6.0 & M99 & $-1.068 \pm 0.153$ & $41.61 \pm 0.15$ & $41.43 \pm 0.15$ & $41.96 \pm 0.16$ & M99,E08 \\
\hline NGC 1140 & 21.2 & 8.32 & 49.8 & 44.0 & H94 & $-1.881 \pm 0.153$ & $41.06 \pm 0.15$ & $40.79 \pm 0.15$ & $41.37 \pm 0.15$ & H94,E08 \\
\hline NGC 1510 & 11.8 & 8.33 & 39.55 & 10.9 & $\mathrm{~S}$ & $-1.114 \pm 0.153$ & $41.81 \pm 0.15$ & $41.36 \pm 0.15$ & $42.06 \pm 0.15$ & K09,E08 \\
\hline NGC 3125 & 12 & 8.34 & 32.9 & 22.9 & G03 & $-1.083 \pm 0.153$ & $41.84 \pm 0.15$ & $41.40 \pm 0.15$ & $42.11 \pm 0.15$ & G03,E08 \\
\hline NGC 4214 & 2.9 & 8.36 & 255.4 & 188.6 & $\mathrm{H} 04$ & $-2.310 \pm 0.153$ & $40.57 \pm 0.15$ & $40.29 \pm 0.15$ & $40.86 \pm 0.15$ & K08,E08 \\
\hline NGC 4670 & 23.2 & 8.38 & 42.4 & 28.1 & G03 & $-1.539 \pm 0.153$ & $41.46 \pm 0.15$ & $41.18 \pm 0.15$ & $41.73 \pm 0.15$ & G03,E08 \\
\hline $\mathrm{He} 2-10$ & 9 & 8.55 & 52.15 & 20.7 & $\mathrm{~J} 00$ & $-0.468 \pm 0.153$ & $42.47 \pm 0.15$ & $42.03 \pm 0.15$ & $42.89 \pm 0.15$ & J00,E08 \\
\hline NGC 3628 & 13.1 & 8.57 & 443.8 & 240. & F90 & $-2.530 \pm 0.153$ & $40.94 \pm 0.15$ & $40.88 \pm 0.15$ & $41.31 \pm 0.15$ & K08,E08 \\
\hline NGC 3079 & 21.8 & 8.57 & 238.3 & 180. & $\mathrm{R} 07$ & $-2.551 \pm 0.153$ & $41.11 \pm 0.15$ & $40.92 \pm 0.15$ & $41.38 \pm 0.15$ & L96,E08 \\
\hline NGC 2782 & 42 & 8.59 & 104 & 30. & E96 & $-1.354 \pm 0.153$ & $41.87 \pm 0.15$ & $41.58 \pm 0.15$ & $42.17 \pm 0.15$ & M06,E08 \\
\hline NGC 3077 & 3.8 & 8.6 & 161.1 & 27.6 & J04 & $-0.944 \pm 0.153$ & $42.24 \pm 0.15$ & $41.83 \pm 0.15$ & $42.47 \pm 0.15$ & K08,E08 \\
\hline NGC 3367 & 49 & 8.62 & 75.35 & 50.3 & G01 & $-1.744 \pm 0.153$ & $41.30 \pm 0.15$ & $41.21 \pm 0.15$ & $41.72 \pm 0.15$ & M06,E08 \\
\hline NGC 5236 & 4.5 & 8.62 & 386.4 & 305. & L99 & $-1.774 \pm 0.153$ & $41.34 \pm 0.15$ & $41.43 \pm 0.15$ & $41.82 \pm 0.15$ & K08,E08 \\
\hline NGC 5953 & 35 & 8.67 & 48.65 & 17. & $\mathrm{H} 03$ & $-1.037 \pm 0.153$ & $42.32 \pm 0.15$ & $42.20 \pm 0.15$ & $42.66 \pm 0.15$ & K87,E08 \\
\hline NGC 4194 & 42 & 8.67 & 54.6 & 13.5 & HT04 & $-0.242 \pm 0.153$ & $42.84 \pm 0.15$ & $42.33 \pm 0.15$ & $43.17 \pm 0.15$ & HT04,E08 \\
\hline NGC 2903 & 8.9 & 8.68 & 377.7 & 146. & B02 & $-1.814 \pm 0.153$ & $41.37 \pm 0.15$ & $41.33 \pm 0.15$ & $41.78 \pm 0.15$ & K08,E08 \\
\hline Mrk25 & 48 & 8.68 & 16.5 & 9.5 & A & $>-1.287 \pm 0.153$ & $41.99 \pm 0.15$ & $41.52 \pm 0.15$ & $42.29 \pm 0.15$ & . .,E08 \\
\hline Mrk331 & 78 & 8.76 & 20.3 & 10. & HT04 & $-0.368 \pm 0.153$ & $42.99 \pm 0.15$ & $42.59 \pm 0.15$ & $43.27 \pm 0.15$ & HT04,E08 \\
\hline IC342 & 3.3 & 8.85 & 641.4 & 380. & H05 & $-1.787 \pm 0.153$ & $41.26 \pm 0.15$ & $41.25 \pm 0.15$ & $41.67 \pm 0.15$ & W99,E08 \\
\hline IIZw40 & 9.2 & 8.11 & 12.5 & 10.4 & G03 & $-0.015 \pm 0.108$ & $42.54 \pm 0.15$ & $41.95 \pm 0.15$ & $42.94 \pm 0.15$ & C07,E08 \\
\hline NGC 5253 & 4 & 8.19 & 150. & 22.2 & C99 & $0.017 \pm 0.108$ & $42.95 \pm 0.15$ & $42.43 \pm 0.15$ & $43.39 \pm 0.15$ & C07,E08 \\
\hline NGC 2537 & 6.9 & 8.44 & 52.15 & 28.4 & J04 & $-1.506 \pm 0.108$ & $41.53 \pm 0.15$ & $41.34 \pm 0.15$ & $41.83 \pm 0.15$ & C07,E08 \\
\hline NGC 2146 & 17.9 & 8.68 & 180.8 & 35.5 & J04 & $-0.540 \pm 0.108$ & $42.89 \pm 0.15$ & $42.43 \pm 0.15$ & $43.15 \pm 0.15$ & $\mathrm{C} 07, \mathrm{E} 08$ \\
\hline \multicolumn{11}{|c|}{ Low-metallicity galaxies } \\
\hline WLM & 0.92 & 7.77 & 344.4 & 107.9 & $\mathrm{~L}$ & $-2.751 \pm 0.101$ & $40.08 \pm 0.09$ & $40.00 \pm 0.10$ & $40.40 \pm 0.11$ & K08,D09 \\
\hline UGC5272 & 7.61 & 7.83 & 62.7 & 62.6 & $\mathrm{~L}$ & $-2.991 \pm 0.101$ & $39.74 \pm 0.09$ & $39.38 \pm 0.10$ & $39.93 \pm 0.12$ & K08,D09 \\
\hline NGC 3109 & 1.34 & 7.77 & 571.6 & 286.7 & $\mathrm{~L}$ & $-3.020 \pm 0.101$ & $39.76 \pm 0.09$ & $39.65 \pm 0.10$ & $40.06 \pm 0.11$ & K08,D09 \\
\hline SextansA & 1.32 & 7.54 & 176.7 & 144.3 & $\mathrm{~L}$ & $-3.015 \pm 0.101$ & $39.37 \pm 0.09$ & $39.15 \pm 0.10$ & $39.62 \pm 0.12$ & K08,D09 \\
\hline UGC5764 & 7.59 & 7.95 & 59.85 & 45.21 & $\mathrm{~L}$ & $-3.285 \pm 0.102$ & $39.31 \pm 0.10$ & $39.11 \pm 0.11$ & $39.59 \pm 0.12$ & K08,D09 \\
\hline UGCA281 & 5.7 & 7.8 & 24.95 & 26.08 & $\mathrm{~L}$ & $-1.739 \pm 0.101$ & $40.66 \pm 0.09$ & $39.89 \pm 0.10$ & $40.87 \pm 0.12$ & K08,D09 \\
\hline UGC8508 & 2.69 & 7.89 & 50.95 & 56.8 & $\mathrm{~L}$ & $-3.141 \pm 0.101$ & $39.50 \pm 0.09$ & $39.26 \pm 0.10$ & $39.74 \pm 0.12$ & K08,D09 \\
\hline UGC8651 & 3.02 & 7.85 & 70.35 & 83.03 & $\mathrm{~L}$ & $-3.500 \pm 0.102$ & $38.87 \pm 0.10$ & $38.76 \pm 0.11$ & $39.18 \pm 0.13$ & K08,D09 \\
\hline UGC8837 & 8.89 & 7.7 & 128 & 93.46 & $\mathrm{~L}$ & $-3.308 \pm 0.101$ & $39.36 \pm 0.09$ & $39.43 \pm 0.10$ & $39.78 \pm 0.12$ & K08,D09 \\
\hline UGC9128 & 2.24 & 7.75 & 49.8 & 23.91 & $\mathrm{~L}$ & $-3.379 \pm 0.307$ & $39.66 \pm 0.31$ & $39.88 \pm 0.31$ & $40.18 \pm 0.44$ & K08,D09 \\
\hline UGC9240 & 2.8 & 7.95 & 54.6 & 39.99 & $\mathrm{~L}$ & $-2.891 \pm 0.101$ & $40.19 \pm 0.09$ & $39.97 \pm 0.10$ & $40.47 \pm 0.12$ & K08,D09 \\
\hline Mrk475 & 9.66 & 7.97 & 11.69 & 10.43 & $\mathrm{~L}$ & $-1.566 \pm 0.101$ & $40.86 \pm 0.09$ & $40.06 \pm 0.11$ & $40.99 \pm 0.13$ & K08,D09 \\
\hline UGC9992 & 9.17 & 7.88 & 48.65 & 45.64 & $\mathrm{~L}$ & $-3.378 \pm 0.101$ & $39.59 \pm 0.09$ & $39.30 \pm 0.10$ & $39.83 \pm 0.12$ & K08,D09 \\
\hline ESO347-G17 & 10.04 & 7.9 & 41.4 & 40.39 & $\mathrm{~L}$ & $-2.779 \pm 0.101$ & $40.07 \pm 0.09$ & $39.73 \pm 0.10$ & $40.26 \pm 0.12$ & K08,D09 \\
\hline UGC12613 & 0.76 & 7.93 & 150.4 & 86.51 & $\mathrm{~L}$ & $-3.891 \pm 0.101$ & $39.51 \pm 0.09$ & $39.69 \pm 0.10$ & $40.01 \pm 0.12$ & K08,D09 \\
\hline
\end{tabular}


Table 1

(Continued)

\begin{tabular}{|c|c|c|c|c|c|c|c|c|c|c|}
\hline Name $^{\mathrm{a}}$ & $\begin{array}{c}D^{\mathrm{b}} \\
(\mathrm{Mpc})\end{array}$ & $12+\log (\mathrm{O} / \mathrm{H})^{\mathrm{c}}$ & $\begin{array}{c}R_{25}^{\mathrm{d}} \\
\left({ }^{\prime \prime}\right)\end{array}$ & $\begin{array}{c}R_{\mathrm{H} \alpha}{ }^{\mathrm{e}} \\
\left(^{\prime \prime}\right) \\
\end{array}$ & Ref. & $\begin{array}{c}\log \left(\Sigma_{\mathrm{SFR}}\right)^{\mathrm{f}} \\
\left(M_{\odot} \mathrm{yr}^{-1} \mathrm{kpc}^{-2}\right)\end{array}$ & $\begin{array}{c}\log \left(\Sigma_{70}\right)^{\mathrm{g}} \\
\left(\mathrm{erg} \mathrm{s}^{-1} \mathrm{kpc}^{-2}\right) \\
\end{array}$ & $\begin{array}{c}\log \left(\Sigma_{160}\right)^{\mathrm{g}} \\
\left(\mathrm{erg} \mathrm{s}^{-1} \mathrm{kpc}^{-2}\right)\end{array}$ & $\begin{array}{c}\log \left(\Sigma_{\mathrm{TIR}}\right)^{\mathrm{g}} \\
\left(\mathrm{erg} \mathrm{s}^{-1} \mathrm{kpc}^{-2}\right)\end{array}$ & Ref. \\
\hline UGCA442 & 4.27 & 7.72 & 106.5 & 101.4 & $\mathrm{~L}$ & $-3.500 \pm 0.101$ & $38.91 \pm 0.09$ & $38.89 \pm 0.10$ & $39.30 \pm 0.12$ & K08,D09 \\
\hline HolmII & 3.39 & 7.88 & 238.3 & 224. & $\mathrm{~L}, \mathrm{~S}$ & $-2.976 \pm 0.101$ & $39.71 \pm 0.09$ & $39.43 \pm 0.10$ & $39.95 \pm 0.11$ & K09,D07 \\
\hline M81DwA & 3.55 & 7.49 & 0.01 & 39.3 & $\mathrm{~L}, \mathrm{~S}$ & $-3.595 \pm 0.101$ & $39.83 \pm 0.09$ & $39.46 \pm 0.10$ & $40.10 \pm 0.11$ & K09,D07 \\
\hline DDO053 & 3.56 & 7.82 & 46.45 & 43.2 & $\mathrm{~L}, \mathrm{~S}$ & $-2.493 \pm 0.101$ & $40.18 \pm 0.09$ & $39.92 \pm 0.10$ & $40.44 \pm 0.11$ & K09,D07 \\
\hline HolmI & 3.84 & 8.00 & 108.9 & 83. & $\mathrm{~L}, \mathrm{~S}$ & $-3.302 \pm 0.101$ & $39.62 \pm 0.09$ & $39.60 \pm 0.10$ & $39.96 \pm 0.11$ & K09,D07 \\
\hline HolmIX & 3.28 & 7.8 & 75.35 & 29.4 & $\mathrm{~L}, \mathrm{~S}$ & $-2.712 \pm 0.101$ & $40.28 \pm 0.09$ & $40.22 \pm 0.10$ & $40.70 \pm 0.11$ & K09,D07 \\
\hline M81DwB & 7.08 & 8.02 & 26.15 & 20.1 & $\mathrm{~L}, \mathrm{~S}$ & $-2.418 \pm 0.101$ & $40.42 \pm 0.09$ & $40.47 \pm 0.10$ & $40.83 \pm 0.11$ & K09,D07 \\
\hline DDO154 & 4.3 & 7.78 & 90.6 & 63.5 & $\mathrm{~L}, \mathrm{~S}$ & $-3.346 \pm 0.101$ & $39.06 \pm 0.09$ & $39.43 \pm 0.10$ & $39.71 \pm 0.11$ & K09,D07 \\
\hline DDO165 & 4.57 & 7.80 & 104 & 25.3 & $\mathrm{~L}, \mathrm{~S}$ & $-2.659 \pm 0.101$ & $40.22 \pm 0.09$ & $40.20 \pm 0.10$ & $40.62 \pm 0.11$ & K09,D07 \\
\hline IC2574 & 2.8 & 7.93 & 395.5 & 305. & $\mathrm{~L}, \mathrm{~S}$ & $-3.237 \pm 0.101$ & $39.62 \pm 0.09$ & $39.58 \pm 0.10$ & $39.97 \pm 0.11$ & $\mathrm{~K} 08, \mathrm{D} 07$ \\
\hline NGC 5408 & 4.81 & 8.02 & 48.65 & 71.3 & S & $-1.991 \pm 0.101$ & $40.69 \pm 0.09$ & $40.19 \pm 0.10$ & $40.93 \pm 0.11$ & K09,D07 \\
\hline IZw18 & 12.6 & 7.19 & 8.85 & 8.39 & G03 & $-1.349 \pm 0.153$ & $40.51 \pm 0.15$ & $40.51 \pm 0.32$ & $40.98 \pm 0.32$ & G03,E08 \\
\hline Tol65 & 34 & 7.45 & 10.45 & 4.85 & G03 & $-0.957 \pm 0.153$ & $40.87 \pm 0.16$ & $40.99 \pm 0.32$ & $41.63 \pm 0.32$ & G03,E08 \\
\hline UGC4483 & 3.2 & 7.55 & 33.65 & 16.35 & G03 & $-1.939 \pm 0.154$ & $40.43 \pm 0.15$ & $39.95 \pm 0.19$ & $40.60 \pm 0.19$ & K08,E08 \\
\hline ESO146-G14 & 23.8 & 7.66 & 84.6 & 48.84 & A & $>-4.506 \pm 0.156$ & $39.45 \pm 0.15$ & $39.30 \pm 0.17$ & $39.72 \pm 0.17$ & $\ldots, \mathrm{E} 08$ \\
\hline Mrk178 & 4.7 & 7.82 & 36.9 & 36 & G03 & $-2.141 \pm 0.154$ & $39.97 \pm 0.15$ & $39.46 \pm 0.16$ & $40.07 \pm 0.16$ & K08,E08 \\
\hline Mrk153 & 41 & 7.83 & 24.4 & 14.09 & A & $>-2.491 \pm 0.153$ & $40.91 \pm 0.15$ & $40.34 \pm 0.16$ & $41.14 \pm 0.16$ & $\ldots, \mathrm{E} 08$ \\
\hline UM462 & 13.4 & 7.91 & 19.35 & 11.17 & $\mathrm{~A}$ & $>-1.691 \pm 0.153$ & $41.72 \pm 0.15$ & $41.15 \pm 0.15$ & $41.95 \pm 0.15$ & $\ldots$, E08 \\
\hline Haro11 & 87 & 7.92 & 14.7 & 4.3 & $\mathrm{H}$ & $0.604 \pm 0.153$ & $43.28 \pm 0.15$ & $42.54 \pm 0.15$ & $43.76 \pm 0.15$ & S06,E08 \\
\hline UGC4393 & 35 & 7.95 & 67.15 & 33.72 & J04 & $-2.195 \pm 0.153$ & $40.76 \pm 0.15$ & $40.98 \pm 0.15$ & $41.29 \pm 0.15$ & J04,E08 \\
\hline POX4 & 52 & 7.96 & 16.71 & 14.6 & G03 & $-1.238 \pm 0.153$ & $41.28 \pm 0.15$ & $40.64 \pm 0.15$ & $41.59 \pm 0.16$ & G03,E08 \\
\hline Tol2138-405 & 246 & 8.01 & 15.5 & 11.14 & A & $-1.956 \pm 0.153$ & $40.94 \pm 0.15$ & $40.55 \pm 0.16$ & $41.44 \pm 0.16$ & $\ldots, \mathrm{E} 08$ \\
\hline NGC 4861 & 15.2 & 8.01 & 119.5 & 118.5 & G03 & $-2.739 \pm 0.153$ & $40.01 \pm 0.15$ & $39.53 \pm 0.15$ & $40.30 \pm 0.15$ & $\mathrm{G} 03, \mathrm{E} 08$ \\
\hline Mrk206 & 25.4 & 8.04 & 18.1 & 10.45 & A & $>-1.371 \pm 0.153$ & $41.89 \pm 0.15$ & $41.42 \pm 0.15$ & $42.19 \pm 0.15$ & $\ldots$, E08 \\
\hline UM448 & 87 & 8.06 & 13.25 & 7.65 & $\mathrm{~A}$ & $>-0.632 \pm 0.153$ & $42.65 \pm 0.15$ & $42.16 \pm 0.15$ & $42.94 \pm 0.15$ & $\ldots, \mathrm{E} 08$ \\
\hline SHOC391 & 106 & 8.06 & 8.49 & 4.9 & A & $-0.228 \pm 0.153$ & $42.16 \pm 0.15$ & $41.43 \pm 0.16$ & $42.79 \pm 0.16$ & K04,E08 \\
\hline Mrk1450 & 20 & 7.99 & 13 & 5.1 & $\mathrm{C} 07$ & $-0.586 \pm 0.108$ & $41.92 \pm 0.15$ & $41.22 \pm 0.16$ & $42.19 \pm 0.16$ & $\mathrm{C} 07, \mathrm{E} 08$ \\
\hline SBS0335 - 052 & 57 & 7.25 & 15 & 4.1 & $\mathrm{C} 07$ & $-0.721 \pm 0.108$ & $41.33 \pm 0.15$ & $41.05 \pm 0.32$ & $42.26 \pm 0.32$ & C07,E08 \\
\hline VIIZw403 & 4.3 & 7.71 & 43.35 & 6.0 & $\mathrm{C} 07$ & $-1.107 \pm 0.108$ & $41.95 \pm 0.15$ & $41.33 \pm 0.40$ & $42.12 \pm 0.40$ & $\mathrm{C} 07, \mathrm{E} 08$ \\
\hline HSO822+ 3542 & 11 & 7.4 & 8 & 4.1 & $\mathrm{C} 07$ & $-1.192 \pm 0.108$ & $41.22 \pm 0.15$ & $41.00 \pm 0.32$ & $41.53 \pm 0.32$ & $\mathrm{C} 07, \mathrm{E} 08$ \\
\hline UGCA292 & 3.1 & 7.27 & 30 & 5.0 & $\mathrm{C} 07$ & $-1.869 \pm 0.108$ & $40.89 \pm 0.31$ & $40.65 \pm 0.32$ & $41.11 \pm 0.39$ & $\mathrm{C} 07, \mathrm{E} 08$ \\
\hline UM461 & 13.4 & 7.8 & 9.08 & 5.0 & $\mathrm{C} 07$ & $-0.892 \pm 0.108$ & $41.57 \pm 0.15$ & $40.69 \pm 0.21$ & $41.89 \pm 0.21$ & $\mathrm{C} 07, \mathrm{E} 08$ \\
\hline
\end{tabular}

Notes.

a Galaxy name, as listed in Dale et al. (2007), Dale et al. (2009), or Engelbracht et al. (2008).

b Distance in Mpc, rescaled, where necessary, to $H_{0}=70 \mathrm{~km} \mathrm{~s}^{-1} \mathrm{Mpc}^{-1}$, as reported in Dale et al. (2007), Kennicutt et al. (2008), and Engelbracht et al. (2008).

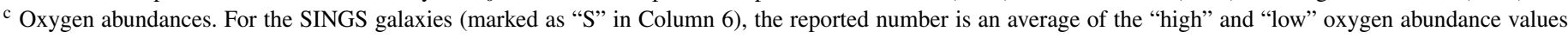

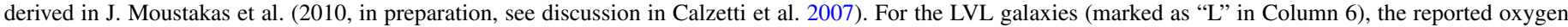

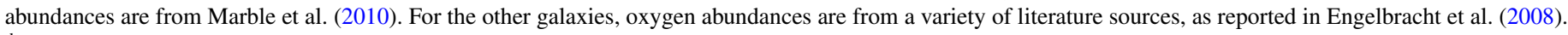

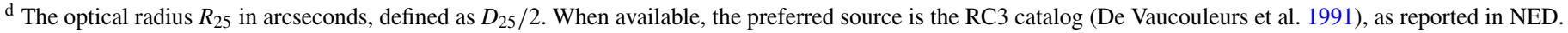

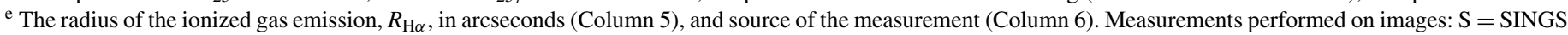

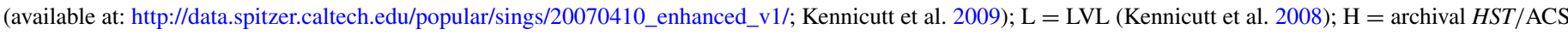

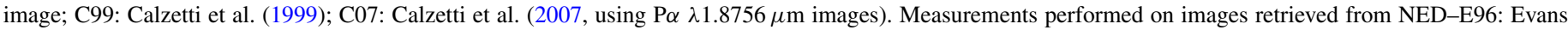

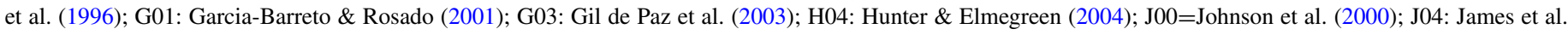

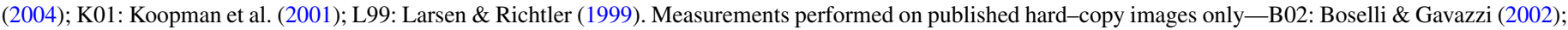

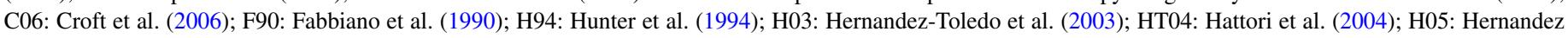
et al. (2005); M99: Mendez et al. (1999); R07: Robitaille et al. (2007). When no image is available, values adopted from Figure 1 are indicated with "A."

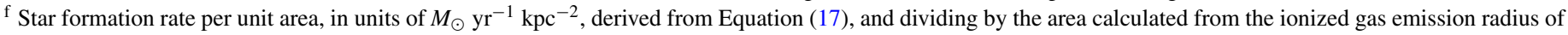
Column 5. The $\Sigma_{\mathrm{SFR}}$ of galaxies for which the H $\alpha$ flux information is missing (see last column of table) is marked as a lower limit.

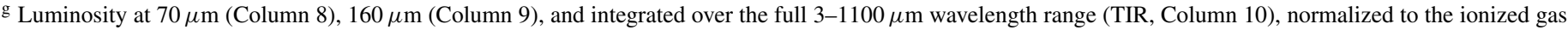
emission area, in units of $\operatorname{erg~s}^{-1} \mathrm{kpc}^{-2}$.

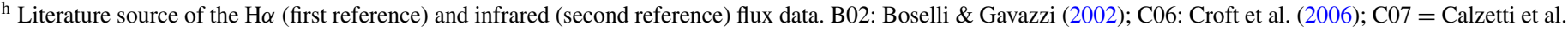

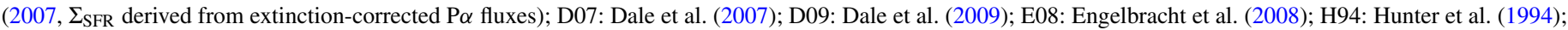

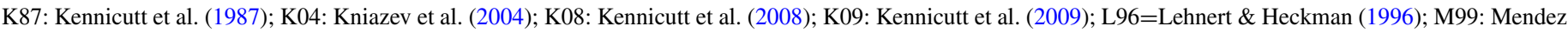
et al. (1999); M06: Moustakas \& Kennicutt (2006); P06: Perez-Gonzalez et al. (2006); S06: Schmitt et al. (2006); W99: Wang et al. (1999).

tal infrared luminosity, from $\sim 3 \times 10^{39}-3 \times 10^{45} \mathrm{erg} \mathrm{s}^{-1}$ (the derivation of fluxes in "MIPS-equivalent" bands for the LIRGs is discussed in the next section). We also explore one order of magnitude range in the $70-160 \mu \mathrm{m}$ luminosity ratio, from about $1 / 3$ to roughly 6 times in $L(70) / L(160)$. The LIRGs occupy a relatively small range in luminosity ratio, with values in the range $L(70) / L(160) \sim$
$2-5$, as expected for these warm (relatively to many galaxies) systems.

\section{LUMINOSITIES, SIZES, AND UNCERTAINTIES}

We derive total infrared luminosities, $L$ (TIR), in the range $3-1100 \mu \mathrm{m}$ for the galaxies in the LVL, SINGS, and MIPSGTO samples using Equation (4) in Dale \& Helou (2002). Most 
Table 2

Properties of Luminous Infrared Galaxies

\begin{tabular}{|c|c|c|c|c|c|c|c|c|c|}
\hline Name $^{\mathrm{a}}$ & $\begin{array}{c}D^{\mathrm{b}} \\
(\mathrm{Mpc})\end{array}$ & $\begin{array}{l}R_{25}{ }^{\mathrm{c}} \\
\left(^{\prime \prime}\right) \\
\end{array}$ & $\begin{array}{c}R_{\mathrm{H} \alpha}{ }^{\mathrm{d}} \\
\left(^{\prime \prime}\right) \\
\end{array}$ & $\begin{array}{c}\log \left(\Sigma_{\mathrm{SFR}}\right)^{\mathrm{e}} \\
\left(M_{\odot} \mathrm{yr}^{-1} \mathrm{kpc}^{-2}\right)\end{array}$ & $\begin{array}{c}\log \left(\Sigma_{24}\right)^{\mathrm{f}} \\
\left(\mathrm{erg} \mathrm{s}^{-1} \mathrm{kpc}^{-2}\right)\end{array}$ & $\begin{array}{c}\log \left(\Sigma_{70}\right)^{\mathrm{f}} \\
\left(\operatorname{erg~s}^{-1} \mathrm{kpc}^{-2}\right)\end{array}$ & $\begin{array}{c}\log \left(\Sigma_{160}\right)^{\mathrm{f}} \\
\left(\mathrm{erg} \mathrm{s}^{-1} \mathrm{kpc}^{-2}\right)\end{array}$ & $\begin{array}{c}\log \left(\Sigma_{\mathrm{TIR}}\right)^{\mathrm{f}} \\
\left(\mathrm{erg} \mathrm{s}^{-1} \mathrm{kpc}^{-2}\right)\end{array}$ & $\begin{array}{c}\log \left(\Sigma_{3.6}\right)^{\mathrm{g}} \\
\left(\mathrm{erg} \mathrm{s}^{-1} \mathrm{kpc}^{-2}\right)\end{array}$ \\
\hline NGC 0023 & 63.8 & 62.7 & 10. & $0.602 \pm 0.154$ & $43.36 \pm 0.15$ & $43.93 \pm 0.16$ & $43.59 \pm 0.16$ & $44.19 \pm 0.18$ & $43.03 \pm 0.15$ \\
\hline MCG+12-02-001 & 68.9 & 6.6 & 4.9 & $1.179 \pm 0.154$ & $43.92 \pm 0.15$ & $44.39 \pm 0.16$ & $43.92 \pm 0.16$ & $44.63 \pm 0.18$ & $\ldots \pm \ldots$ \\
\hline NGC 0633 & 72.7 & 38.6 & 4.9 & $0.807 \pm 0.154$ & $43.56 \pm 0.15$ & $43.97 \pm 0.16$ & $43.52 \pm 0.16$ & $44.24 \pm 0.18$ & $42.68 \pm 0.15$ \\
\hline UGC1845 & 66.4 & 36.1 & 6.2 & $0.428 \pm 0.154$ & $43.19 \pm 0.15$ & $43.88 \pm 0.16$ & $43.49 \pm 0.16$ & $44.09 \pm 0.18$ & $42.60 \pm 0.15$ \\
\hline NGC 1614 & 62.7 & 39.5 & 13.2 & $0.606 \pm 0.146$ & $43.33 \pm 0.15$ & $43.49 \pm 0.15$ & $42.97 \pm 0.15$ & $43.85 \pm 0.15$ & $42.09 \pm 0.15$ \\
\hline UGC3351 & 65.2 & 47.6 & 12.7 & $-0.287 \pm 0.154$ & $42.48 \pm 0.15$ & $43.44 \pm 0.15$ & $43.06 \pm 0.16$ & $43.62 \pm 0.17$ & $42.16 \pm 0.15$ \\
\hline NGC 2369 & 47.1 & 106.5 & 17.5 & $-0.169 \pm 0.148$ & $42.60 \pm 0.15$ & $43.30 \pm 0.15$ & $42.87 \pm 0.15$ & $43.50 \pm 0.15$ & $42.24 \pm 0.15$ \\
\hline NGC 2388 & 61.9 & 30.0 & 13.3 & $0.045 \pm 0.154$ & $42.80 \pm 0.15$ & $43.42 \pm 0.16$ & $42.94 \pm 0.16$ & $43.62 \pm 0.18$ & $42.01 \pm 0.15$ \\
\hline MCG+02-20-003 & 72.4 & 19.0 & 2.8 & $0.982 \pm 0.154$ & $43.75 \pm 0.15$ & $44.51 \pm 0.16$ & $44.11 \pm 0.16$ & $44.70 \pm 0.18$ & $42.84 \pm 0.15$ \\
\hline NGC 3110 & 78.7 & 46.5 & 20. & $0.012 \pm 0.154$ & $42.77 \pm 0.15$ & $43.50 \pm 0.15$ & $43.15 \pm 0.16$ & $43.72 \pm 0.17$ & $42.24 \pm 0.15$ \\
\hline NGC 3256 & 35.5 & 114.1 & 23.3 & $0.434 \pm 0.146$ & $43.17 \pm 0.15$ & $43.55 \pm 0.15$ & $43.02 \pm 0.15$ & $43.81 \pm 0.15$ & $42.17 \pm 0.15$ \\
\hline NGC 3690/IC694 & 51.1 & 85.2 & 16.2 & $1.008 \pm 0.154$ & $43.72 \pm 0.15$ & $44.02 \pm 0.16$ & $43.31 \pm 0.16$ & $44.28 \pm 0.18$ & $42.56 \pm 0.15$ \\
\hline ESO320-G030 & 40.4 & 67.2 & 8.8 & $0.448 \pm 0.147$ & $43.22 \pm 0.15$ & $44.04 \pm 0.15$ & $43.53 \pm 0.15$ & $44.19 \pm 0.15$ & $42.51 \pm 0.15$ \\
\hline MCG-02-33-098 & 77.7 & 49.8 & 3.5 & $1.367 \pm 0.154$ & $44.12 \pm 0.15$ & $44.45 \pm 0.16$ & $43.93 \pm 0.16$ & $44.73 \pm 0.18$ & $43.33 \pm 0.17$ \\
\hline IC860 & 63.3 & 16.5 & 3.3 & $1.088 \pm 0.154$ & $43.85 \pm 0.15$ & $44.65 \pm 0.15$ & $43.94 \pm 0.16$ & $44.76 \pm 0.17$ & $42.83 \pm 0.15$ \\
\hline NGC 5135 & 55.9 & 77.1 & 7.4 & $0.638 \pm 0.154$ & $43.39 \pm 0.15$ & $43.97 \pm 0.16$ & $43.55 \pm 0.16$ & $44.20 \pm 0.18$ & $42.82 \pm 0.15$ \\
\hline NGC 5653 & 65.1 & 52.2 & 12.7 & $-0.076 \pm 0.154$ & $42.68 \pm 0.15$ & $43.31 \pm 0.15$ & $42.95 \pm 0.16$ & $43.55 \pm 0.17$ & $42.23 \pm 0.15$ \\
\hline NGC 5734 & 63.5 & 45.4 & 13.0 & $-0.379 \pm 0.154$ & $42.39 \pm 0.15$ & $43.20 \pm 0.15$ & $42.96 \pm 0.16$ & $43.45 \pm 0.17$ & $42.20 \pm 0.15$ \\
\hline IC4518 & 74.9 & 8.4 & 6.2 & $0.606 \pm 0.154$ & $43.36 \pm 0.15$ & $43.79 \pm 0.16$ & $43.35 \pm 0.16$ & $44.06 \pm 0.18$ & $\ldots \pm \ldots$ \\
\hline Zw049.057 & 63.3 & 26.8 & 13.0 & $-0.269 \pm 0.154$ & $42.50 \pm 0.15$ & $43.58 \pm 0.15$ & $43.00 \pm 0.16$ & $43.67 \pm 0.17$ & $41.52 \pm 0.15$ \\
\hline NGC 5936 & 65.1 & 43.4 & 8.4 & $0.315 \pm 0.154$ & $43.07 \pm 0.15$ & $43.58 \pm 0.15$ & $43.20 \pm 0.16$ & $43.84 \pm 0.17$ & $42.47 \pm 0.15$ \\
\hline IRAS17138-1017 & 81.2 & 22.5 & 5.1 & $0.929 \pm 0.154$ & $43.67 \pm 0.15$ & $44.19 \pm 0.16$ & $43.71 \pm 0.16$ & $44.42 \pm 0.18$ & $\ldots \pm \ldots$ \\
\hline IC4687/6 & 79.4 & 38.7 & 5.8 & $1.046 \pm 0.154$ & $43.78 \pm 0.15$ & $44.21 \pm 0.16$ & $43.76 \pm 0.16$ & $44.47 \pm 0.18$ & $42.65 \pm 0.15$ \\
\hline IC4734 & 73.5 & 38.7 & 5.6 & $0.626 \pm 0.154$ & $43.38 \pm 0.15$ & $44.12 \pm 0.15$ & $43.75 \pm 0.16$ & $44.33 \pm 0.17$ & $42.69 \pm 0.15$ \\
\hline NGC 6701 & 60.6 & 46.5 & 6.8 & $0.443 \pm 0.154$ & $43.21 \pm 0.15$ & $43.82 \pm 0.16$ & $43.43 \pm 0.16$ & $44.05 \pm 0.18$ & $42.70 \pm 0.15$ \\
\hline NGC 7130 & 70.7 & 45.4 & 11.7 & $0.210 \pm 0.154$ & $42.96 \pm 0.15$ & $43.54 \pm 0.16$ & $43.12 \pm 0.16$ & $43.77 \pm 0.18$ & $42.27 \pm 0.15$ \\
\hline IC5179 & 50.0 & 70.4 & 16.5 & $-0.062 \pm 0.154$ & $42.70 \pm 0.15$ & $43.34 \pm 0.16$ & $42.93 \pm 0.16$ & $43.56 \pm 0.18$ & $42.21 \pm 0.15$ \\
\hline NGC 7591 & 70.2 & 58.5 & 5.9 & $0.559 \pm 0.154$ & $43.32 \pm 0.15$ & $43.84 \pm 0.16$ & $43.43 \pm 0.16$ & $44.08 \pm 0.18$ & $42.73 \pm 0.15$ \\
\hline NGC 7771 & 61.2 & 75.4 & 30. & $0.080 \pm 0.154$ & $42.83 \pm 0.15$ & $43.52 \pm 0.15$ & $43.19 \pm 0.16$ & $43.76 \pm 0.17$ & $42.51 \pm 0.15$ \\
\hline
\end{tabular}

Notes.

${ }^{a}$ Galaxy name, as listed in Alonso-Herrero et al. (2006).

${ }^{\mathrm{b}}$ Distance in Mpc from Sanders et al. (2003) and Surace et al. (2004), rescaled to $H_{o}=70 \mathrm{~km} \mathrm{~s}^{-1} \mathrm{Mpc}^{-1}$.

c The optical radius $R_{25}$ in arcseconds. When available, the preferred source is the RC3 catalog (De Vaucouleurs et al. 1991), as reported in NED

d The radius of the ionized gas emission, $R_{\mathrm{H} \alpha}$, in arcseconds. When available, the $\mathrm{H} \alpha$ sizes are from the images in Hattori et al. (2004); otherwise they are from Alonso-Herrero et al. (2006), using HST NICMOS P $\alpha$ 1.8756 $\mu \mathrm{m}$ images. For NGC 1614, NGC 3256, NGC 3690, NGC 5653, and Zw049.057, the data are from Alonso-Herrero et al. (2000, 2001, 2002).

${ }^{\mathrm{e}} \mathrm{Star}$ formation rate per unit area, in units of $M_{\odot} \mathrm{yr}^{-1} \mathrm{kpc}^{-2}$, derived from Equation (17), and dividing by the area calculated from the ionized gas emission radius of Column 4.

${ }^{\mathrm{f}}$ Luminosity per unit area at $24 \mu \mathrm{m}, 70 \mu \mathrm{m}, 160 \mu \mathrm{m}$, and integrated over the full $8-1000 \mu \mathrm{m}$ wavelength range (TIR), in units of erg s ${ }^{-1} \mathrm{kpc}^{-2}$, using the ionized gas emission area. The luminosities in the MIPS bands are extrapolated from the IRAS measurements as described in Section 3 . Exceptions are NGC 1614 and NGC 3256, with MIPS data reported in Engelbracht et al. (2008), and NGC 2369, ESO320-G030, and Zw049.057, with IR SED published in Rieke et al. (2009). $\mathrm{g}$ Luminosity per unit area at $3.6 \mu \mathrm{m}$, in units of $\mathrm{erg} \mathrm{s}^{-1} \mathrm{kpc}^{-2}$, extrapolated from the $K_{S}$-band luminosity, as described in Section 3 . $\mathrm{K}_{S}-$ band luminosities are from Two Micron All Sky Survey (2MASS), as reported in NED, and corrected for the effect of both Galactic foreground (Schlegel et al. 1998) and internal extinction.

of the galaxies are also bright enough to have IRAS detections, at least at 25,60 , and $100 \mu \mathrm{m}$, but we concentrate on the MIPS data alone. All infrared fluxes are used in this work as directly measured (different from the approach of, e.g., Engelbracht et al. 2008, who use color-corrected fluxes).

For most of the LIRGs, only IRAS infrared data are available, typically with detections at 25,60, and $100 \mu \mathrm{m}$. Exceptions are NGC 1614 and NGC 3256, which have published MIPS measurements (Engelbracht et al. 2008), and NGC 2369, ESO320G030, and Zw049.057, for which Rieke et al. (2009) published infrared spectral energy distributions (SEDs). Since our reference luminosities are those in the MIPS bands, we need to interpolate the IRAS band fluxes of all the other LIRGs to derive MIPS-equivalent ones. The difference between the luminosity at MIPS $24 \mu \mathrm{m}$ and at IRAS $25 \mu \mathrm{m}$ is small (see also Kennicutt et al. 2009), and we adopt the best fit:

$$
\log [L(24)]=\log [L(25)]-0.028,
$$

where $L(\lambda)=4 \pi d^{2}(c / \lambda) f_{v}(\lambda)$, in units of erg s$s^{-1}$, is the monochromatic luminosity at wavelength $\lambda, f_{v}(\lambda)$ is the flux density per unit frequency, and $d$ is the galaxy's distance. The difference between the $60 \mu \mathrm{m}$ IRAS band and the $70 \mu \mathrm{m}$ MIPS band is larger than between the $25 \mu \mathrm{m}$ and $24 \mu \mathrm{m}$ bands, and we use a polynomial interpolation between the 60 and $100 \mu \mathrm{m}$ IRAS bands to derive a $70 \mu \mathrm{m}$ equivalent flux density:

$$
f_{v}(70)=f_{v}(60)\left(a_{0}+a_{1} x+a_{2} x^{2}+a_{3} x^{3}+a_{4} x^{4}+a_{5} x^{5}\right),
$$

with $f_{v}(\lambda)$ the flux density in Jy, $x=f_{v}(60) / f_{v}(100)$, and the vector $a_{n}=[1.5078,0.9356,-3.8900,4.0576,-1.7617$, 0.2712]. Tests using starburst galaxies from the MIPS-GTO sample with IRAS $60 / 100$ colors similar to the LIRGs $\left(0.4 \leqslant f_{v}(60) / f_{\nu}(100) \leqslant 1.3\right)$ show that the above interpola- 


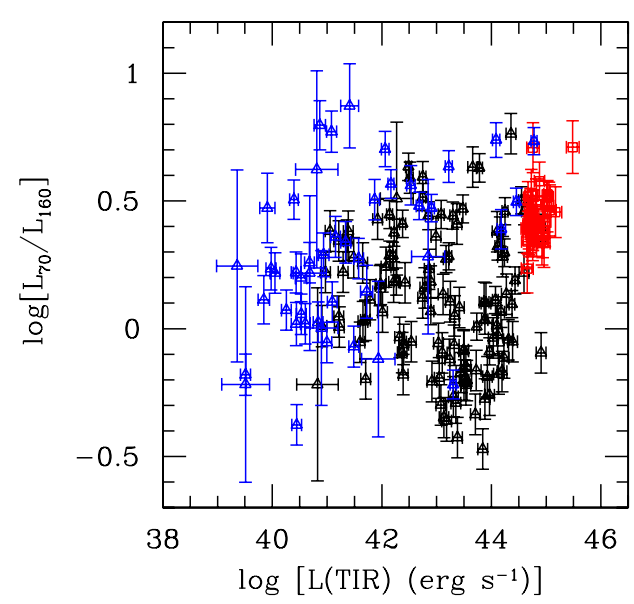

Figure 1. Logarithm of the ratio of 70-160 $\mu \mathrm{m}$ luminosity for the 189 galaxies in our sample, as a function of the total infrared luminosity TIR. The sample covers almost 6 orders of magnitude in infrared luminosity. Symbol colors identify the star-forming and starburst high-metallicity (black symbols) and low-metallicity (blue symbols) galaxies, and the LIRGs (red).

(A color version of this figure is available in the online journal.)

tion reproduces the observed $L(70)$ with a typical uncertainty of less than $10 \%$ (i.e., less than 0.04 dex in logarithmic scale). We also compare our interpolated values with MIPS-equivalent fluxes derived from the template SEDs of Rieke et al. (2009), by matching LIRGs to the templates according to TIR luminosity and SED shape (using the IRAS fluxes). We typically find that the values derived from the templates differ from those derived from Equations (1) and (2) by $\sim 1 \%-5 \%$ at $24 \mu \mathrm{m}$ and less than $12 \%$ at $70 \mu \mathrm{m}$.

We use the SED templates also to extrapolate fluxes at $160 \mu \mathrm{m}$ for the LIRGs with only IRAS measurements. Nine of the 29 LIRGs have long-wavelength measurements at $850 \mu \mathrm{m}$ from SCUBA on the JCMT (Dunne et al. 2000) and one, NGC 3690/ IC694, at $1.2 \mathrm{~mm}$ from the IRAM-30 $\mathrm{m}$ telescope (Braine \& Dumke 1998). Interpolations between these long wavelengths and the IRAS $100 \mu \mathrm{m}$ band to recover the MIPS $160 \mu \mathrm{m}$ emission are complicated by the presence of a cool, $T \approx 20 \mathrm{~K}$, dust component in the IR emission of LIRGs (e.g., Dunne \& Eales 2001). The MIPS $160 \mu \mathrm{m}$ band is close to the emission peak of this cool dust component, thus any interpolation will be subject to the uncertainty of the relative, and unknown, ratio of the cool and warm dust emission components. A simple linear interpolation between $100 \mu \mathrm{m}$ and $450 \mu \mathrm{m}$ (using the roughly constant $450-850$ ratio of 7.9 found by Dunne \& Eales 2001) provides estimated $160 \mu \mathrm{m}$ fluxes that are between $30 \%$ and $75 \%$ lower than those estimated from the template SEDs, in agreement with the presence of the $20 \mathrm{~K}$ emitting dust component. To avoid a significant underestimate in the $160 \mu \mathrm{m}$ flux, we adopt the results from the extrapolation of the SED templates as our "bona fide" $160 \mu \mathrm{m}$ fluxes for the LIRGs. The resulting $70-160 \mu \mathrm{m}$ luminosity ratio for the LIRGs has median value $L(70) / L(160) \sim 2.7$, to be compared with the median ratio $L(70) / L(160) \sim 3.5$ of the five galaxies for which direct MIPS measurements are available. The difference is only $\sim 30 \%$ (0.11 dex), implying that our procedure overestimates $L(160)$ by that amount at most. This is well within the typical uncertainty for our measurements.

For the wavelength-integrated infrared luminosity $L(\mathrm{TIR})$ of the LIRGs, we compare the results of Equation (4) of Dale \& Helou (2002), using the MIPS-equivalent fluxes obtained with the SED templates and Equations (1) and (2) with the results of their Equation (5), using the IRAS bands. The two equations are known to provide different $L(\mathrm{TIR})$ values for the more quiescently star-forming galaxies and for galaxies with cooler dust (Dale \& Helou 2002), because the MIPS $160 \mu \mathrm{m}$ band traces cool dust more accurately than the IRAS $100 \mu \mathrm{m}$ band (the two being the longest wavelength bands from Spitzer and IRAS, respectively). The offset is dependent on the 60/ 100 color (see, e.g., Kennicutt et al. 2009), and L(TIR) values obtained with the two methods for the starburst galaxies from the sample of Engelbracht et al. (2008) provide an empirical correction:

$$
\begin{aligned}
\log \left[L(\mathrm{TIR})_{\mathrm{MIPS}}\right]= & L\left[L(\mathrm{TIR})_{\mathrm{IRAS}}\right] \\
& -0.35 \log \left[f_{v}(60) / f_{v}(100)\right]-0.15,
\end{aligned}
$$

which gives consistent $L$ (TIR) values with a scatter typically less than 25\%. A list of "MIPS-equivalent" quantities for the LIRGs is given in Table 2.

We finally apply Equations (1)-(3) and the SED templates of Rieke et al. (2009) to recover, from the IRAS measurements, the 24, 70, and $160 \mu \mathrm{m}$ fluxes, and the TIR luminosity of NGC 3034, ${ }^{21}$ a starburst galaxy included in both the SINGS and LVL samples, but which is saturated in all three MIPS bands.

Galaxy-integrated emission line data include $\mathrm{H} \alpha+[\mathrm{N}$ II $]$ for the LVL (Kennicutt et al. 2008) and SINGS (Kennicutt et al. 2009) samples; those papers also report the $[\mathrm{N} \mathrm{II}] / \mathrm{H} \alpha$ ratios appropriate for each galaxy. Typical uncertainties on the final integrated $\mathrm{H} \alpha$ fluxes are about $10 \%-15 \%$. Similar data are also available for 39 of the MIPS-GTO galaxies, mostly from the literature (Engelbracht et al. 2008), although measurements for 11 galaxies are from the LVL data (Kennicutt et al. 2008), and, for 10 galaxies, $\mathrm{P} \alpha$ fluxes are also available (Calzetti et al. 2007). Larger uncertainties, of order $20 \%$, are expected for the $\mathrm{H} \alpha$ fluxes of the starbursts, because of the non-uniform nature of the data. Finally, the only ionized gas data readily available for the LIRGs are the P $\alpha$ data presented in Alonso-Herrero et al. (2006), together with estimates of the dust extinction values.

Uncertainties for the line-emitting region sizes range from about $10 \%$ for the galaxies for which we perform a direct measure on the images (mostly the LVL and SINGS samples), to $\sim 15 \%-20 \%$ for the 34 MIPS-GTO galaxies with available FITS images, to about $20 \%-30 \%$ for the starburst galaxies for which images were available only in hard-copy published form. For the LIRGs, the region size information is from Hattori et al. (2004) and Alonso-Herrero et al. (2006), with an estimated uncertainty of about $20 \%$. For 11 of the MIPS-GTO starbursts, line-emission images are not available, either from archives (e.g., NED) or from the literature. We observe, however, that the size of the star-forming region decreases relative to the size of the stellar emission, defined by $R_{25}$, as the infrared luminosity surface density $($ IRSD $=L($ TIR) $/$ area) increases (Figure 2). This had already been reported by Wang \& Helou (1992) and Lehnert \& Heckman (1996), as an effect of increasing compactness of the region for increasing SFSD. Indeed, the infrared luminosity traces the SFR fairly accurately at high luminosities (Hunter et al. 1986; Devereux \& Young 1990; Lehnert \& Heckman 1996), and only at low luminosities does this correlation break, i.e., in a regime where the dust column density is low and most of the star formation light is unprocessed by dust; a similar trend

\footnotetext{
21 We recover the following flux densities for NGC 3034: $f_{v}(24)=287.4 \mathrm{Jy}$, $f_{v}(70)=1622 \mathrm{Jy}, f_{v}(160)=639.6 \mathrm{Jy}$; the IRAS 25, 60, and $100 \mu \mathrm{m}$ data used to derive the "MIPS-equivalent" fluxes are from Sanders et al. (2003).
} 


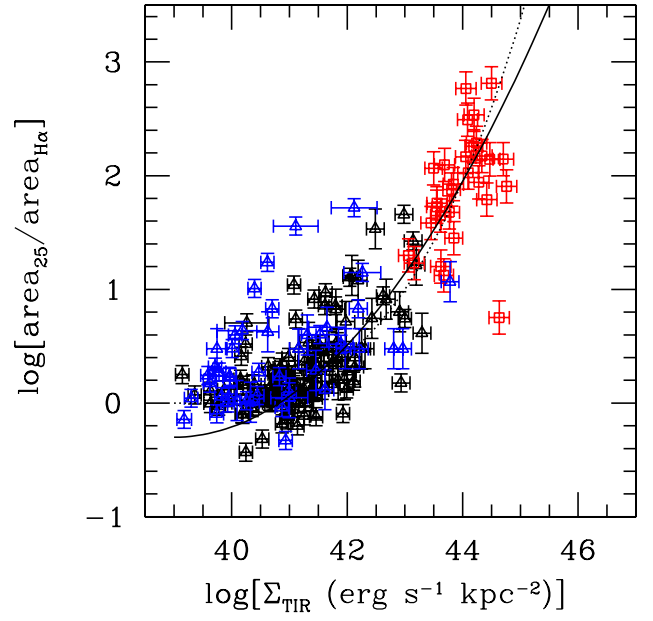

Figure 2. Ratio of the area defined by the $R_{25}$ radius to the ionized gas $(\mathrm{H} \alpha)$ emitting area as a function of the infrared luminosity surface density (IRSD: $\Sigma_{\mathrm{TIR}}=L(\mathrm{TIR}) /$ area) for our sample galaxies, where the area used to normalize the infrared luminosity is area $\mathrm{H} \alpha$. Symbol colors are as in Figure 1. Two indicative mean trends are shown for the high-metallicity data points; the trends are not fits to the data.

(A color version of this figure is available in the online journal.)

to that of Figure 2 is, in fact, observed when the IRSD is replaced by the SFSD in the correlation with area ${ }_{25} / \operatorname{area}_{\mathrm{H} \alpha}$. We use the median value measured for starbursts $\left(\operatorname{area}_{25} / \operatorname{area}_{\mathrm{H} \alpha} \sim 3\right)$ to ascribe a star-forming size to the 11 MIPS-GTO starbursts for which only $R_{25}$ is available (Table 1 ).

In addition to normalizing luminosities by the emission area, we also normalize by total stellar mass in the galaxy as a way to remove distance dependencies. The $3.6 \mu \mathrm{m}$ emission from the galaxies is used here as a proxy for the stellar mass, since, at these long wavelengths, the light is dominated by the photospheric emission from low-mass stars; hence the measurement is insensitive to the details of the galaxy's star formation history, and dust attenuation is small (see, e.g., Nishiyama et al. 2009, for a discussion on the dust extinction curve at these wavelengths). Furthermore, the contribution from dust emission is also negligible, even if the $3.3 \mu \mathrm{m} \mathrm{PAH}$ emission band is included in the Spitzer-IRAC $3.6 \mu \mathrm{m}$ band (Pahre et al. 2004; Calzetti et al. 2005; Dale et al. 2007; Engelbracht et al. 2008; Leroy et al. 2008). The list of $3.6 \mu \mathrm{m}$ fluxes for each galaxy is given in the same publications where the MIPS data are listed. For the LIRGs, we use the $K_{S}$-band measurements (retrieved from NED), which, after extinction correction using the starburst curve of Calzetti et al. (2000), we extrapolate to $3.6 \mu \mathrm{m}$-equivalent fluxes using the starburst models from Starburst99 (2007 Update; Leitherer et al. 1999), with ages in the range $20-100 \mathrm{Myr}$; we note that at these long wavelengths the spectral slope is basically insensitive to age, up to about $10 \mathrm{Gyr}$. The inferred " $3.6 \mu \mathrm{m}$ equivalent" luminosities for the LIRGs correspond to extinction-corrected values, which are the appropriate values to use in our analysis. In the presence of significant extinction at $3.6 \mu \mathrm{m}$, the observed luminosities are expected to be lower than those listed in Table 2 .

\section{REFERENCE STAR FORMATION RATES}

When testing the suitability of a luminosity (monochromatic and non-monochromatic) as an SFR indicator, the first task is to decide what the "reference" SFR tracer is going to be for the selected sample. In this work, we give preference to "reference" SFR tracers which incorporate infrared luminosities, as the goal is to test the $70 \mu \mathrm{m}$ luminosity as a SFR indicator, and remaining close to this wavelength allows us to avoid biases due to large differences in the dust optical depth probed.

In addition to the historical SFR tracers based on the bolometric infrared emission (Hunter et al. 1986; Lonsdale Persson \& Helou 1987; Rowan-Robinson \& Crawford 1989; Devereux \& Young 1990; Sauvage \& Thuan 1992; Lehnert \& Heckman 1996; Kennicutt 1998), a number of calibrations have appeared in the literature in recent years based on the Spitzer MIPS $24 \mu \mathrm{m}$ monochromatic band emission, or, equivalently, on the IRAS $25 \mu \mathrm{m}$ emission (e.g., Calzetti et al. 2005, 2007; Wu et al. 2005; Alonso-Herrero et al. 2006; Perez-Gonzalez et al. 2006; Zhu et al. 2008; Rieke et al. 2009; Kennicutt et al. 2009). Composite indicators based on a combination of the $\mathrm{H} \alpha$ optical recombination line emission or UV stellar continuum and one infrared band emission, at $8 \mu \mathrm{m}$ or $24 \mu \mathrm{m}$, have also been calibrated (Calzetti et al. 2007; Kennicutt et al. 2007, 2009; Zhu et al. 2008; Leroy et al. 2008), since the infrared bands capture the SFR from regions obscured by dust, while the optical recombination line (or UV stellar continuum) captures the SFR from regions that are relatively unobscured by dust. The composite indicators are indeed the most appropriate to use in the case of low-metallicity galaxies, where a large fraction of the star formation emerges virtually unaffected by dust reprocessing.

We intentionally avoid SFR indicators based on the total infrared emission, $L$ (TIR), because the $70 \mu \mathrm{m}$ wavelength region is close to the peak emission for many galaxies, and $L(70)$ is thus a significant contributor to $L(\mathrm{TIR})$; this causes a degeneracy between $L(70)$ and $L(T I R)$. Furthermore, the dust contributing to the long-wavelength infrared emission can be heated by evolved stellar populations, as well as by the currently star-forming ones; the "extra" contribution to the infrared emission will produce an overestimate of the true SFR (Lonsdale Persson \& Helou 1987; Sauvage \& Thuan 1992; Buat \& Xu 1996). Although the magnitude of this contribution is uncertain and its impact on the infrared emission not unambiguously established (see, e.g., Kewley et al. 2002), its potential dependence on the relative ratio of evolved/star-forming stellar populations in the galaxy is sufficient reason for not using $L$ (TIR) as a reference SFR in the present work.

\subsection{The Infrared Spectral Energy Distribution of Star-forming Galaxies}

Most findings indicate that the correlation between $L(24)$ and SFR is nonlinear, in the sense that the infrared emission at $24 \mu \mathrm{m}$ is overluminous in more actively star-forming systems relative to a linear scaling between $L(24)$ and SFR. Two nonexclusive interpretations are possible for this result. The first interpretation attributes the overluminosity at the bright end of the $24 \mu \mathrm{m}$ emission to the higher mean dust temperatures of more active systems (Calzetti et al. 2007); in this case, the infrared SED becomes "bluer" and $L(24) / L(T I R)$ increases at higher SFRs. The second interpretation suggests that, as the dust optical depth increases with increasing SFR, standard dust extinction correction methods based, e.g., on the Balmer decrement or other hydrogen line ratios, are insufficient to recover the intrinsic values of optical or near-infrared indicators ( $\mathrm{H} \alpha, \mathrm{P} \alpha$, etc.), thus artificially leading to the interpretation that $L(24)$ is overluminous relative to those indicators (Rieke et al. 2009).

In order to investigate the nature of the $L(24)$ overluminosity, we plot the ratio $L(24) / L(T I R)$ as a function of the ILSD (Figure 3 , left). A general increase of the $24 \mu \mathrm{m}$ luminosity as a 

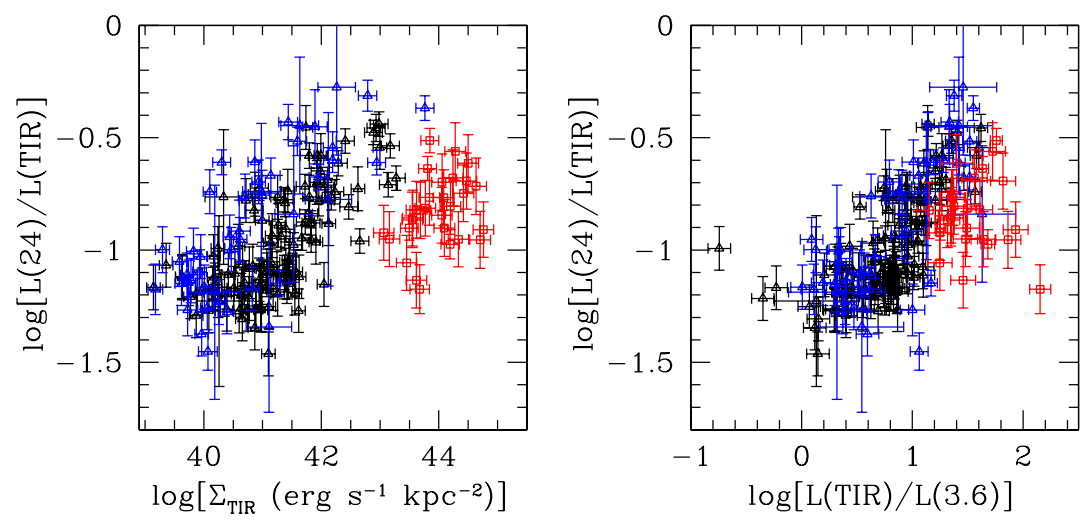

Figure 3. $L(24) / L($ TIR) ratio as a function of the IRSD (left) and of the infrared luminosity per unit stellar luminosity at $3.6 \mu \mathrm{m}$ (a proxy for mass in stars, right) for the galaxies in our sample. The color scheme for the data points is as in Figure 1.

(A color version of this figure is available in the online journal.)

function of IRSD is observed for normal and starburst galaxies, both at low and high metallicities; however, a significant break in the trend is observed for the LIRGs in our sample. These galaxies show significantly "cooler" infrared SEDs (lower $L(24) / L$ (TIR), by roughly $0.4-0.5 \mathrm{dex}$ ) than one would infer from their LSD if they followed the same trend as normal starforming and starburst galaxies. Low-metallicity galaxies mark a trend similar to that of the high-metallicity ones, but at lower average IRSD, as expected if the low-metallicity galaxies have lower dust content and lower global infrared emission at fixed $L(24) / L$ (TIR) (Cannon et al. 2005, 2006a, 2006b; Walter et al. 2007). Although some of the low-metallicity galaxies are hotter than the "hottest" high-metallicity galaxies, we do not consider this significant enough in our sample, in light of the broad scatter in properties shown by our low-metallicity galaxies.

The LIRGs still show a deviation, albeit less extreme than in the previous case, from the trend of the other star-forming and starburst galaxies when the ratio $L(24) / L(T I R)$ is plotted as a function of $L(\mathrm{TIR}) / L(3.6)$, i.e., the infrared luminosity per unit stellar mass (Figure 3, right). Thus, the observed deviation is not due to a bias in our choice of the emitting region, but is still present when a different normalization to the infrared luminosity is used. An overestimate of $L$ (TIR) for the LIRGs may potentially produce the observed deviation, but we would need such an overestimate to be in the range $3-100 \times$, in order to reconcile the LIRGs with the other starburst galaxies in Figure 3; this is unlikely to be the case, as our typical uncertainty on $L$ (TIR) is around 25\% (see discussion in Section 3).

We concentrate the rest of the discussion on the highmetallicity sample, to avoid the impact of low dust content on the observed IR luminosity. Figure 4 (left) shows the comparison between data and expectations from models for $L(24) / L(T I R)$ as a function of the ILSD. For clarity, in the left panel of Figure 4 we also show the data after they are binned in 1 dex intervals along the IRSD axis (for two binning schemes shifted by 0.5 dex relative to each other). The models are the same as described in Calzetti et al. (2007), which we summarize briefly here. Stellar populations SEDs are from the Starburst99 models, for a stellar initial mass function (IMF) given by a broken power law as described in Kroupa (2001, see also Chabrier 2003). The dust extinction and geometry are modeled with the empirical prescription of Calzetti (2001), which is accurate for starburst galaxies, but will lose accuracy both at the low end and high end of the SFSD range (Calzetti et al. 1994; Calzetti et al. 2000, 2005; Meurer et al. 1999; Buat et al. 2002; Goldader et al. 2002; Bell 2003; Buat et al. 2005; Seibert et al. 2005). However, we expect, to first approximation, that this simple prescription will at least guide in evaluating trends. The infrared SED is modeled according to the prescription of Draine \& $\mathrm{Li}$ (2007), according to which the fraction of infrared light emerging in each band is a function of the starlight intensity. The starlight intensity in the Draine \& Li (2007) models is related to the SFSD of the stellar population models: larger SFSDs correspond to higher starlight intensity, which in turn produces warmer infrared emission, i.e., higher $L(24) / L$ (TIR) (Calzetti et al. 2007).

Calzetti et al. (2007) model sub-kpc regions in galaxies, thus comparisons with whole galaxies need to be performed with some care. The fiducial models from Calzetti et al. (2007) adopt as default a $100 \mathrm{Myr}$ constant star formation population attenuated by the starburst curve of Calzetti (2001). This requires differential extinction between stars and ionized gas, with $E(B-V)_{\text {star }} \sim 0.44 E(B-V)_{\text {gas }} ;$ furthermore, the models relate the intensity of the SFSD to the opacity of the medium, in the sense that more actively star-forming systems also tend to be more dust-obscured and stronger infrared emitters (Wang \& Heckman 1996; Heckman et al. 1998; Hopkins et al. 2001; Calzetti 2001). While this may be true on average, whole galaxies may encompass a wider range of dust emission characteristics. For instance, in sub-kpc regions or $\mathrm{H}$ II regions larger SFRs (and higher IR emission) imply higher stellar field intensities, which produce hotter infrared SEDs (Draine \& Li 2007). In a galaxy, higher IR emission can also be accomplished with larger filling factors of the infrared-emitting regions; conversely, lower IRSDs do not necessarily need to be accompanied by an increased transparency of the interstellar medium (ISM). Whole galaxies offer a wider range of physical scenarios than $\mathrm{H}$ II regions also in terms of the stellar population mix.

Indeed, we can span a wider range in the parameter space shown in Figure 4 if we add additional simple scenarios to our "default" model. We include, as examples, the cases of (1) constant and large $\left(A_{V}>10 \mathrm{mag}\right)$ dust opacity, independent of the SFSD; (2) $10 \%$ filling factor by area of the SFR relative to the total area considered (e.g., disk galaxies, where star formation is concentrated along the spiral arms, and there is little star formation in the interarm regions); and (3) a variation in the underlying stellar population, where the fiducial $100 \mathrm{Myr}$ old constant star formation population of Calzetti et al. (2007) is accompanied by both $1 \mathrm{Gyr}$ and $10 \mathrm{Gyr}$ constant star formation models and by stellar population models with exponentially decreasing star formation and $e$-folding times of $\tau=2 \mathrm{Gyr}$ and $\tau=5 \mathrm{Gyr}$. We finally add a model in which 

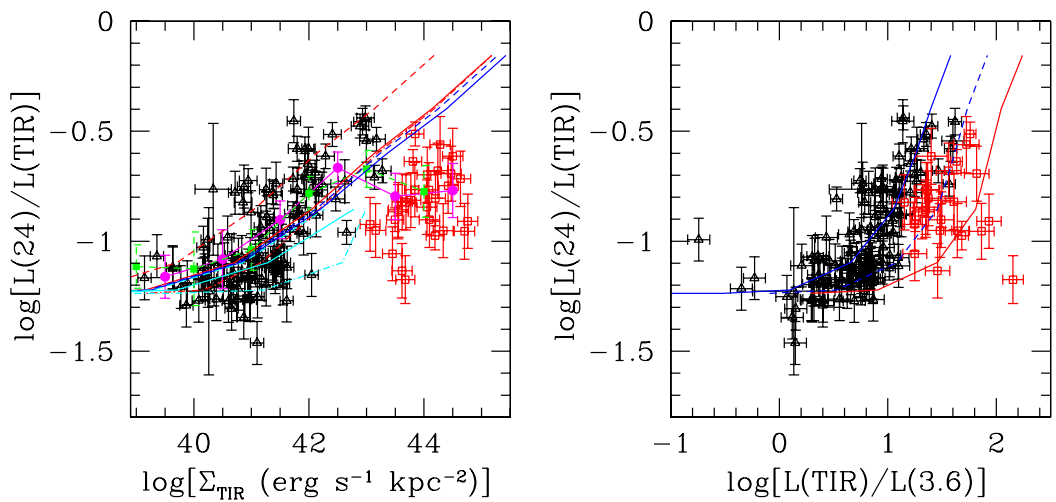

Figure 4. Same as Figure 3 for the high-metallicity galaxies, only. Left: mean values of the data binned in 1 dex intervals are shown in magenta and in green (with $1 \sigma$ error bars), corresponding to binning intervals shifted by 0.5 dex between the magenta and green points. The expected trends for a $100 \mathrm{Myr}$ old, a $1 \mathrm{Gyr}$, and a $10 \mathrm{Gyr}$ constant star formation population reddened by increasing amounts of foreground dust for larger SFRD, with a starburst attenuation curve (default dust model, see Calzetti et al. 2007), are marked by the red continuous, the blue dash, and the blue continuous lines, respectively. The case of exponentially decreasing star formation with $e$-folding times of $\tau=5 \mathrm{Gyr}$ (dashed cyan line) and $2 \mathrm{Gyr}$ (continuous cyan line) are shown for the default dust model. Variations on the default dust model are also shown: a $100 \mathrm{Myr}$ constant star formation population attenuated by constant, large dust opacity $\left(A_{V}>10 \mathrm{mag}\right.$ ) independent of TIR luminosity or SFRD (red dot-dashed line); a $100 \mathrm{Myr}$ constant star formation population attenuated by our default dust model, but with a 10\% filling factor for the IR-emitting regions within the galaxy (red dashed line); and a $\tau=2 \mathrm{Gyr}$ exponentially decreasing star formation model, with the stellar population homogeneously mixed with the dust (cyan dot-dashed line). Right: model lines for a $100 \mathrm{Myr}$ old (red continuous line), a $1 \mathrm{Gyr}$ old (blue dashed line), and a $10 \mathrm{Gyr}$ old (blue continuous line) constant star formation population are overplotted on the data for $L(24) / L(\mathrm{TIR})$ vs. the infrared emission per unit stellar luminosity measured at $3.6 \mu \mathrm{m}$.

the decreasing star formation population with $2 \mathrm{Gyr} e$-folding time is homogeneously mixed with dust; here the extinction curve is adopted to be the mean Milky Way one, with stars and ionized gas affected by the same dust column densities (Calzetti 2001), i.e., no differential extinction is introduced. These scenarios account for much of the observed dispersion of the star-forming and starburst galaxies in Figure 4 (left). For instance, galaxies that have very low $L(24) / L($ TIR) ratios for IRSD > $10^{41} \mathrm{erg} \mathrm{s}^{-1} \mathrm{kpc}^{-2}$ are generally of morphological type Sab or earlier; their infrared SEDs (see, also, Figure 5) are best described by a model of decreasing star formation with the stellar population mixed with the dust (cyan dashed lines in both Figures 4 and 5). In these types of galaxies, in fact, the bolometric emission tends to be dominated by evolved stellar populations; these populations are likely responsible for a non-negligible portion of the infrared luminosity and tend to heat the dust to colder temperatures than actively star-forming populations (e.g., Helou 1986).

The LIRGs tend to have their infrared emission dominated by the current burst of star formation (Scoville et al. 2000), thus by relatively young stellar populations with ages $\lesssim 100 \mathrm{Myr}$. Yet, their $L(24) / L(\mathrm{TIR})$ ratios fall outside of the range spanned by the appropriate models of Figure 4 (left). This ratio is lower than expected for the measured infrared emission even when the latter is normalized to the galaxy's stellar mass (Figure 4, right). Our models (see above) go through the locus occupied by the LIRGs for constant star-forming populations of $\approx 100 \mathrm{Myr}-1 \mathrm{Gyr}$ age. However, this apparent agreement is misleading, since LIRGs have evolved underlying galaxies, and the stellar populations contributing to the $3.6 \mu \mathrm{m}$ emission need to be at least a few $\mathrm{Gyr}$ old (Scoville et al. 2000), requiring a shift by at least a factor of 2 along the horizontal axis of Figure 4, right-hand-side panel.

Supporting evidence that the LIRGs in our sample have cool SEDs, comparable to those of less luminous star-forming/ starburst galaxies, is provided by the dependence of the $L(70) / L$ (TIR) and the $L(160) / L($ TIR) ratios on the IRSD (Figure 5). The models of Draine \& Li (2007), combined with the prescription of Calzetti et al. (2007) to relate the SFSD to the starlight intensity, predict that by the time the IRSD of LIRGs is reached, the peak of the IR SED should have moved out of the MIPS $70 \mu \mathrm{m}$ band, toward shorter wavelengths, thus following first an increase in the $L(70) / L$ (TIR) ratio from low luminosities, to a peak around $\Sigma_{\text {TIR }} \approx 10^{42} \mathrm{erg} \mathrm{s}^{-1} \mathrm{kpc}^{-2}$, to be followed by a steady decrease. Similarly, the same models predict a steady decrease of the contribution of the $160 \mu \mathrm{m}$ emission to the total far-infrared luminosity as the peak emission moves to shorter wavelengths at higher luminosity. The data are, instead, consistent with a roughly constant $L(70) / L(\mathrm{TIR})$ over, at least, 4 orders of magnitude from $\Sigma_{\mathrm{TIR}} \sim 10^{41}-10^{45} \mathrm{erg} \mathrm{s}^{-1} \mathrm{kpc}^{-2}$, and possibly over the full range covered by our data. The trend and dispersion of the data at low IRSD $\left(\Sigma_{\mathrm{TIR}}<10^{42} \mathrm{erg} \mathrm{s}^{-1} \mathrm{kpc}^{-2}\right)$ can be, at least partially, accounted for by the model scenarios discussed above. The high-luminosity trend, on the other hand, cannot be accounted for by any of our simple models. A similar argument can be applied to the $L(160) / L$ (TIR) ratio (Figure 5, right), which, after a decrease reasonably consistent with the models for increasing luminosity up to $\Sigma_{\mathrm{TIR}} \sim 10^{42.5} \mathrm{erg} \mathrm{s}^{-1} \mathrm{kpc}^{-2}$, flattens out for the highest values of the IRSD, indicating the presence of an extra contribution from cool dust to the infrared SED. When combined with the findings for $L(24) / L$ (TIR) (Figure 4), we infer that, for the LIRGs, the energy absorbed by the dust itself in the mid-IR (self-absorption; Rieke et al. 2009) is re-emitted at longer infrared wavelengths, thus producing the cool IR SEDs observed by Dunne \& Eales (2001). For the purpose of this work, we will modify the models of Draine \& Li (2007), by imposing that $L(70) / L(\mathrm{TIR})=\mathrm{constant}=0.5$ and $L(160) / L(\mathrm{TIR})$ $\sim$ constant $=0.21$ for $\Sigma_{\mathrm{TIR}}>10^{42.5} \mathrm{erg} \mathrm{s}^{-1} \mathrm{kpc}^{-2}$ (indicated by the black lines in both panels of Figure 5).

The overall conclusion from the above discussion is that normal star-forming and starburst galaxies tend to have "hotter" dust, i.e., increasing $L(24) / L(T I R)$ ratios, for increasing IRSD, in agreement with the predictions of Draine \& $\mathrm{Li}$ (2007) coupled with a direct correlation between SFR and stellar field intensity (Calzetti et al. 2007). However, the trend "flattens" around an IRSD $\sim 0.3-1 \times 10^{43} \mathrm{erg} \mathrm{s}^{-1} \mathrm{kpc}^{-2}$ (Figure 4, left). This roughly corresponds to $L(\mathrm{TIR}) \approx 1-3 \times 10^{44} \mathrm{erg} \mathrm{s}^{-1} \sim 3-8 \times 10^{10} L_{\odot}$ or $L(24) \approx 2-6 \times 10^{43} \mathrm{erg} \mathrm{s}^{-1} \sim 0.6-2 \times 10^{10} L_{\odot}$. The conversion between luminosity/area and luminosity should, however, be taken with caution, as it carries a large scatter, almost an order of magnitude. Galaxies with IRSDs or luminosities above these values, which in our sample include mostly LIRGs, show some evidence for flattening or decreasing $L(24) / L(\mathrm{TIR})$ 

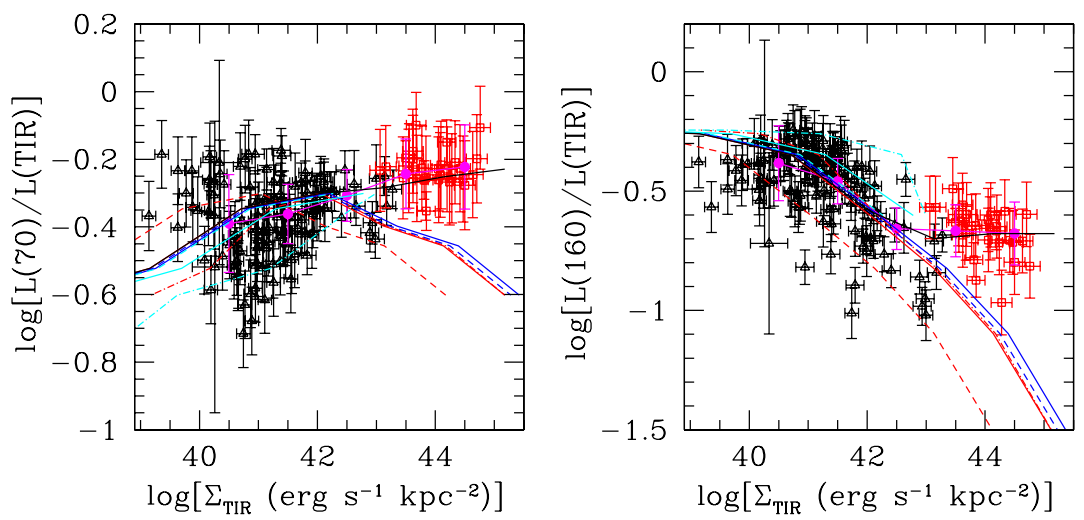

Figure 5. $70 \mu \mathrm{m}$-to-TIR and the $160 \mu \mathrm{m}$-to-TIR ratios as a function of the IRSD for the metal-rich galaxies in our sample. As in Figure 4, the LIRGs are marked with red symbols. Mean values of the data binned in intervals of 1 dex each are shown in magenta. The model lines are the same as in Figure 4 (left). According to those models, the peak of the infrared emission moves in and out of the $70 \mu \mathrm{m}$ band, i.e., the IR SED becomes of sufficiently high effective temperature that the peak IR emission moves to shorter wavelengths than the MIPS $70 \mu \mathrm{m}$ band, for the range of luminosity surface densities (LSDs) spanned by our sample (Draine \& Li 2007, see their Figure 15). For the same models, the $160 \mu \mathrm{m}$ emission becomes progressively fainter, as the IRSD increases. Modified models for both $L(70) / L(\mathrm{TIR})$ and $L(160) / L(T I R)$ that account for the observed properties of the luminous galaxies, and the LIRGs in particular, require those luminosity ratios to remain approximately constant beyond IRSD $\Sigma_{\mathrm{TIR}} \approx 10^{42-42.5} \mathrm{erg} \mathrm{s}^{-1} \mathrm{kpc}^{-2}$ (black line).

and flattening $L(70) / L(\mathrm{TIR})$ and $L(160) / L(\mathrm{TIR})$ values with increasing IRSD, corresponding to "cooler" dust emission than expected from the intensity of the infrared emission (Dunne et al. 2000; Dunne \& Eales 2001). This trend is likely a manifestation of self-absorption by dust even at $24 \mu \mathrm{m}$ (Rieke et al. 2009, see Section 7 for a quantification of this effect). An inflection in the $L(24) / L\left(\right.$ TIR) trend above $L($ TIR $) \sim 10{ }^{11} L_{\odot}$ has indeed been previously observed by Rieke et al. (2009, see their Figure 8). As suggested by these authors, the presence of self-absorption even at $24 \mu \mathrm{m}$ in LIRGs will hamper the usefulness of standard SFR indicators based on optical-infrared hydrogen recombination lines at these high-luminosity values (see below).

\subsection{Comparisons Between Calibrations}

Several calibrations of SFRs using the $24 \mu \mathrm{m}$ emission of galaxies (or star-forming regions in galaxies) have been recently published, using both simple proportionality between SFR and $L(24)$ and nonlinear relations. The calibrations are based on a variety of galaxy and/or region samples, and stellar IMFs. For sake of comparison among the different calibrations, we report below most of the published ones, all of them converted to a common IMF and luminosity scale. In this section, we will use luminosity, rather than our preferred (because distance- and sizeindependent) luminosity/area, since most published calibrations are derived from luminosities. We choose the Kroupa (Kroupa 2001) IMF, which has a slope -2.3 for stellar masses in the range $0.5-100 M_{\odot}$ and -1.3 for stellar masses in the range 0.1-0.5 $M_{\odot}$. For this IMF, Starburst99 stellar population models with solar metallicity and constant SFR give the following relation between number of ionizing photons and SFR:

$$
\operatorname{SFR}\left(M_{\odot} \mathrm{yr}^{-1}\right)=7.41 \times 10^{-54} N_{\text {ion }}\left(\mathrm{s}^{-1}\right),
$$

which corresponds to ${ }^{22}$

$$
\mathrm{SFR}(\mathrm{H} \alpha)\left(M_{\odot} \mathrm{yr}^{-1}\right)=5.45 \times 10^{-42} L(\mathrm{H} \alpha)\left(\mathrm{erg} \mathrm{s}^{-1}\right),
$$

22 The calibration coefficient in Equation (5) is about 3\% higher than that reported in Calzetti et al. (2007, their Equation (6)). The present calibration is based on a $>1 \mathrm{Gyr}$ age constant star formation stellar population, while the calibration in Calzetti et al. (2007) is based on a $100 \mathrm{Myr}$ age constant star formation population. This difference is, however, smaller than the $\sim 12 \%$ uncertainty to be expected for variations in the physical conditions of the emitting regions, i.e., variations in electron temperature between $5000 \mathrm{~K}$ and $15,000 \mathrm{~K}$, consistent with the variations in metallicity typical of our galaxy sample (Osterbrock \& Ferland 2006). where the $\mathrm{H} \alpha$ luminosity is extinction corrected. For reference, adopting a Salpeter (1955) IMF in the stellar mass range $0.1-100 M_{\odot}$ would increase the calibration coefficient in Equation (5) by a factor 1.51.

Linear relations between SFR and $L(24)$ have been published by Wu et al. (2005), Zhu et al. (2008), and Rieke et al. (2009). Reported on a common scale, the three relations ${ }^{23}$ are

$$
\begin{aligned}
\operatorname{SFR}(24)_{W 05, l}= & 2.75 \times 10^{-43} L(24) \\
& 1 \times 10^{42} \lesssim L(24) \lesssim 1 \times 10^{44},
\end{aligned}
$$

$$
\begin{aligned}
\operatorname{SFR}(24)_{Z 08, l}= & 2.46 \times 10^{-43} L(24) \\
& 4 \times 10^{41} \lesssim L(24) \lesssim 2 \times 10^{44},
\end{aligned}
$$

$$
\begin{aligned}
\operatorname{SFR}(24)_{R 09}= & 2.04 \times 10^{-43} L(24), \\
& 4 \times 10^{42} \leqslant L(24) \leqslant 5 \times 10^{43}, \\
= & 2.04 \times 10^{-43} L(24) \times\left[2.03 \times 10^{-44} L(24)\right]^{0.048} \\
& L(24)>5 \times 10^{43},
\end{aligned}
$$

where the $24 \mu \mathrm{m}$ luminosities are in units of erg s${ }^{-1}$. The linear (with a small nonlinear correction at the high luminosities for the derivation of Rieke et al. 2009) relations are averages of the calibrations derived in each paper. The galaxy samples and the reference calibrators are different in the three papers: $\mathrm{Wu}$ et al. (2005) use both radio and the extinction-corrected $\mathrm{H} \alpha$ luminosity of the galaxies in common between the SDSS and the Spitzer First Look Survey sample; Zhu et al. (2008) use extinction-corrected UV and $\mathrm{H} \alpha$ luminosities and FIR emission of the galaxies in the SDSS and the Spitzer SWIRE galaxy sample (Lonsdale et al. 2003); Rieke et al. (2009) use both $\mathrm{P} \alpha$ and FIR emission on templates of LIRGs and ULIRGs with data of various origins. Despite these differences, the three calibrations are remarkably close, within $\sim 10 \%-35 \%$ of each other, consistent with the calibration uncertainities of $0.1-0.15$ dex quoted by the authors.

\footnotetext{
23 The luminosity range of applicability for the calibrations by $\mathrm{Wu}$ et al. (2005) and Zhu et al. (2008) is not explicitly provided by the authors. We report ranges as derived from the luminosity range of the main samples from
} those authors. 
Linear calibrations are derived under the assumption that the $24 \mu \mathrm{m}$ luminosity increases proportionally to the SFR. The alternative scenario discussed in the previous section predicts the $24 \mu \mathrm{m}$ luminosity to increase proportionally faster than the SFR, as a result of the increasing mean dust temperature, and corresponding shift to bluer wavelengths of the infrared SED. This trend may simply imply, for whole galaxies, a progressive shift for the dominant population heating the dust from evolved stars to young stellar populations; this argument is expected to hold on average, rather than on a galaxy-by-galaxy basis. As seen in Section 4.1, the effective dust temperature appears to reach a plateau as a function of increasing TIR luminosity around $L(24) \sim$ a few $\times 10^{43} \mathrm{erg} \mathrm{s}^{-1}$.

Direct fits of $L(24)$ as a function of a variety of reference SFR indicators do show such nonlinear behavior $(\mathrm{Wu}$ et al. 2005; Alonso-Herrero et al. 2006; Perez-Gonzalez et al. 2006; Calzetti et al. 2007; Zhu et al. 2008), and the relation between SFR and $L(24)$ requires the mediation of a power law with lessthan-unity values. Two of the published calibrations involving whole galaxies are from Wu et al. (2005) and Zhu et al. (2008), which expressed in terms of our default IMF become

$$
\begin{aligned}
\operatorname{SFR}(24)_{W 05}= & 1.07 \times 10^{-38} L(24)^{0.893} \\
& 1 \times 10^{42} \lesssim L(24) \lesssim 1 \times 10^{44}, \\
\operatorname{SFR}(24)_{Z 08}= & 8.10 \times 10^{-37} L(24)^{0.848} \\
& 4 \times 10^{41} \lesssim L(24) \lesssim 2 \times 10^{44},
\end{aligned}
$$

where the units are the same as the previous linear equations. Calibrations involving either $\mathrm{H}$ II-emitting regions in galaxies (Perez-Gonzalez et al. 2006; Calzetti et al. 2007; Relaño et al. 2007) or combinations of $\mathrm{H}$ II regions and LIRGs (AlonsoHerrero et al. 2006) (called "H II regions" calibrations from now on) are given by ${ }^{24}$

$$
\begin{aligned}
\operatorname{SFR}(24)_{P 06}= & 9.01 \times 10^{-34} L(24)^{0.768} \\
& 1 \times 10^{38} \lesssim L(24) \lesssim 3 \times 10^{41}, \\
\operatorname{SFR}(24)_{A 06}= & 5.83 \times 10^{-38} L(24)^{0.871} \\
& 1 \times 10^{40} \lesssim L(24) \lesssim 3 \times 10^{44}, \\
\operatorname{SFR}(24)_{\operatorname{C} 07}= & 1.31 \times 10^{-38} L(24)^{0.885} \\
& 1 \times 10^{40} \lesssim L(24) \lesssim 3 \times 10^{44}, \\
\operatorname{SFR}(24)_{\operatorname{Re} 07}= & 5.66 \times 10^{-36} L(24)^{0.826} \\
& 1 \times 10^{38} \lesssim L(24) \lesssim 3 \times 10^{44} .
\end{aligned}
$$

Typical uncertainties for the calibrations of Equations (9)-(14) are $0.01-0.02$ in the exponent $(\sim 0.06$ for Wu et al. 2005$))$ and $6 \%-15 \%$ in the calibration constant. Within these uncertainties, and within the typical scatter of $0.2-0.3$ dex of galaxy and $\mathrm{H}$ II regions samples, all calibrations mostly agree with each other, when limited to the luminosity ranges where they were derived. Deviations from one to the other are around a factor

\footnotetext{
24 As in the case of Wu et al. (2005) and Zhu et al. (2008), most authors do not explicitly provide the luminosity range of validity of their SFR calibrations. As before, we report limits derived from the luminosity range of the main samples from each paper.
}

$\sim 2$ (Figure 6, left panel). However, customary use does not carry the uncertainties for the SFR calibrations; this can produce larger discrepancies than a factor $\sim 2$ when the calibrations are extrapolated beyond their range of validity, as exemplified by the left panel of Figure 6.

Galaxy-wide SFR(24) nonlinear calibrations (Wu et al. 2005; Zhu et al. 2008) are marginally, 25\%-60\%, albeit systematically, higher than $\mathrm{H}$ II region SFR(24) calibrations at the highluminosity end (Figure 6, left panel). The same is observed for galaxy-wide linear calibrations (Equations (6)-(8), and Figure 6 , right panel), with deviations around a factor $\sim 2$ relative to $\mathrm{H}$ II region nonlinear calibrations, which is, again, systematic, albeit marginal within the uncertainties. In order to account for this difference, we can speculate that, in whole galaxies, the contrast between the contributions of recent star formation and old stellar populations in the heating of dust is lower than in their $\mathrm{H}$ II regions. This possibly leads to a lower average dust temperature (and lower, on average, $L(24) / L(\mathrm{TIR})$ ) and, consequently, to a higher calibration constant for $\operatorname{SFR}(24)$. However, this speculation cannot be easily verified at this time; it will have to await for observations with the Herschel Space Telescope, in order to obtain long infrared wavelength images of sufficient high angular resolution to isolate $\mathrm{H}$ II-emissiondominated regions in external galaxies.

The comparison of the linear SFR(24) calibrations (Equations (6)-(8), all of them for whole galaxies) with the nonlinear ones (Figure 6, right, compared with the nonlinear calibration of Relaño et al. 2007) shows, again, general agreement within the existing uncertainties, when considered in the luminosity range for which those calibrations were derived. However, extending the linear calibrations to the lowest luminosity values of our high-metallicity sample show significant discrepancies from the nonlinear calibrations; specifically, the linear calibrations predict SFRs that are a factor $\sim 2-4$ lower than predicted by the nonlinear calibrations. This is expected in light of the fact that galaxies become increasingly transparent for decreasing infrared luminosity, and a larger fraction of the light from recent star formation emerges directly at UV and optical wavelengths. Thus, the linear SFR(24) calibrations will underestimate the true SFR below $L(24) \approx 3 \times 10^{42} \mathrm{erg} \mathrm{s}^{-1}$ (Figure 6, right).

Nonlinear calibrations may provide a better alternative for extending the SFR(24) to low-luminosity values, because their functional form accounts for two physical effects: increasing transparency of the medium and lower effective dust temperature toward lower SFSDs (Draine \& Li 2007). However, they are also potentially problematic. The first problem is that for sufficiently low dust content, even the nonlinear SFR(24) will end up underestimating the true SFR (Calzetti et al. 2007). The second problem is that low global SFRs can be obtained both for less active, metal-rich (but gas-poor) galaxies and for active, low-metallicity, low-luminosity galaxies. In the latter case, as the metal content of the system decreases, the effective dust temperature increases (e.g., Hunter et al. 1989; Walter et al. 2007; Engelbracht et al. 2008, and Figure 4), thus complicating any trend. A third problem is that nonlinear calibrations between SFR and luminosity are "object-dependent," in the sense that their validity relies on the presence of the physical conditions that lead to the nonlinear relation itself. Thus, calibrations obtained for whole galaxies will in general not be applicable to regions within those galaxies, and vice versa.

Indeed, the decreasing dust opacity of galaxies for decreasing SFR (Wang \& Heckman 1996; Calzetti et al. 2007) calls into 

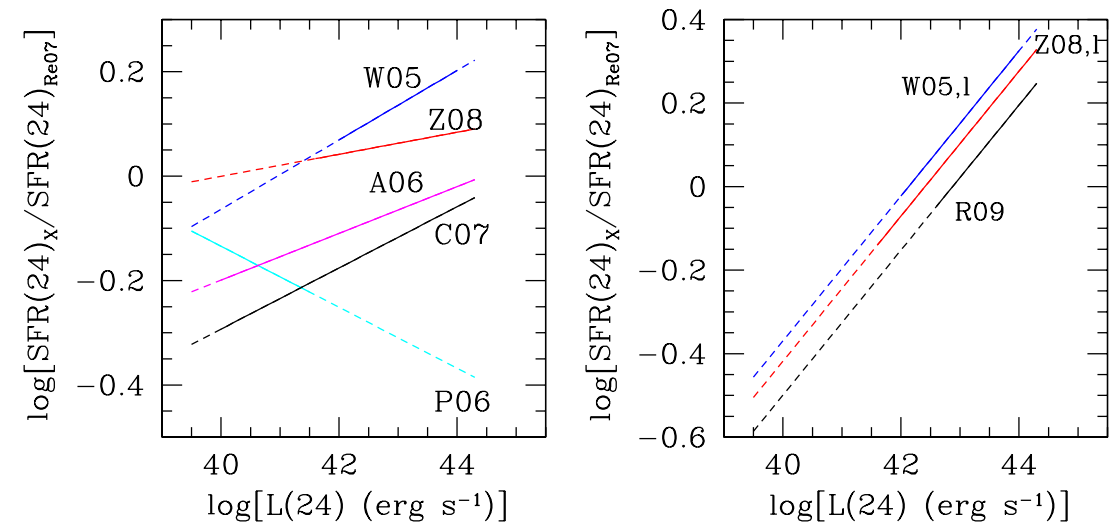

Figure 6. Ratio of SFR calibrations using the $24 \mu \mathrm{m}$ luminosity, as a function of the observed $L(24)$ (in $\mathrm{erg} \mathrm{s}^{-1}$ ), for the luminosity range spanned by our high-metallicity galaxies sample (excluding LIRGs), $39.5 \lesssim \log [L(24)] \lesssim 44.3$. Left: the ratio of the nonlinear calibrations of Wu et al. (2005, W05, blue line), Alonso-Herrero et al. (2006, A06, magenta line), Perez-Gonzalez et al. (2006, P06, cyan line), Calzetti et al. (2007, C07, black line), and Zhu et al. (2008, Z08, red line) to the nonlinear calibration of Relaño et al. (2007). The continuous lines show the luminosity range used by the authors to derive their calibrations, while the dashed lines extend those calibrations to the entire luminosity range spanned by our high-metallicity sample. The nonlinear calibration of Relaño et al. (2007) is used as reference because it has been derived for the entire luminosity range displayed in our figure (see Equation (14)). Right: the ratio of the linear calibrations of Wu et al. (2005, Wu05,1, blue line), Zhu et al. (2008, Z08,1, red line), and Rieke et al. (2009, R09, black line) to the nonlinear calibration of Relaño et al. (2007). As in the left panel, continuous and dashed lines indicate range of derivation and extrapolation of the calibrations, respectively.

(A color version of this figure is available in the online journal.)

question the usefulness of an infrared-based SFR indicator at the low-luminosity end of our sample, especially in the lowmetallicity regime. For many of the galaxies in our sample star formation appears to "shine" unimpeded by dust absorption. This is evidenced in Figure 7, where the ratio of $\mathrm{H} \alpha$ to the $24 \mu \mathrm{m}$ luminosity (rescaled according to the proportionality factor of Kennicutt et al. 2009, between the two luminosities) is plotted as a function of the SFR as defined in Kennicutt et al. (2009) for the normal star-forming and starburst galaxies in our sample. For most of these galaxies, the amount of star formation emerging at optical wavelengths (as traced by $\mathrm{H} \alpha$ ) is larger than the star formation emerging in the infrared (as traced by the $24 \mu \mathrm{m}$ emission). For this reason, composite SFR indicators, involving the combination of an optical and an infrared tracer of SFR, have been proposed (Calzetti et al. 2007; Kennicutt et al. 2007, 2009; Zhu et al. 2008). For whole galaxies, Kennicutt et al. (2009) propose (see, also Zhu et al. 2008)

$$
\begin{aligned}
\mathrm{SFR}_{\text {mix }, \mathrm{K} 09=} & 5.45 \times 10^{-42}\left[L(\mathrm{H} \alpha)_{\mathrm{obs}}+0.020 L(24)\right] \\
& 3 \times 10^{38} \lesssim L(24) \lesssim 3 \times 10^{44},
\end{aligned}
$$

where the calibration is expressed in terms of our selected IMF. For H II-dominated regions and starburst galaxies, Calzetti et al. (2007) propose

$$
\begin{aligned}
\mathrm{SFR}_{\text {mix }, \mathrm{C} 07}= & 5.45 \times 10^{-42}\left[L(\mathrm{H} \alpha)_{\mathrm{obs}}+0.031 L(24)\right] \\
& 3 \times 10^{38} \lesssim L(24) \lesssim 3 \times 10^{44} .
\end{aligned}
$$

In both equations, $L(\mathrm{H} \alpha)_{\text {obs }}$ is the observed (non-extinctioncorrected) luminosity at $\mathrm{H} \alpha$. The difference between the two equations has been suggested to be due to a difference in the underlying stellar population heating the dust: continuous star formation no older than about $100 \mathrm{Myr}$ for starburst galaxies (and instantaneous bursts only a few Myr old for H II regions) and much longer lived star formation, of order $10 \mathrm{Gyr}$, for whole galaxies (Kennicutt et al. 2009).

Our sample spans about 6 orders of magnitude in SFRs, from $\sim 10^{-4} M_{\odot} \mathrm{yr}^{-1}$ all the way up to LIRGs producing stars at a pace close to a hundred $M_{\odot} \mathrm{yr}^{-1}$. The stellar populations dominating the dust heating will thus transition from those characteristic of normal star-forming spirals or irregulars (with timescales

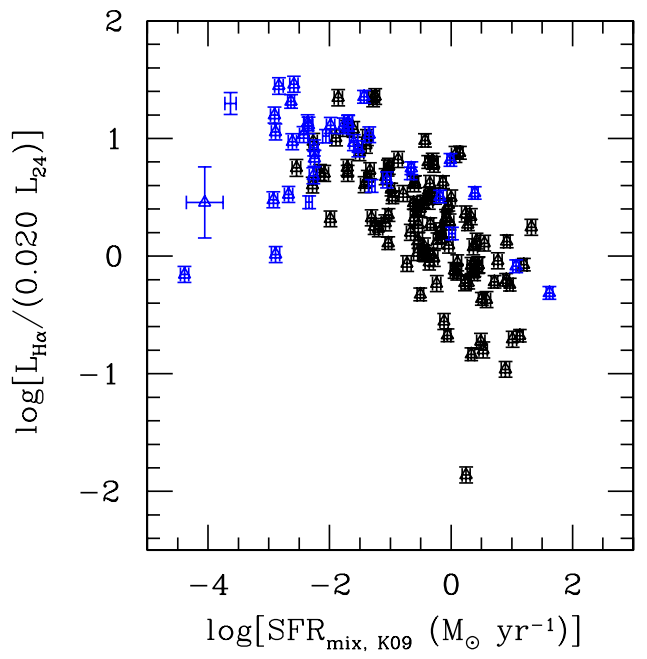

Figure 7. Ratio between the $\mathrm{H} \alpha$ luminosity and the scaled $24 \mu \mathrm{m}$ luminosity for the galaxies in our sample (excluding LIRGs) as a function of SFR (Equation (15)). Blue points mark low-metallicity galaxies, and black points the high-metallicity ones. The scaling factor for the $24 \mu \mathrm{m}$ luminosity used on the vertical axis of this figure is from Equation (15) (Kennicutt et al. 2009). This luminosity ratio measures the fraction of the star formation that emerges from the galaxy unattenuated by dust. Not surprisingly, there is a correlation between this luminosity ratio and SFR, in the sense that more actively starforming galaxies are fainter in the optical relative to the infrared emission (see, also, Calzetti et al. 2007). In our normal star-forming and starburst sample, most of the galaxies tend to be relatively transparent, meaning that a large fraction of the light from recent star formation emerges unabsorbed by dust.

(A color version of this figure is available in the online journal.)

for star formation of order $10 \mathrm{Gyr}$ ) to those characteristic of young starbursts (timescales of order $100 \mathrm{Myr}$ or less). In particular, we expect that as we approach the luminosity of the LIRGs, and consequently high dust opacities in the galaxies, the inferred SFRs will need to coincide with those derived from SFR(24). In Figure 8, we compare both Equations (15) and (16) among themselves and with the SFR(24) calibration of Rieke et al. (2009), for the galaxies in our sample (excluding LIRGs). The difference between Equations (15) and (16) is typically less than $\sim 15 \%-20 \%$ up to $L(24) \sim 4 \times 10^{42} \mathrm{erg} \mathrm{s}^{-1}$, and be- 


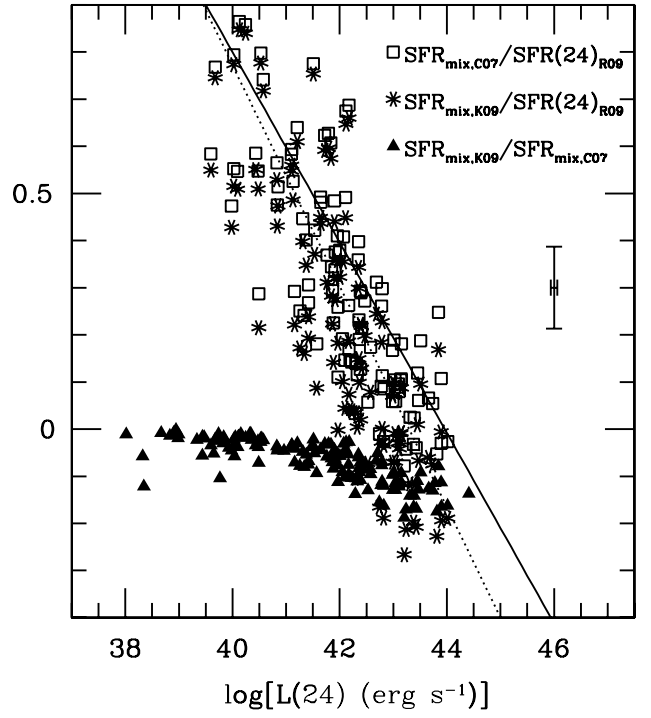

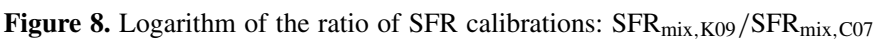

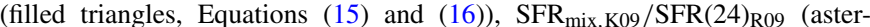

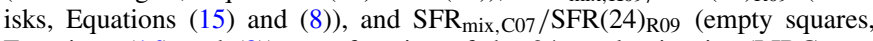
Equations (16) and (8)) as a function of the $24 \mu \mathrm{m}$ luminosity (LIRGs are excluded). Fits to the data from the latter two ratios are shown. The calibration SFR $(24)_{\mathrm{R} 09}$ is extended below its limit of validity, $\log [L(24)] \sim 42.6$, for illustrative purposes only. A typical $1 \sigma$ error bar is shown as well.

comes larger for luminosities above that value. However, this is also approaching the luminosity range where $\mathrm{SFR}_{\text {mix }}$ needs to reach, as asymptotic value, $\operatorname{SFR}(24)$, as galaxies become more and more dominated by infrared emission. We elect to use Equation (15) to derive SFRs for galaxies with luminosity $L(24)$ $\lesssim 4 \times 10^{42} \mathrm{erg} \mathrm{s}^{-1}$, and Equation (16) for higher luminosity; this "transition" luminosity corresponds to SFR $\sim 1 M_{\odot} \mathrm{yr}^{-1}$, which is a transition point where SFRs are equally contributed by the $\mathrm{H} \alpha$ and $24 \mu \mathrm{m}$ luminosities (Figure 7). In our sample, that transition SFR corresponds to $\Sigma_{\mathrm{SFR}} \approx 0.05 \mathrm{M}_{\odot} \mathrm{yr}^{-1} \mathrm{kpc}^{-2}$, albeit with a large uncertainty, since the relation between SFR and $\Sigma_{\mathrm{SFR}}$ has over 1 order of magnitude scatter. The exact value of the transition point is not crucial, as the difference between SFR values (Equations (15) and (16)) around that point is $\sim 25 \%$ $(\sim 0.1$ dex in logarithmic scale), and physical systems are expected to transition from star formation timescales of many $\mathrm{Gyr}$ down to a few hundred Myr in a smoother way than what we describe here.

Equation (16) is almost identical to the linear part of Equation (8) for $L(\mathrm{H} \alpha)_{\text {obs }} \ll 0.031 L(24)$. The closeness of the two equations becomes even more obvious if we recall that we measure a mean ratio $L(\mathrm{H} \alpha) / L(24)=0.031$ for the metal-rich regions/galaxies in the sample of Calzetti et al. (2007), rather than 0.038, as measured by Rieke et al. (2009) in the same luminosity range $10^{7} L_{\odot} \leqslant L(24) \leqslant 10^{10} L_{\odot}$. The difference is small, about $20 \%$. The ratio $L(\mathrm{H} \alpha) / L(24)=0.031$, when combined with the median ratio $L(24) / L($ TIR) $\sim 0.16$ (see Figure 3, and also Rieke et al. 2009) for the LIRGs, yields a mean value for $L(\mathrm{H} \alpha) / L(\mathrm{TIR}) \sim 0.005$, consistent with expectations for a $100 \mathrm{Myr}$ old constant star formation population (from the models of Leitherer et al. 1999, where we assume $L$ (TIR) $\sim L$ (bol), the bolometric luminosity). It is worth noticing that the ratio $L(\mathrm{H} \alpha) / L(24)=0.038$ determined by Rieke et al. (2009) yields values for $L(\mathrm{H} \alpha) / L(\mathrm{TIR}) \sim 0.006$, consistent with a $30 \mathrm{Myr}$ old constant star formation population. Both calibrations are, thus, consistent with infrared emission from galaxies whose emission is dominated by the young stellar population component, as expected for starbursts and LIRGs (e.g., Scoville et al. 2000, and references therein). We use our own measured value of the $L(\mathrm{H} \alpha) / L(24)$ mean ratio, and we replace it in Equation (8), to derive SFRs in the high-luminosity range of our sample.

The lowest luminosity LIRG in our sample has $L(24) \sim$ $4 \times 10^{43} \mathrm{erg} \mathrm{s}^{-1}$, and the relative contributions of $L(24)$ and $L(\mathrm{H} \alpha)_{\text {obs }}$ to the SFR now weight in favor of $L(24)$, with $L(\mathrm{H} \alpha)_{\text {obs }} /(0.031 L(24))<0.14$. Thus, for our purposes, we can ignore the contribution of $L(\mathrm{H} \alpha)_{\mathrm{obs}}$ to the SFR at the highluminosity end, and we will use the re-normalized Equation (8) to measure SFRs for the LIRGs in our sample. Of the two expressions in Equation (8), we only use the nonlinear part, in agreement with the prescription of Rieke et al. (2009) for our range of luminosities. The nonlinear correction in Equation (8) attempts to correct for the presence of self-absorption in the LIRGs and brighter galaxies (Rieke et al. 2009). Derivations of SFR(24) for whole galaxies by other authors do not include such nonlinear terms although their luminosity range often includes LIRGs (Equations (6), (7), (9), (10), and (12)); the presence of a nonlinear term can remain unnoticed if optical or infrared hydrogen recombination lines $(\mathrm{H} \alpha, \mathrm{P} \alpha)$ are used as reference SFR indicators, since, even when extinction corrected, the luminosity of those lines will be underestimated in the presence of high dust opacity (as typical of LIRGs or brighter galaxies: $L(24)>$ a few $10^{43} \mathrm{erg} \mathrm{s}^{-1}$ ). We note that in our sample the highest luminosity LIRG $\left(L(24) \sim 8.3 \times 10^{44} \mathrm{erg} \mathrm{s}^{-1}\right.$ ) shows a difference between the SFRs derived from the re-normalized Equation (8) and its linear version, Equation (16), by less than $15 \%$; for 27 of 29 LIRGs, this difference is less than $10 \%$.

In summary, we derive SFRs for our sample of galaxies as follows:

$$
\begin{aligned}
\mathrm{SFR}= & 5.45 \times 10^{-42}\left[L(\mathrm{H} \alpha)_{\mathrm{obs}}+0.020 L(24)\right] \\
& L(24)<4 \times 10^{42}, \\
= & 5.45 \times 10^{-42}\left[L(\mathrm{H} \alpha)_{\mathrm{obs}}+0.031 L(24)\right] \\
& 4 \times 10^{42} \leqslant L(24)<5 \times 10^{43} \\
= & 1.70 \times 10^{-43} L(24) \times\left[2.03 \times 10^{-44} L(24)\right]^{0.048} \\
& L(24) \geqslant 5 \times 10^{43}
\end{aligned}
$$

where the SFR is in units of $M_{\odot} \mathrm{yr}^{-1}$, and luminosities are in units of $\mathrm{erg} \mathrm{s}^{-1}$. The transition regions between the three regimes are not sharp, and especially the one between Equations (15) and (16) may begin at lower luminosity than the one used here. However, differences between the average SFRs at the transition points are about or less than $0.1 \mathrm{dex}$, which is smaller than both our typical error bar and the spread in the data points.

Equation (17) attempts to capture a general behavior of galaxies in regard to their dust opacity. Below $L(24) \sim 4.0 \times$ $10^{42} \mathrm{erg} \mathrm{s}^{-1}\left(L(\mathrm{TIR}) \approx 10^{10} L_{\odot}\right)$, the young, star-forming regions in galaxies become increasingly transparent at optical and UV wavelengths for decreasing infrared luminosity (Buat et al. 2007, e.g.), and optical emission lines are useful tools for measuring their dust opacity (Kennicutt et al. 2009). At higher infrared luminosities, the star-forming regions become on average increasingly opaque, and, above $L(24) \sim 5 \times$ $10^{43}\left(L(\mathrm{TIR}) \approx 10^{11} L_{\odot}\right)$, their infrared emission becomes the most reliable SFR indicator (Rieke et al. 2009, e.g.). In between $L(\mathrm{TIR}) \approx 10^{10} L_{\odot}$ and $L(\mathrm{TIR}) \approx 10^{11} L_{\odot}$ is a transition regime, where the SFR traced by optical and infrared line emission (e.g., $\mathrm{P} \alpha$ ) is still non-negligible (Calzetti et al. 2007), although it becomes vanishingly small as the upper luminosity limit is reached. This argument should be interpreted 

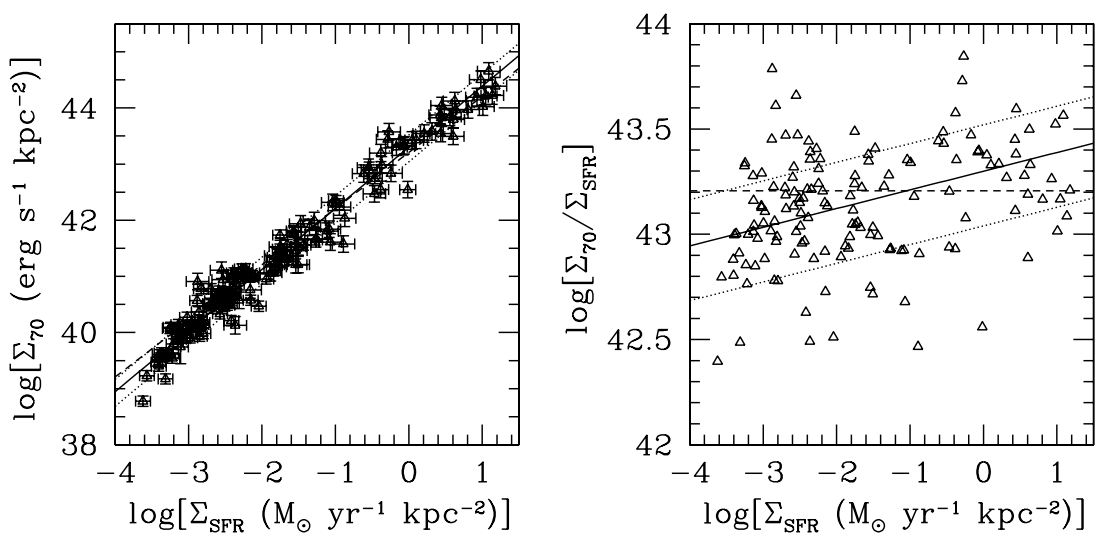

Figure 9. Left: the $70 \mu \mathrm{m} \mathrm{LSD}, \Sigma_{70}=L(70) /$ area, as a function of the SFSD, $\Sigma_{\mathrm{SFR}}$, in log-log scale. In both cases, the area used is the H $\alpha$ emitting area (Figure 2). Only the high-metallicity galaxies are reported (142 data points, with their $1 \sigma$ error bars), together with the best fit (continuous line) and the $1 \sigma$ envelope to the data (dotted lines, which correspond to the range $-0.26,+0.22 \mathrm{dex}$ ). The $1 \sigma$ envelope is calculated as the standard deviation of the logarithm of the ratio relative to the mean trend. The best fit with unity slope is shown as a dashed line. Right: the same data and best-fit lines are shown now for the ratio $\Sigma_{70} / \Sigma_{\mathrm{SFR}}$ as a function of the SFSD. Error bars are omitted for clarity. This plot better shows the deviation of the data points from a one-to-one relation.

as "mean behavior," as galaxies generally contain large numbers of star-forming regions, with different levels of dust opacity. For instance, many galaxies with $L(24)>5 \times 10^{43}$ still show measurable emission at blue wavelengths, specifically $\mathrm{H} \beta(\lambda 4861 \AA$ ) (Kennicutt et al. 2009).

For the 10 starburst galaxies with $\mathrm{P} \alpha$ data, we use this emission line, after correction for dust extinction, to measure the SFRs, adopting a ratio $\mathrm{H} \alpha / \mathrm{P} \alpha=7.82$ for the highmetallicity galaxies and $\mathrm{H} \alpha / \mathrm{P} \alpha=8.73$ for the low-metallicity ones (Calzetti et al. 2007).

\section{CALIBRATION OF SFR(70)}

In order to establish whether the $70 \mu \mathrm{m}$ luminosity can be used as an SFR indicator, and to check its limitations, we first concentrate on the 142 galaxies that constitute the highmetallicity sample. We will discuss the effect of metallicity on our calibrations later in this section.

The $70 \mu \mathrm{m}$ LSD tightly correlates with the SFSD (Figure 9) over the full 5 orders of magnitude spanned by our high-metallicity sample. The best fit through the data points is

$$
\begin{aligned}
\log \left[\Sigma_{70}\left(\mathrm{erg} \mathrm{s}^{-1} \mathrm{kpc}^{-2}\right)\right]= & (1.089 \pm 0.013) \\
& \times \log \left[\Sigma_{\mathrm{SFR}}\left(M_{\odot} \mathrm{yr}^{-1} \mathrm{kpc}^{-2}\right)\right] \\
& +(43.303 \pm 0.029),
\end{aligned}
$$

with a $1 \sigma$ dispersion of the data around the best-fit line of $(-0.26,+0.22)$ dex. Although the best fit is nonlinear, we need to recall that data with lower SFSD often correspond to galaxies with star formation scattered across the disk. These systems generally tend to be less dust opaque than systems with large and concentrated SFRs (Figures 4 and 5, and, e.g., Wang \& Heckman 1996; Calzetti et al. 2007). Thus, the $70 \mu \mathrm{m}$ emission becomes "underluminous" because the dust emission does not fully trace the light from star formation. Furthermore, the peak of the infrared emission shifts to longer wavelengths for decreasing IRSD values (Figure 5 and Draine et al. 2007), exacerbating the trend. If we constrain the fitting range to increasingly large SFSDs, the slope in Equation (18) tends asymptotically to unity; it is about $2 \sigma$ away from unity for $\log \left(\Sigma_{\mathrm{SFR}}\right)>-1$, and less than $1 \sigma$ away from unity for $\log \left(\Sigma_{\mathrm{SFR}}\right)>-0.6$.

An independent test is to use the luminosities or SFRs normalized by the $3.6 \mu \mathrm{m}$ luminosity, $L(3.6)$ (Figure 10), since this proxy for stellar mass is not dominated by the current star formation. The best-fit line through the data is

$$
\begin{aligned}
\log [L(70) / L(3.6)]= & (1.05 \pm 0.02) \log [\mathrm{SFR} / L(3.6)] \\
& +(45.30 \pm 0.02),
\end{aligned}
$$

where SFR is from Equation (17), and the units of SFR/L(3.6) are $M_{\odot} \mathrm{yr}^{-1}\left(\mathrm{erg} \mathrm{s}^{-1}\right)^{-1}$. The slope in Equation (19) is also slightly above unity, only $2.5 \sigma$ away from it, and quickly converges to unity for a fitting range that excludes the faintest galaxies, in agreement with our conclusions above.

The simple stellar population and dust emission models briefly presented in Section 4.1 can further help clarify some of the observed trends. At the high-luminosity end, the models have slope roughly unity by construction (Figure 5), because in this regime TIR is a good proxy for SFR; thus the trend $\Sigma_{70}-\Sigma_{\text {SFR }}$ does not provide independent information. For SFSD $\gtrsim 0.5 M_{\odot} \mathrm{yr}^{-1} \mathrm{kpc}^{-2}$, the opacity of the system is high and most of the stellar light is reprocessed by dust into the infrared. Thus, the details of the extinction model are unimportant, and the normalization factor between the SFSD and the total infrared emission is provided by the stellar population. The mean trend of the $70 \mu \mathrm{m}$ surface brightness is well represented by a model where a $100 \mathrm{Myr}$ constant star formation population heats the dust (Figure 11), which is how we describe LIRGs (Equation (17)).

As the SFSD decreases, the average opacity of the galaxy also tends to decrease (Wang \& Heckman 1996; Heckman et al. 1998; Hopkins et al. 2001; Calzetti 2001). The opacity models of Calzetti et al. (2007, constructed for $\mathrm{H}$ II regions), however, underpredict the observed $\Sigma_{70}$ by a factor $\approx 2-3$, for $\Sigma_{\mathrm{SFR}} \lesssim$ $0.01 M_{\odot} \mathrm{yr}^{-1} \mathrm{kpc}^{-2}$ (Figure 11). All the other models, the extreme case of constant and large dust opacity for the entire SFSD range, the model with a filling factor of the emitting regions of about $10 \%$ of the normalizing area, and the stellar population models with exponentially decreasing star formation, mark a range that brackets the spread of the data points. The mean trend of the data at low SFSDs is better represented by the model with a $10 \%$ filling factor of the emitting regions, for constant star formation. This is perhaps not surprising, since star formation in low surface brightness galaxies tends to display a morphology of isolated knots distributed over a large, noncontiguous area, while our definition of "ionized gas emitting area" (Section 2) measures a single size per galaxy, irrespective 

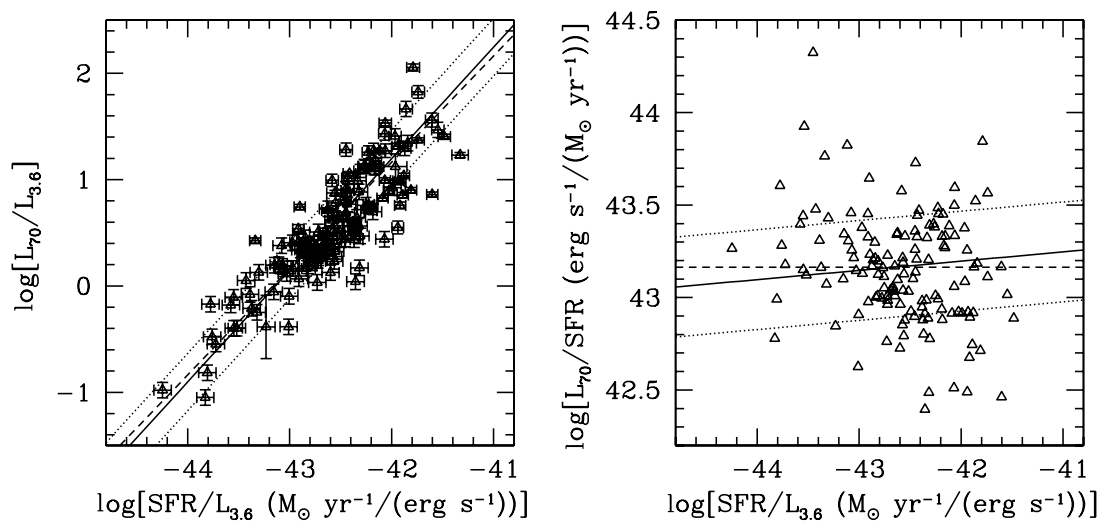

Figure 10. Left and right: the same as Figure 9, with the data normalized by the $3.6 \mu \mathrm{m}$ luminosity, instead of the emitting area. The same data are shown, together with the best fit (continuous line) and the $1 \sigma$ envelope to the data (dotted lines, which correspond to the range $-0.27,+0.27$ dex). The best fit with unity slope is shown as a dashed line in both panels.
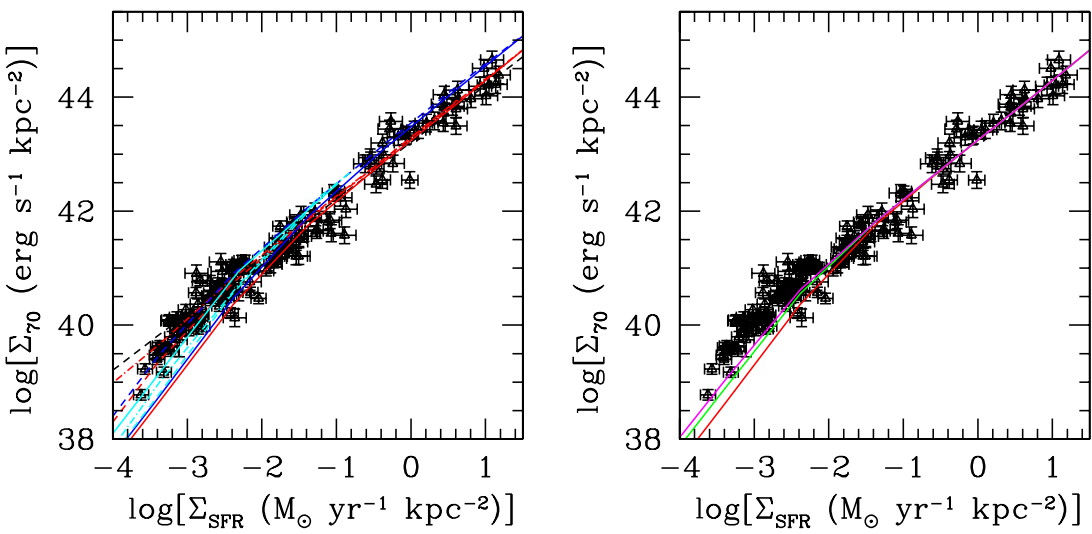

Figure 11. Same data on the $70 \mu \mathrm{m} \mathrm{LSD}, \Sigma_{70}$, vs. the SFSD, $\Sigma_{\mathrm{SFR}}$ as in Figure 9. Left: the data are compared with the simple galaxy models described in Figure 4 (see the text for more details). The best linear fit with unity slope is shown as a black dashed line (from Figure 9). All stellar population models are for solar metallicity: $10 \mathrm{Gyr}$ old (blue continuous) and $100 \mathrm{Myr}$ old (red continuous) constant star formation populations, and exponentially decreasing star formation (cyan; dash: $\tau=$ 5 Gyr; continuous: $\tau=2 \mathrm{Gyr}$ ), together with our default dust model, in which the dust extinction is described by the starburst curve (Calzetti 2001), the dust emission comes from a medium of increasing opacity, and the peak infrared emission progressively moves into the $70 \mu \mathrm{m}$ band from longer wavelengths (Draine \& Li 2007) for increasing SFSD. Dashed red and blue lines show the same default dust models, but the star-forming regions have a $10 \%$ filling factor within the galaxies, for stellar population with $100 \mathrm{Myr}$ and $10 \mathrm{Gyr}$ constant star formation, respectively. The case of a constant and large dust opacity ( $A_{V}>10 \mathrm{mag}$ ) with SFSD is shown as a red dot-dashed line for a $100 \mathrm{Myr}$ constant star formation population. The cyan dot-dashed line is a decreasing star formation model, with $\tau=2 \mathrm{Gyr}$, embedded in a uniform distribution of dust (mixed dust-star case). Right: the $100 \mathrm{Myr}$ constant star formation model is here reproduced for three different dust attenuation curves: the starburst curve (red line), used in our default model, according to which stars and ionized gas display differential attenuation (by a factor 0.44, see Calzetti et al. 1994; Calzetti et al. 2000); the Milky Way (green line) and SMC (magenta line) extinction curves, with foreground dust geometry, and stars and gas attenuated by the same dust column density.

of the distribution and filling factor of the emitting regions. In the case of exponentially decreasing star formation, the mean trend of the data requires higher filling factors, around $30 \%-50 \%$ for the model with $e$-folding time $\tau=2 \mathrm{Gyr}$, but still around $15 \%$ for the $5 \mathrm{Gyr} e$-folding time model.

Our solutions are not unique, and different combinations of dust opacity models and filling factors can provide similar trends. For instance, the extreme case of a foreground dust geometry for the galaxy, with a Small Magellanic Cloud (SMC) extinction curve (Bouchet et al. 1985) and common dust column density to both stars and ionized gas gives higher values of the infrared emission at low luminosities than the starburst attenuation curve (Figure 11, right). Thus, the low-luminosity data points can be accounted for by a larger filling factor of the emitting regions, $\sim 30 \%$, about a factor 3 larger than the case of the starburst attenuation curve with constant star formation. The conclusion is still that a low filling factor is needed for the emitting regions, between $10 \%$ and $30 \%$, for constant star formation, and around $15 \%-50 \%$ for exponentially decreasing star formation, in order to account for the observed trend between the $70 \mu \mathrm{m}$ emission and the SFSD for $\Sigma_{\mathrm{SFR}} \lesssim$ $0.01 M_{\odot} \mathrm{yr}^{-1} \mathrm{kpc}^{-2}$.

The overall trend of the high-metallicity data points is consistent with both increasing dust opacity and a smooth transition from a low filling factor to a high filling factor as the SFSD increases, with the transition point located around $\Sigma_{\mathrm{SFR}} \sim 0.01 M_{\odot} \mathrm{yr}^{-1} \mathrm{kpc}^{-2}$.

Including the low-metallicity data in the $\Sigma_{70}-\Sigma_{\mathrm{SFR}}$ plot provides further insights into the effect of dust opacity, as lowmetallicity galaxies tend to be more transparent than highermetallicity ones (Cannon et al. 2005; Walter et al. 2007). These data are located typically below the trend marked by the high-metallicity data (Figure 12), as expected for decreasing dust amounts in the ISM. The inclusion of the model line for a $1 / 10$ solar metallicity ISM from Calzetti et al. (2007) shows that metallicity has a major effect on the location of galaxies on the plot. The model line for low-metallicity systems is located between 4 times (at high luminosity) and an order of magnitude (at low luminosity) below the analogous model for solar metallicity systems. There are a few galaxies 


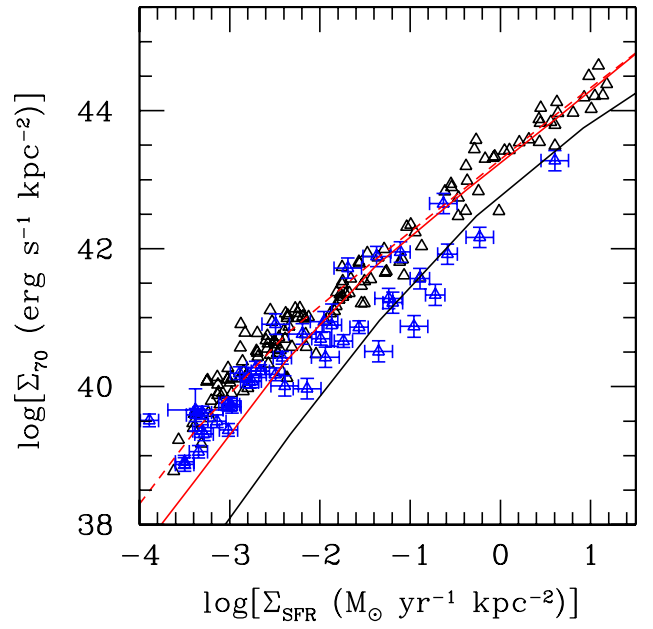

Figure 12. Data on the $70 \mu \mathrm{m} \mathrm{LSD}, \Sigma_{70}$, vs. the SFSD, $\Sigma_{\mathrm{SFR}}$ now include both metal-rich (black symbols) and metal-poor (blue symbols) galaxies, as defined in the text. $1 \sigma$ error bars are reported for the low-metallicity data points, while the error bars for the metal-rich galaxies are omitted for clarity. The model of a $100 \mathrm{Myr}$ continuous star formation population is used for reference, from Figure 11. The default solar metallicity model (red continuous line) and the $10 \%$ filling-factor one (red dashed line) are compared with a 0.1 solar metallicity model (continuous black line, from Calzetti et al. 2007).

(A color version of this figure is available in the online journal.)

that are located even below our low-metallicity model line (Figure 12). Two galaxies, Tol65 and SBS0335-052, are more than $3 \sigma$ below the low-metallicity curve, while IZw 18 is about $2 \sigma$ below the curve. These three galaxies, with metallicities between 7.20 and 7.45 (Nagao et al. 2006), are among the most metal poor in our sample, and well below 1/10th solar. Furthermore, Tol65 and SBS0335-052 have high effective dust temperature and their peak infrared emission is located shortward of $70 \mu \mathrm{m}$ (Engelbracht et al. 2008). Thus, it is not surprising that the three galaxies lie below our $1 / 10$ th solar model line.

Although metallicity has an important effect on the applicability of the $70 \mu \mathrm{m}$ emission as an SFR indicator, LIRGs tend to be, on average, metal and dust rich. Indeed, most of the lowmetallicity galaxies in our sample cluster at the low-luminosity and low $\Sigma_{\text {SFR }}$ end of the range (Figure 12), an effect of typically "hotter" IR SEDs relative to the high-metallicity galaxies (Dale et al. 2005). From the discussion above, we approximate the trend of the high-metallicity data (Figure 13) with

$$
\Sigma_{\mathrm{SFR}}\left(M_{\odot} \mathrm{yr}^{-1} \mathrm{kpc}^{-2}\right)=\frac{\Sigma_{70}\left(\mathrm{erg} \mathrm{s}^{-1} \mathrm{kpc}^{-2}\right)}{1.7 \times 10^{43}},
$$

for $7 \times 10^{40} \mathrm{erg} \mathrm{s}^{-1} \mathrm{kpc}^{-2} \lesssim \Sigma_{70} \lesssim 5 \times 10^{44} \mathrm{erg} \mathrm{s}^{-1} \mathrm{kpc}^{-2}$, and

$$
\Sigma_{\mathrm{SFR}}\left(M_{\odot} \mathrm{yr}^{-1} \mathrm{kpc}^{-2}\right)=\left(\frac{\Sigma_{70}\left(\mathrm{erg} \mathrm{s}^{-1} \mathrm{kpc}^{-2}\right)}{3.3 \times 10^{44}}\right)^{0.65},
$$

for $6 \times 10^{38} \mathrm{erg} \mathrm{s}^{-1} \mathrm{kpc}^{-2} \lesssim \Sigma_{70} \lesssim 7 \times 10^{40} \mathrm{erg} \mathrm{s}^{-1} \mathrm{kpc}^{-2}$. The nonlinear relation between the $70 \mu \mathrm{m} \mathrm{LSD}$ and the SFSD (Equation (21)) attempts to take into account the increasing transparency of the medium for decreasing SFSDs, and is a rough approximation to a more complicated trend (Figure 13). The important caveat about Equation (21) is that the calibration is highly dependent on the type of objects from which it is derived (whole galaxies in this case), and should not be applied to different physical systems (e.g., H II regions or complexes

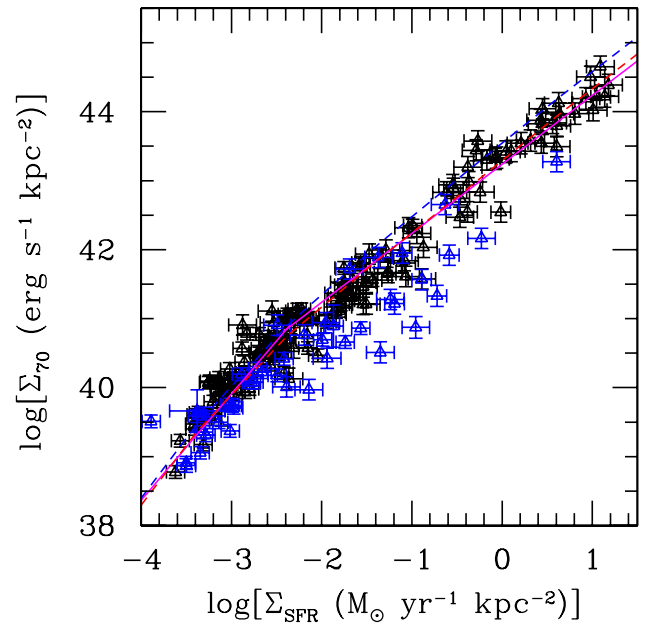

Figure 13. Data on the $70 \mu \mathrm{m} \mathrm{LSD}, \Sigma_{70}$, vs. the SFSD, $\Sigma_{\mathrm{SFR}}$ for both metal-rich (black symbols) and metal-poor (blue symbols) galaxies. The data are the same as in Figure 12, except that now error bars are included for all data points. Models of stellar populations with constant star formation over $100 \mathrm{Myr}$ (red dashed line) and $10 \mathrm{Gyr}$ (blue dashed line) surrounded by a solar metallicity ISM with a $10 \%$ area filling factor at the low-luminosity end are also reported, and compared with the best power-law fits to the asymptotic trends at the lowand high-luminosity ends (broken magenta line, Equations (20) and (21)).

(A color version of this figure is available in the online journal.)

within galaxies) or metal-poor systems, without proper checks. Nevertheless, the two power laws, Equations (20) and (21), provide a reasonable description of the data points in our sample with $12+\log (\mathrm{O} / \mathrm{H}) \gtrsim 8.1$, which show a relatively symmetric dispersion around those mean trends, with $1 \sigma \sim 0.2$ dex (Figure 14), or about $60 \%$ in linear scale. The linear relation at the high-luminosity end offers the opportunity to derive directly an SFR:

$$
\operatorname{SFR}(70)\left(M_{\odot} \mathrm{yr}^{-1}\right)=\frac{L(70)\left(\mathrm{erg} \mathrm{s}^{-1}\right)}{1.7 \times 10^{43}},
$$

applicable to luminosities $L(70) \gtrsim 1.4 \times 10^{42} \mathrm{erg} \mathrm{s}^{-1} \sim 3.7 \times$ $10^{8} L_{\odot}$. Again, the relation between luminosity and luminosity/ area has at least 1 order of magnitude dispersion in our sample. Adopting the SFR $-L(\mathrm{H} \alpha)$ calibration of Kennicutt (1998) would change the denominator in Equation (22) to $1.2 \times 10^{43}$. The change is due to a combination of adopted stellar IMF (Kennicutt 1998, adopts a Salpeter IMF in the range 0.1-100 $M_{\odot}$ ) and small $(\sim 10 \%)$ variations in the stellar population models.

\section{CAN WE ALSO DERIVE SFR(160)?}

The $160 \mu \mathrm{m}$ LSD correlates, like $\Sigma_{70}$, with SFSD, with linear best fit (Figure 15):

$$
\begin{aligned}
& \log \left[\Sigma_{160}\left(\mathrm{erg} \mathrm{s}^{-1} \mathrm{kpc}^{-2}\right)\right]=(0.954 \pm 0.012) \\
& \quad \times \log \left[\Sigma_{\mathrm{SFR}}\left(M_{\odot} \mathrm{yr}^{-1} \mathrm{kpc}^{-2}\right)\right]+(42.910 \pm 0.028) .
\end{aligned}
$$

The shallower-than-unity slope is consistent with our interpretation that galaxies of decreasing luminosity have "cooler" infrared SEDs (Figure 5). This result is partially counteracted by a sharp decline in $160 \mu \mathrm{m}$ luminosity for $\log \left(\Sigma_{\mathrm{SFR}}\right)<-3.2$, indicating the regime where the galaxies become measurably transparent. Like in the case of the $70 \mu \mathrm{m} \mathrm{LSD}$, the best-fit line tends asymptotically to a slope of unity for galaxies of increasing SFSD; in the case of the $160 \mu \mathrm{m} \mathrm{LSD}$, the best fit has slope of unity within $1 \sigma$ already for $\log \left(\Sigma_{\mathrm{SFR}}\right)>-2$. 


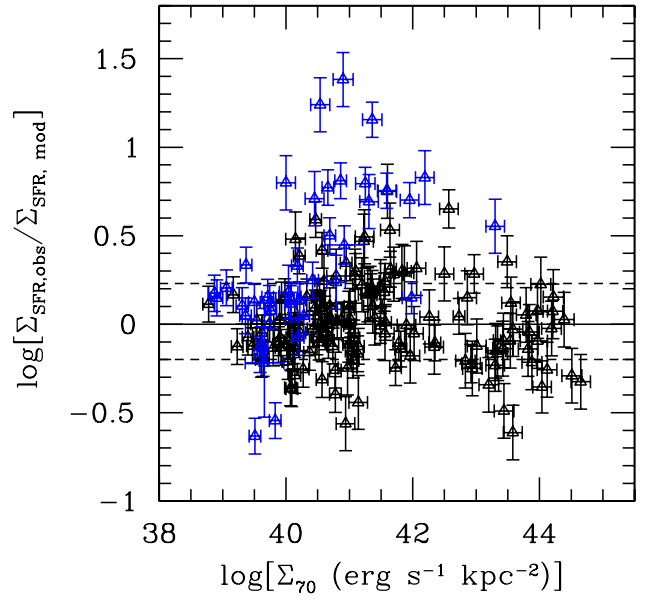

Figure 14. Difference between the data and the fitting lines described in Equations (20) and (21) is reported as a function of the $70 \mu \mathrm{m} \mathrm{LSD}$, together with horizontal lines showing identity between models and data (continuous line) and the $1 \sigma$ dispersion value $(-0.20,+0.23$, dashed lines).

The dispersion of the data about the best-fit line, $(-0.42$, $+0.34)$ dex, is larger for $\Sigma_{160}$ than for $\Sigma_{70}$ by a factor $\sim 1.3-1.5$ (Figures 9 and 14, left). A similar ratio, $\sim 1.2-1.4$, between the $1 \sigma$ dispersions holds when quantities normalized to the $3.6 \mu \mathrm{m}$ luminosity are considered (Figure 16). Furthermore, the dispersion on the positive side, i.e., for large $160 \mu \mathrm{m}$ emission relative to the SFSD, is dominated by galaxies at the low SFSD end, $\log \left(\Sigma_{\mathrm{SFR}}\right)<-2$ (Figure 15, right). These two facts, when taken together, suggest that the $160 \mu \mathrm{m}$ luminosity is affected by emission from dust heated by stellar populations unrelated to those powering the current star formation. This effect becomes more and more evident as total luminosities decrease. Indeed, the same models with $10 \%$ area filling factor of the emitting regions that satisfactorily account for the trend of the data in the $\Sigma_{70}-\Sigma_{\text {SFR }}$ plane provide an unsatisfactory description of the data in the $\Sigma_{160}-\Sigma_{\text {SFR }}$ plane for $\Sigma_{\text {SFR }}<0.01$, for both the $100 \mathrm{Myr}$ and the $10 \mathrm{Gyr}$ constant star formation population: the models underpredict, by roughly 0.5 dex or more, the median locus of the data for most of the data in this SFSD range (Figure 15, left). A similar result is obtained when normalizing the $160 \mu \mathrm{m}$ luminosity and the SFR to the $3.6 \mu \mathrm{m}$ luminosity, instead of the area (Figure 16). In this case, the best fit to the data has a shallower-than-unity slope:

$$
\begin{aligned}
\log [L(160) / L(3.6)]= & (0.87 \pm 0.01) \log [\mathrm{SFR} / L(3.6)] \\
& +(37.48 \pm 0.02)
\end{aligned}
$$

(SFR is from Equation (17), and the units of $\mathrm{SFR} / L(3.6)$ are $\left.M_{\odot} \mathrm{yr}^{-1}\left(\mathrm{erg} \mathrm{s}^{-1}\right)^{-1}\right)$ and the slope approaches unity only for increasingly luminous galaxies. Equation (24) supports, again, the presence of two effects for low-luminosity galaxies: "cooler" infrared SEDs for lower luminosity galaxies and a proportionally increasing contribution, for decreasing luminosity, to the $160 \mu \mathrm{m}$ emission from dust heated by stellar populations that are unassociated with the current star formation.

All of the above strengthens the consideration made in Section 5 that a fraction of the dust emission detected in the $70 \mu \mathrm{m}$ band toward low SFSDs is due to stellar populations unrelated to the current star formation, and this effect is, expectedly, larger in the $160 \mu \mathrm{m}$ showing up as both a larger dispersion of the data around the mean trends and an excess deviation of the data from the model predictions at the lowluminosity end.

This conclusion is not new: many authors have noted over the past $\sim 2$ decades the presence of a contribution to the infrared emission of galaxies from stellar populations not associated to the current star formation (e.g., Lonsdale Persson \& Helou 1987; Sauvage \& Thuan 1992; Buat \& Xu 1996; Walterbos \& Greenawalt 1996). The presence of such contribution limits the usefulness, especially at low galaxy luminosity, of SFR calibrations based on monochromatic IR emission longward of the SED peak.

In summary, although defining an SFR calibration based on the $160 \mu \mathrm{m}$ emission may be tempting:

$$
\operatorname{SFR}(160)\left(M_{\odot} \mathrm{yr}^{-1}\right)=\frac{L(160)\left(\mathrm{erg} \mathrm{s}^{-1}\right)}{7.0 \times 10^{42}},
$$

with applicability for $L(160)>1 \times 10^{42} \mathrm{erg} \mathrm{s}^{-1} \sim 2.6 \times 10^{8} L_{\odot}$, the presence of a large dispersion, $1 \sigma_{160} \sim 2 \times\left(1 \sigma_{70}\right)$, and the likelihood that a portion of the $160 \mu \mathrm{m}$ emission (and a large fraction of it at the low-luminosity end) is due to dust heating by non-star-forming populations prevents such definition from having the same confidence level as the calibration of SFR(70).
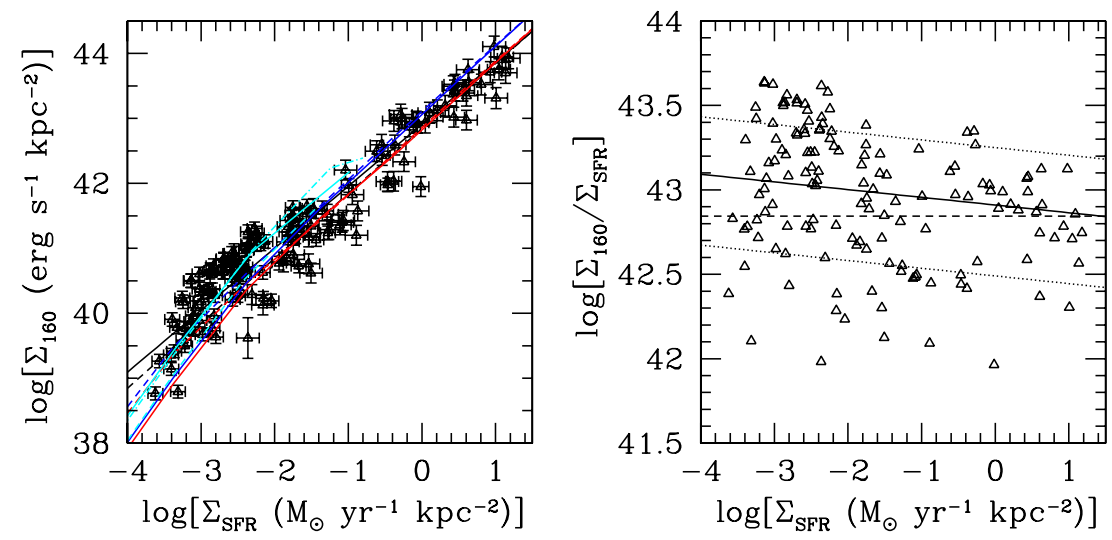

Figure 15. Left: the $160 \mu \mathrm{m} \mathrm{LSD}, \Sigma_{160}=L(160) /$ area, vs. the SFSD, $\Sigma_{\mathrm{SFR}}$, for the metal-rich galaxies in our sample. Both best linear fits and model lines are overplotted on the data. The best fit has a slope slightly less than unity (continuous black line), and a line through the data with unity slope is shown as a dashed black line. The models are the same as Figure 11. Right: similarly to the right-hand panel of Figure 9, the same data and best linear fit (continuous line) are shown, together with the $1 \sigma$ envelope to the data (dotted lines), for the ratio $L(160) /$ SFR as a function of the SFSD. The best fit with unity slope is shown as a dashed line. This plot better shows the deviation of the data points from a one-to-one relation. The error bars on the data are not shown, for clarity. 

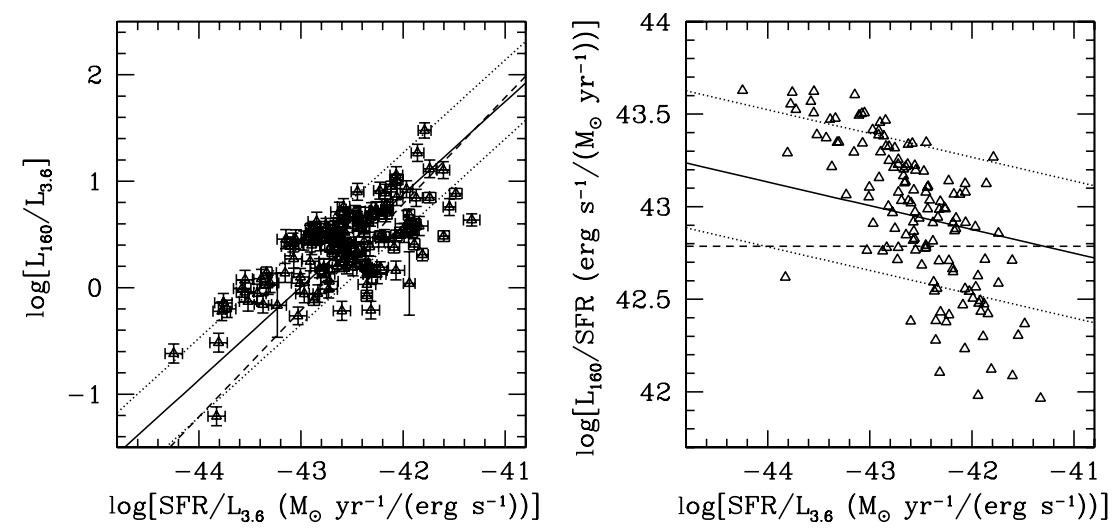

Figure 16. Left and right: the same data as Figure 15, with the data normalized by the $3.6 \mu \mathrm{m}$ luminosity, instead of the emitting area. The best fit (continuous line) and the $1 \sigma$ envelope to the data (dotted lines, which correspond to the range $-0.35,+0.39$ dex) are also shown. A line through the data with unity slope is shown in both panels (dashed line).

As will be seen in the next section, a safer range of applicability of Equation (25) is for $L(160)>2 \times 10^{42} \mathrm{erg} \mathrm{s}^{-1} \sim 5.2 \times$ $10^{8} L_{\odot}$. With the Kennicutt (1998) calibration for SFR, the numerical constant in Equation (25) changes to $4.8 \times 10^{42}$.

\section{DISCUSSION AND CONCLUSIONS}

Monochromatic SFR indicators have the undeniable advantage of immediate application, especially when observational data cover only a limited range of wavelengths, as is often the case for distant galaxies. Within this framework, Spitzer observations of nearby galaxies are offering a unique opportunity to test the applicability and limitations of a number of SFR indicators based on the emission from the dust heated by stars. The main goal of this paper has been to investigate whether longwavelength infrared fluxes at 70 and $160 \mu \mathrm{m}$ can be reasonably calibrated as SFR indicators.

Previous works (Roussel et al. 2001; Boselli et al. 2004; Förster Schreiber et al. 2004; Calzetti et al. 2005, 2007; Dale et al. 2005; Wu et al. 2005; Alonso-Herrero et al. 2006; PerezGonzalez et al. 2006; Dale et al. 2007; Relaño et al. 2007; Zhu et al. 2008; Rieke et al. 2009) have concentrated on the short-wavelength infrared, in the range $\sim 5-40 \mu \mathrm{m}$, commonly referred to as the mid-IR. Although this wavelength range is below the peak infrared emission and only accounts for a few to a few tens of percent of the total IR emission, the dust heated by the hot, massive stars in a recent star formation episode can have high effective temperatures and may preferentially emit at the shorter infrared wavelengths. This general picture translates into a correlation between SFRs and luminosities in the mid-IR, such as $L(8)$ and $L(24)$, centered on the Spitzer $8 \mu \mathrm{m}$ and $24 \mu \mathrm{m}$ bands. However, the correlation is also nonlinear, and careful analysis shows that other contributors, in addition to the SFR, determine the short infrared wavelength luminosity, including transiently heated dust by single UV or optical photons in the general radiation field of a galaxy (Haas et al. 2002; Boselli et al. 2004; Bendo et al. 2008), variations in the dust effective temperature (for $L(24)$, see Calzetti et al. 2007), and, especially for $L(8)$, gas metallicity (Engelbracht et al. 2005; Rosenberg et al. 2006; Wu et al. 2006; Madden et al. 2006; Jackson et al. 2006; Draine et al. 2007).

\subsection{Establishing a Reference SFR Indicator}

In the present paper, we first establish a "reference" SFR calibration against which we can compare the 70 and $160 \mu \mathrm{m}$ luminosities and LSDs. The adopted reference SFR is given in
Equation (17). We choose this particular three-part formulation after comparing a variety of published $24 \mu \mathrm{m}$ and composite $\mathrm{H} \alpha$ plus $24 \mu \mathrm{m}$ calibrations.

Above a luminosity $L(24) \sim 5 \times 10^{43} \mathrm{erg} \mathrm{s}^{-1}$, the contribution of direct (unabsorbed by dust) massive stars emission to SFR measurements is less than $\sim 15 \%$ (Figure 8). For $L(24) \gtrsim 5 \times$ $10^{43} \mathrm{erg} \mathrm{s}^{-1}$, the $24 \mu \mathrm{m}$ emission is a reliable SFR indicator for whole galaxies. However, in this luminosity regime, the $24 \mu \mathrm{m}$ dust emission is also self-absorbed (Rieke et al. 2009), and a nonlinear correction to the calibration needs to be applied (third term of Equation (17), re-calibrated from the original calibration of Rieke et al. 2009).

Below a luminosity $L(24) \sim 5 \times 10^{43} \mathrm{erg} \mathrm{s}^{-1}$, the galaxies become transparent in the mid-infrared, and, as the luminosity further decreases, they become transparent at UV and optical wavelengths (e.g., Bell 2003; Buat et al. 2007). At L(24) $4 \times 10^{42} \mathrm{erg} \mathrm{s}^{-1}$, the contribution to the SFR from emission at optical $(\mathrm{H} \alpha)$ and IR $(24 \mu \mathrm{m})$ wavelengths is roughly equal (Figure 7). Thus, below $L(24) \sim 5 \times 10^{43} \mathrm{erg} \mathrm{s}^{-1}$, the $24 \mu \mathrm{m}$ luminosity becomes increasingly insufficient, by itself, to fully characterize the SFR of a whole galaxy, as the portion of the star formation unabsorbed by dust becomes an increasingly significant contribution to the total SFR. Additionally, a correlation between the effective temperature and the luminosity/area of the thermal dust emission is present in $\mathrm{H}$ II regions for decreasing total SFRs (Calzetti et al. 2007), and in whole galaxies as well (Chanial et al. 2007 and Figure 4). This correlation is further seen for the galaxies in our sample by plotting the ratio $L(70)$ / $L(24)$ as a function of $\Sigma_{24}$ (Figure 17, left); the star-forming and starburst galaxies show a decreasing IR ratio for increasing LSD, as is expected for increasing effective dust temperature. This trend is less tight, albeit still present to some extent, when the $L(70) / L(24)$ ratio is plotted as a function of luminosity (Figure 17, right), rather than luminosity/area. The use of total luminosity introduces a scatter because galaxies are weighted not only by their intrinsic level of activity but also by their size (a low-activity, low IR/area, but large, galaxy can have the same total luminosity as a high activity, but small, galaxy).

The relation between the effective temperature and the luminosity/area is present in galaxies up to an IRSD $\Sigma_{\text {TIR }} \sim$ $0.3-1 \times 10^{43} \mathrm{erg} \mathrm{s}^{-1} \mathrm{kpc}^{-2}\left(L(\mathrm{TIR}) \approx 1-3 \times 10^{44} \mathrm{erg} \mathrm{s}^{-1}\right.$ $\sim 3-8 \times 10^{10} L_{\odot}$ or $L(24) \approx 2-6 \times 10^{43} \mathrm{erg} \mathrm{s}^{-1} \sim 0.6-2$ $\times 10^{10} L_{\odot}$ ), independent of the metal content of the galaxy (Figures 3, left, and 17). This can be further seen in Figure 18 , where we plot $L(24) / L(T I R)$ as a function of IRSD only for those galaxies in our sample that have oxygen abundance 

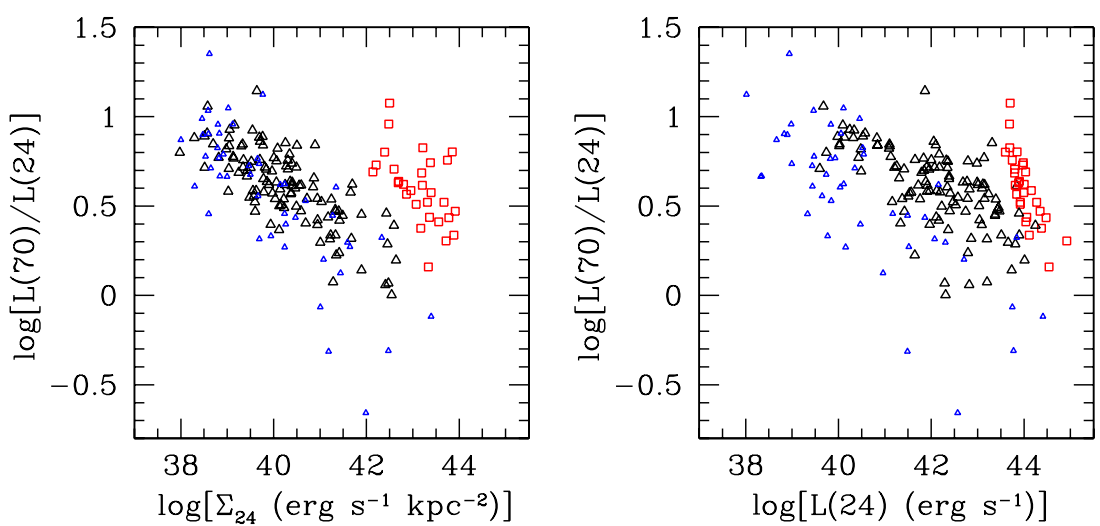

Figure 17. $L(70) / L(24)$ ratio as a function of the $24 \mu \mathrm{m} \mathrm{LSD}, \Sigma_{24}$, (left) and luminosity, $L(24)$, (right), respectively. As in Figure 1, colors identify the star-forming and starburst high-metallicity (black symbols) and low-metallicity (blue symbols) galaxies, and the LIRGs (red). Error bars are omitted for clarity. The decreasing trend of $L(70) / L(24)$ as a function of increasing LSD for normal star-forming and starburst galaxies (black and blue symbols) observed in the left-hand-side panel shows the variation in the dust effective temperature for these systems, similarly to Figure 3 . The trend shows a large scatter, when the ratio $L(70) / L(24)$ is plotted as a function of luminosity (right-hand side panel).

$12+\log (\mathrm{O} / \mathrm{H})>8.5$. There are 66 normal star-forming and starburst galaxies above this oxygen abundance value (or about $1 / 2$ of the normal star-forming and starburst galaxies in our high-metallicity sample), and the 29 LIRGS. A similar trend to that of Figure 4 of $L(24) / L(T I R)$ as a function of IRSD is observed for this restricted, higher-metallicity, sample, implying that the effect is not primarily driven by the abundance of dust in the galaxy.

The combination of the two effects discussed above, increasing transparency of the galaxy and decreasing effective dust temperature for decreasing luminosity/area, may account for why different published calibrations for SFR(24) diverge when extrapolated towards the low-luminosity end of our sample (Figure 6).

A more robust approach appears to involve measuring SFRs by combining the $24 \mu \mathrm{m}$ emission and the observed $\mathrm{H} \alpha$ emission, the first probing the dust-absorbed star formation and the second the unabsorbed one (Kennicutt et al. 2007, 2009; Calzetti et al. 2007). Even in this case, though, a transition in the proportionality factor between $L(24)$ and $L(\mathrm{H} \alpha)_{\text {obs }}$ needs to be implemented for estimating accurate SFRs (Equation (17), first and second terms). The transition point is marked by the condition $L(\mathrm{H} \alpha)_{\text {obs }} /(0.020 L(24)) \approx 1$, which corresponds, for decreasing luminosity, to the transition from galaxies dominated by dustobscured star formation to those dominated by unobscured star formation (e.g., Buat et al. 2007). The transition corresponds to a change of roughly $50 \%$ in the fraction of $L(24)$ to be added to $L(\mathrm{H} \alpha)_{\text {obs }}$, from 0.031 to 0.020 for $L(24) \lesssim 4 \times 10^{42} \mathrm{erg} \mathrm{s}^{-1}$ (first and second relation in Equation (17)), or SFR $\sim 1 M_{\odot} \mathrm{yr}^{-1}$. Our results thus indicate that around this SFR, the stellar population that dominates the heating of the dust transitions from being a relatively young one (constant star formation over the past $\sim 100 \mathrm{Myr}$ ), typical of starburst events, to a much more evolved one (constant star formation over the past $\sim 10 \mathrm{Gyr}$, or exponentially decreasing star formation), typical of widespread, but isolated, star formation amid an otherwise quiescent galaxy. In the latter case, the stellar populations not associated with the current star formation events contribute, in a proportionally larger fraction than the starburst case, to the heating of the $24 \mu \mathrm{m}$ emitting dust, whose emission contribution to the SFR calibration needs then to be accordingly removed, on average, by reducing $L(24)$ via a smaller multiplicative factor (Kennicutt et al. 2009) than the starburst or H II region case (Calzetti et al. 2007).

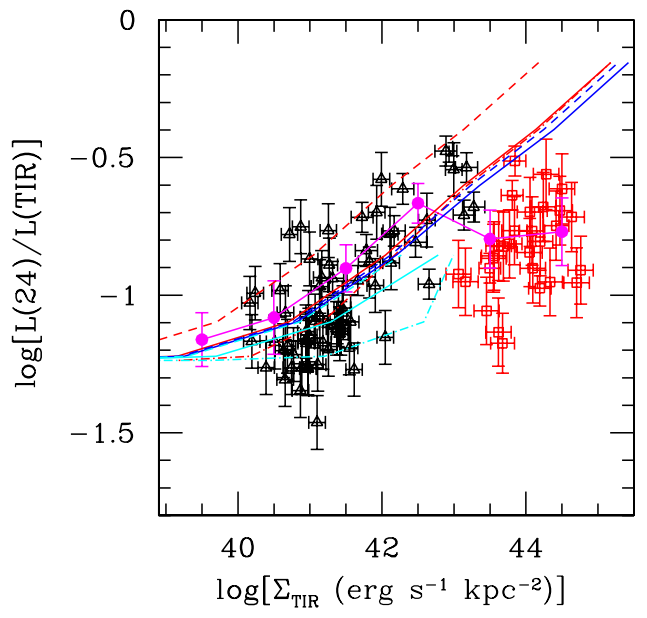

Figure 18. $L(24) / L($ TIR) ratio as a function of the IRSD for the galaxies in our sample with oxygen abundance $12+\log (\mathrm{O} / \mathrm{H})>8.5$ (black and red points). The magenta points and line, and the models lines (blue, red, and cyan) are as in Figure 4, left. The trend for the galaxies with $12+\log (\mathrm{O} / \mathrm{H})>8.5$ is similar to that of the whole sample with $12+\log (\mathrm{O} / \mathrm{H})>8.1$.

\subsection{The Emission at 70 and $160 \mu m$ as SFR Indicators}

Our reference SFR calibration (Equation (17)) enables the testing of other IR monochromatic luminosities as potential SFR indicators, over a wide range of LSDs. In this work, we have investigated the applicability of $L(70)$ and $L(160)$, i.e., the long-wavelength Spitzer bands, as such indicators. These two wavelength regions are attractive because each contains a larger fraction of the total infrared emission than the $24 \mu \mathrm{m}$ emission. $L(70)$ is $50 \%$ of $L\left(\right.$ TIR) for infrared LSD $\Sigma_{\text {TIR }} \gtrsim$ $10^{41} \mathrm{erg} \mathrm{s}^{-1} \mathrm{kpc}^{-2}$, and $L(160)$ is about $20 \%$ or larger fraction of $L(T I R)$ for the entire luminosity range investigated in this work (Figure 5), while $L(24)$ only contains $\sim 10 \%-20 \%$ of the total infrared emission (Figure 4). At the high-luminosity LIRGs regime, both $L(70)$ and $L(160)$ represent a larger fraction of $L$ (TIR) than expected from models (e.g., Draine et al. 2007). This indicates a "cooler" (larger effective temperature) IR SED for those systems than predicted from models, an effect already previously observed (Dunne \& Eales 2001), and in line with the re-emission of the energy absorbed in the mid-IR wavelength range. Galaxies in the LIRG luminosity regime ( $L(T I R)$ $\gtrsim 1-3 \times 10^{44} \mathrm{erg} \mathrm{s}^{-1}$ or $\left.\Sigma_{\mathrm{TIR}} \gtrsim 0.3-1 \times 10^{43} \mathrm{erg} \mathrm{s}^{-1} \mathrm{kpc}^{-2}\right)$ 

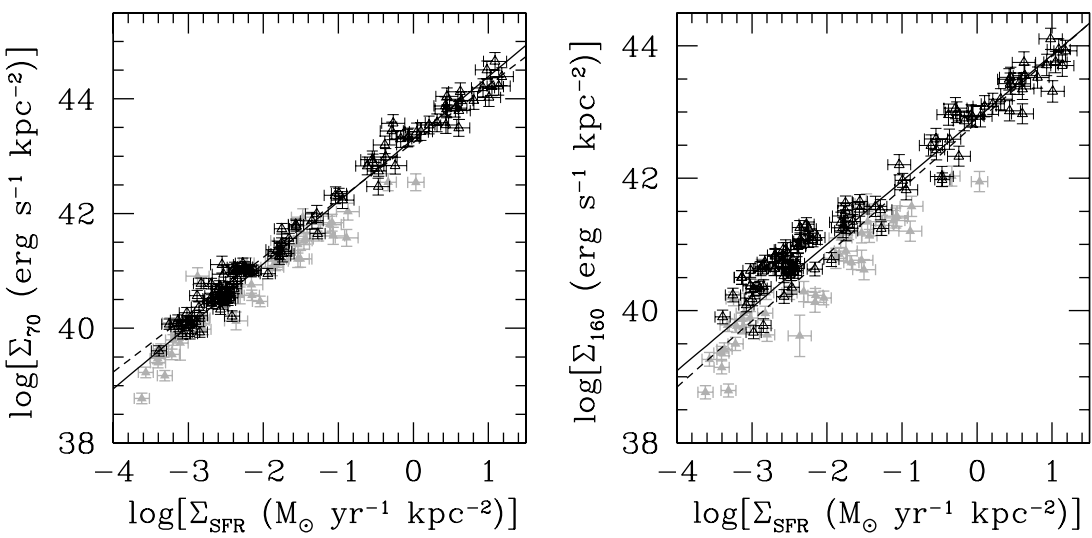

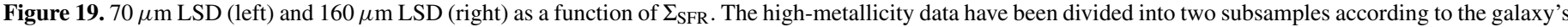
oxygen abundance: $8.1<12+\log (\mathrm{O} / \mathrm{H}) \leqslant 8.5$ (gray triangles) and $12+\log (\mathrm{O} / \mathrm{H})>8.5$ (black triangles). See the text for an explanation of the selected metallicity boundary. The scatter in the data decreases when restricting the sample to the highest metallicity values. The continuous and dashed lines in both panels are the best fit (Equations (18) and (23)) and the unity slope lines, respectively.

have their energy output dominated by a star formation event that is evolving in a dusty region of sufficient optical depth that even light emerging in the mid-IR gets absorbed, and that light is re-emitted at longer infrared wavelengths, thus producing an overall cool IR SED, specifically cooler than expected from models where the emission is emerging from a transparent (at IR wavelengths) medium. This requires that the dust attenuation at $24 \mu \mathrm{m}$ be substantial, $\approx 1 \mathrm{mag}$, which translates to $A_{V} \sim 15-50 \mathrm{mag}$, depending on the extinction curve adopted (Draine \& Li 2007; Flaherty et al. 2007). Such large extinctions are not uncommon in bright infrared galaxies. Genzel et al. (1998) determine that ultraluminous infrared galaxies (ULIRGs) have extinction values in the range $A_{V \text {,screen }}=5-50 \mathrm{mag}$ or $A_{V \text {,mixed }}=50-1000 \mathrm{mag}$. Although LIRGs are typically an order of magnitude less luminous than ULIRGs, dust extinctions are still expected to be large in these systems.

The $70 \mu \mathrm{m}$ emission from galaxies correlates linearly with the SFR for luminosities $L(70)) \gtrsim 1.4 \times 10^{42} \mathrm{erg} \mathrm{s}^{-1}$, and a calibration is given in Equation (22). Similarly, $L(160)$ is linearly correlated with the SFR for luminosities $L(160)>10^{42} \mathrm{erg} \mathrm{s}^{-1}$ (Equation (25)).

\subsection{Scatter and the Impact of Metallicity}

Both calibrations, however, have significant scatter, $\sigma_{70} \sim$ 0.2 dex and $\sigma_{160} \sim 0.4 \mathrm{dex}$, around the mean trend, and larger than the scatter, $\sim 0.12-0.16$ dex, of calibrations based on $L(24)$ or a mix of $L(24)$ and $L(\mathrm{H} \alpha)_{\text {obs }}$ (Rieke et al. 2009; Kennicutt et al. 2009). Furthermore, the scatter increases for increasing wavelength, and appears to decrease for increasing luminosity (Figures 9 and 15). To further investigate these trends, we subdivide the high-metallicity sample into two subsamples of oxygen abundance higher and lower than $12+\log (\mathrm{O} / \mathrm{H})=8.5$. This separating value is chosen after Figure 5 of Engelbracht et al. (2008), which shows that galaxies above a metallicity of $12+\log (\mathrm{O} / \mathrm{H})=8.5$ tend to have a roughly constant $f_{v}(70) /$ $f_{v}(160)$ flux ratio (albeit with a large scatter), while galaxies below that metallicity value tend to have an increasing ratio for decreasing oxygen abundance. Figure 19 shows the highmetallicity data from our sample divided into the two subsamples. The scatter of the data for both $\Sigma_{70}$ and $\Sigma_{160}$ at constant $\Sigma_{\text {SFR }}$ decreases when the sample is restricted to the highest metallicity data $(12+\log (\mathrm{O} / \mathrm{H})>8.5)$; specifically, faint $\Sigma_{70}$ and $\Sigma_{160}$ galaxies at fixed $\Sigma_{\mathrm{SFR}}$ drop from the subsample. This is in the expected direction, as seen earlier in this paper when analyzing the impact of low-metallicity galaxies (Figure 12).

The results of Figure 19 should be considered preliminary at this stage, since the oxygen abundances for a number of high-metallicity galaxies are uncertain (sometimes by about 0.2 dex). Nevertheless, they confirm and support the results of Section 5 for SFR(70), in the sense that as the galaxy metallicity increases and its dispersion in the sample decreases, $\Sigma_{70}$ tends to approach a one-to-one relation with $\Sigma_{\mathrm{SFR}}$ (Figure 19, left). Conversely, in the same metallicity range, $\Sigma_{160}$ tends to show a flattening in its relation with $\Sigma_{\mathrm{SFR}}$ and the dispersion of the data points around the mean trend remains large (Figure 19, right). As already discussed in Section 6, this is in agreement with the expectation that the emission at long infrared wavelengths receives an increasing contribution (and an increasing scatter) from dust heated by evolved stellar populations unassociated with the current star formation event (Lonsdale Persson \& Helou 1987). Figure 19 (right) further stresses that the calibration of SFR(160), Equation (25), should only be used for $L(160) \gtrsim$ $2 \times 10^{42} \mathrm{erg} \mathrm{s}^{-1}$, equivalent to $\Sigma(160) \gtrsim 10^{41} \mathrm{erg} \mathrm{s}^{-1} \mathrm{kpc}^{-2}$.

The calibrations just quoted apply to galaxies with mean gasphase metallicity $12+\log (\mathrm{O} / \mathrm{H}) \gtrsim 8.1$. The scatter becomes even larger, and strongly asymmetric, when galaxies of lower oxygen abundance value are included (Figure 12). Indeed, the general expectation is that lower-metallicity galaxies will in general contain less dust and be more transparent than highmetallicity ones. In this case, the infrared will be a poor tracer of the current SFR, as most of the light produced by recently formed stars will emerge unabsorbed by dust. Thus, calibrations of SFR(70) and SFR(160) need to be applied with an awareness of these limitations.

A hybrid approach, which combines, e.g., $L(70)$ with $L(\mathrm{H} \alpha)_{\text {obs }}$, could likely provide a "remedy" for the dependence of far-infrared SFR indicators on metallicity, similarly to what has been already verified for $L(24)$ and $L(8)$, where the $\mathrm{H} \alpha$ observed luminosity accounts for the unabsorbed portion of the SFR (Calzetti et al. 2007; Kennicutt et al. 2007, 2009; Zhu et al. 2008). However, our current data do not enable us to test such hybrid indicators. We already use, in our analysis, the combination of $L(24)$ and $L(\mathrm{H} \alpha)_{\text {obs }}$ to trace SFR in galaxies in an unbiased fashion; this indicator is dominated by the $\mathrm{H} \alpha$ luminosity at low SFSDs, as would be any hybrid combination of $L(70)$ and $L(\mathrm{H} \alpha)_{\mathrm{obs}}$, thus resulting in a degeneracy for any test we may attempt. 


\subsection{Limits of Validity for the Calibrations}

For the high-metallicity galaxies, the presence of a contribution to the long-wavelength infrared emission from dust heated by populations unassociated with the current star formation becomes more evident for decreasing galaxy luminosity (Figures 11 and 15), but it also competes with at least three more effects: (1) the effective dust temperature as measured from the IR SED tends to decrease (Figures 4, 5, and 17); (2) the overall ISM becomes progressively more transparent, thus a lower fraction of the SFR is traced in the infrared; and (3) the area filling factor of star-forming regions within galaxies decreases down to $\sim 10 \%-50 \%$ (depending on the dust extinction and stellar population models adopted), contributing to a change in the proportionality between $\Sigma_{70}$ or $\Sigma_{160}$ with $\Sigma_{\text {SFR }}$ relative to that of more luminous galaxies. These four effects all contribute, at various levels, to change the relation between $\Sigma_{70}$ (or $\Sigma_{160}$ ) with $\Sigma_{\text {SFR }}$ from linear to nonlinear (e.g., Equation (21)), thus complicating any SFR calibration for luminosities below $L(70) \sim$ $1.4 \times 10^{42} \mathrm{erg} \mathrm{s}^{-1}\left(L(160) \sim 2 \times 10^{42} \mathrm{erg} \mathrm{s}^{-1}\right)$. These luminosity values correspond to SFR $\sim 0.1-0.3 M_{\odot} \mathrm{yr}^{-1}$, thus they are not extremely restrictive especially in the context of current studies of distant galaxy populations.

\subsection{Additional Uncertainties}

So far, we have not considered another potential source of heating for the dust associated with the diffuse ISM of galaxies: photons leaking out of $\mathrm{H}$ II regions. About 20\%-50\% of the integrated $\mathrm{H} \alpha$ luminosity in galaxies is associated with the diffuse ISM (Reynolds 1990; Ferguson et al. 1996; Wang et al. 1997; Martin 1997), and those photons will produce dust heating. Since photons leaking out of $\mathrm{H}$ II regions are associated with the current star formation, in our analysis we have assumed that the diffuse ionized gas follows the same scaling relations as the star-forming regions, albeit possibly with different absolute values (e.g., the diffuse photons may heat the dust to typically lower temperatures than those spatially associated with $\mathrm{H}$ II regions). This assumption is justified by the findings of Wang et al. (1998), according to which the fraction of diffuse-to-total ionized gas remains relatively constant in galaxies of increasing SFR, from normal star forming to starbursts, once the diffuse gas surface brightness is normalized by the mean SFR per unit area of the galaxy.

The infrared emission from the galaxies in our sample is dominated by dust heated by current star formation, with, in some cases, a non-negligible contribution from evolved stellar populations. Central active galactic nuclei (AGNs) do not constitute a dominant heating source in the present sample, and their impact on our calibrations could not be investigated. Thus, the application of Equation (22) (or 25) to galaxy populations first requires the evaluation of any contamination from dust heating by AGNs.

Finally, all our derivations are predicated on the assumption that a universal stellar IMF can be applied to all galaxies. While this is generally considered a reasonable assumption, recent investigations using the Sloan Digital Sky Survey galaxy sample (Hoverstern \& Glazebrook 2008), a complete sample of nearby galaxies (Lee et al. 2009), and H I-selected galaxies (Meurer et al. 2009) suggest that the galaxy-integrated IMF may become increasingly bottom heavy in low luminosity and/or low surface brightness galaxies. Changes in the IMF will clearly impact the calibration of SFR indicators across the full electromagnetic wavelength range, and establishing whether such variations are present and what physical parameters may be causing them is an avenue of future investigation.

This work is based in part on observations made with the Spitzer Space Telescope, which is operated by the Jet Propulsion Laboratory, California Institute of Technology under a contract with NASA. This work is part of LVL and SINGS, two of the Spitzer Space Telescope Legacy Science Programs; it has been partially supported by the JPL, Caltech, Contract Number 1316765. A.G.d.P. is partially financed by the Spanish Ramón y Cajal program and the Programa Nacional de Astronoma y Astrofisica under grant AYA 2006-02358. C.-N. Hao acknowledges the support from NSFC key project 10833006 . We thank an anonymous referee for many comments that have helped improve the manuscript. This research has made use of the NASA/IPAC Extragalactic Database (NED) which is operated by the Jet Propulsion Laboratory, California Institute of Technology, under contract with the National Aeronautics and Space Administration.

\section{REFERENCES}

Alonso-Herrero, A., Engelbracht, C. W., Rieke, M. J., Rieke, G. H., \& Quillen, A. C. 2001, ApJ, 546, 952

Alonso-Herrero, A., Rieke, G. H., Rieke, M. J., Colina, L., Perez-Gonzalez, P. G., \& Ryder, S. D. 2006, ApJ, 650, 835

Alonso-Herrero, A., Rieke, G. H., Rieke, M. J., \& Scoville, N. Z. 2000, ApJ, 532,845

Alonso-Herrero, A., Rieke, G. H., Rieke, M. J., \& Scoville, N. Z. 2002, AJ, 124, 166

Bell, E. F. 2003, ApJ, 586, 794

Bendo, G. J., et al. 2008, MNRAS, 389, 629

Bigiel, F., Leroy, A., Walter, F., Brinks, E., de Blok, W. J. G., Madore, B., \& Thornley, M. D. 2008, AJ, 136, 2846

Boselli, A., \& Gavazzi, G. 2002, A\&A, 386, 124

Boselli, A., Lequeux, J., \& Gavazzi, G. 2004, A\&A, 428, 409

Bouchet, P., Lequeux, J., Maurice, E., Prevot, L., \& Prevot-Burnichon, M. L. 1985, A\&A, 149, 330

Braine, J., \& Dumke, M. 1998, A\&A, 333, 38

Buat, V., Boselli, A., Gavazzi, G., \& Bonfanti, C. 2002, A\&A, 383, 801

Buat, V., \& Xu, C. 1996, A\&A, 306, 61

Buat, V., et al. 2005, ApJ, 619, L51

Buat, V., et al. 2007, ApJS, 173, 404

Calzetti, D. 2001, PASP, 113, 1449

Calzetti, D., Armus, L., Bohlin, R. C., Kinney, A. L., Koornneef, J., \& StorchiBergmann, T. 2000, ApJ, 533, 682

Calzetti, D., Conselice, J. C., Gallagher, J. S., \& Kinney, A. L. 1999, AJ, 118, 797

Calzetti, D., \& Kennicutt, K. C. 2009, PASP, 121, 937

Calzetti, D., Kinney, A. L., \& Storchi-Bergmann, T. 1994, ApJ, 429, 582

Calzetti, D., et al. 2005, ApJ, 633, 871

Calzetti, D., et al. 2007, ApJ, 666, 870

Cannon, J. M., et al. 2005, ApJ, 630, L37

Cannon, J. M., et al. 2006a, ApJ, 647, 293

Cannon, J. M., et al. 2006b, ApJ, 652, 1170

Chabrier, G. 2003, PASP, 115, 763

Chanial, P., Flores, H., Guiderdoni, B., Elbaz, D., Hammer, F., \& Vigroux, L. 2007, A\&A, 462, 81

Croft, S., et al. 2006, ApJ, 647, 1040

Dale, D. A., \& Helou, G. 2002, ApJ, 576, 159

Dale, D. A., et al. 2005, ApJ, 633, 857

Dale, D. A., et al. 2007, ApJ, 655, 863

Dale, D. A., et al. 2009, ApJ, 703, 517

DeVaucouleurs, G., De Vaucouleurs, A., Corwin, Jr., H. G., Buta, R. J., Paturel, G., \& Fouque, P. 1991, Third Reference Catalogue of Bright Galaxies, Version 3.9

Devereux, N. A., \& Young, J. S. 1990, ApJ, 350, L25

Draine, B. T., \& Li, A. 2007, ApJ, 657, 810

Draine, B. T., et al. 2007, ApJ, 633, 866

Dunne, L., \& Eales, S. 2001, MNRAS, 327, 697

Dunne, L., Eales, S., Edmunds, M., Ivison, R., Alexander, P., \& Clements, D. L. 2000, MNRAS, 315, 115 
Engelbracht, C. W., Gordon, K. D., Rieke, G. H., Werner, M. W., Dale, D. A., \& Latter, W. B. 2005, ApJ, 628, 29

Engelbracht, C. W., et al. 2008, ApJ, 685, 678

Evans, I. N., Koratkar, A. P., Storchi-Bergmann, T., Kirkpatrick, H., Heckman, T. M., \& Wilson, A. S. 1996, ApJS, 105, 93

Fabbiano, G., Heckman, T., \& Keel, W. C. 1990, ApJ, 355, 442

Ferguson, A. M. N., Wyse, R. F. G., Gallagher, J. S., \& Hunter, D. A. 1996, AJ, 111,2265

Flaherty, K. M., Pipher, J. L., Megeath, E. M., Winston, E. M., Gutermuth, R. A., Muzerolle, J., Allen, L. E., \& Fazio, G. G. 2007, ApJ, 663, 1069

Förster Schreiber, N. M., Roussel, H., Sauvage, M., \& Charmandaris, V. 2004, A\&A, 419, 501

Garcia-Barreto, J. A., \& Rosado, M. 2001, AJ, 121, 2540

Genzel, R., et al. 1998, ApJ, 498, 579

Gil de Paz, A., Madore, B. F., \& Pevunova, O. 2003, ApJS, 147, 29

Goldader, J. D., Meurer, G., Heckman, T. M., Seibert, M., Sanders, D. B., Calzetti, D., \& Steidel, C. C. 2002, ApJ, 568, 651

Haas, M., Klaas, U., \& Bianchi, S. 2002, A\&A, 385, L23

Hattori, T., et al. 2004, AJ, 127, 736

Heckman, T. M., Robert, C., Leitherer, C., Garnett, D. R., \& van der Rydt, F. 1998, ApJ, 503, 646

Helou, G. 1986, ApJ, 311, L33

Hernandez, O., Carignan, C., Amram, P., Chemin, L., \& Daigle, O. 2005, MNRAS, 360, 1201

Hernandez-Toledo, H. M., Fuentes-Carrera, I., Rosado, M., Cruz-Gonzalez, I., Franco-Balderas, A., \& Dultzin-Hacyan, D. 2003, A\&A, 412, 669

Hirashita, H., Buat, V., \& Inoue, A. K. 2003, A\&A, 410, 83

Hopkins, A. M., Connolly, A. J., Haarsma, D. B., \& Cram, L. E. 2001, AJ, 122 , 288

Hoverstern, E. A., \& Glazebrook, K. 2008, ApJ, 675, 163

Hunter, D. A., \& Elmegreen, B. G. 2004, AJ, 128, 2170

Hunter, D. A., Gallagher, J. S., Rice, W. L., \& Gillett, F. C. 1989, ApJ, 336, 152

Hunter, D. A., Gillett, F. C., Gallagher, J. S., Rice, W. L., \& Low, F. J. 1986, ApJ, 303,171

Hunter, D. A., van Woerden, H., \& Gallaher, J. S. 1994, ApJS, 91, 79

Jackson, D. C., Cannon, J. M., Skillman, E. D., Lee, H., Gehrz, R. D., Woodward, C. E., \& Polomski, E. 2006, ApJ, 646, 192

James, P. A., et al. 2004, A\&A, 414, 23

Johnson, K. E., Leitherer, C., Vacca, W. D., \& Conti, P. S. 2000, AJ, 120, 1273

Kennicutt, R. C., Jr. 1998, ARA\&A, 36, 189

Kennicutt, R. C., Lee, J. C., Funes, S. J., Sakai, S., \& Akiyama, S. 2008, ApJS, 178,247

Kennicutt, R. C., Roettiger, K. A., Keel, W. C., van der Hulst, J. M., \& Hummel, E. 1987, AJ, 93, 1011

Kennicutt, R. C., et al. 2003, PASP, 115, 928

Kennicutt, R. C., et al. 2007, ApJ, 671, 333

Kennicutt, R. C., et al. 2009, ApJ, 703, 1672

Kewley, L. J., Geller, M. J., \& Jansen, R. A. 2004, AJ, 127, 2002

Kewley, L. J., Geller, M. J., Jansen, R. A., \& Dopita, M. A. 2002, AJ, 124, 3135

Kniazev, A. Y., Pustilnik, S. A., Grebel, E. K., Lee, H., \& Pramskij, A. G. 2004, ApJS, 153, 429

Koopman, R. A., Kenney, J. D. P., \& Joung, J. 2001, ApJS, 135, 125

Kroupa, P. 2001, MNRAS, 322, 231

Larsen, S. S., \& Richtler, T. 1999, A\&A, 345, 59

Lee, J. C., et al. 2009, ApJ, 706, 599

Lehnert, M. D., \& Heckman, T. M. 1996, ApJ, 472, 546

Leitherer, C., et al. 1999, ApJS, 123, 3

Leroy, A., Walter, F., Brinks, E., Bigiel, F., de Blok, W. J. G., Madore, B., \& Thornley, M. D. 2008, AJ, 136, 2782
Lonsdale Persson, C. J., \& Helou, G. X. 1987, ApJ, 314, 513

Lonsdale, C. J., et al. 2003, PASP, 115, 897

Madden, et al. 2006, A\&A, 446, 877

Marble, A. R., et al. 2010, ApJ, submitted

Martin, C. L. 1997, ApJ, 491, 561

Mendez, D. I., Esteban, C., \& Balcells, M. 1999, AJ, 117, 1229

Meurer, G. R., Heckman, T. M., \& Calzetti, D. 1999, ApJ, 521, 64

Meurer, G. R., et al. 2009, ApJ, 695, 765

Moustakas, J., \& Kennicutt, R. C. 2006, ApJS, 164, 81

Moustakas, J., Kennicutt, R. C., \& Tremonti, C. A. 2006, ApJ, 642, 775

Nagao, T., Maiolino, R., \& Marconi, A. 2006, A\&A, 459, 85

Nishiyama, S., et al. 2009, ApJ, 696, 1407

Osterbrock, D. E., \& Ferland, G. J. 2006, Astrophysics of Gaseous Nebulae and Active Galactic Nuclei (2nd ed; Sausalito, CA: Univ. Science Books)

Pahre, M. A., Ashby, M. L. N., Fazio, G. G., \& Willner, S. P. 2004, ApJS, 154, 235

Perez-Gonzalez, P. G., et al. 2006, ApJ, 648, 987

Persic, M., \& Rephaeli, Y. 2007, A\&A, 463, 481

Ranalli, P., Comastri, A., \& Setti, G. 2003, A\&A, 399, 39

Relaño, M., Lisenfeld, U., Perez-Gonzalez, P. G., Vilchez, J. M., \& Battaner, E. 2007, ApJ, 667, L141

Reynolds, R. J. 1990, in IAU Symp. 139, The Galactic and Extragalactic Background Radiation, ed. S. Bowyer \& C. Leinert (Dordrecht: Kluwer), 157

Rieke, G. H., Alonso-Herrero, A., Weiner, B. J., Perez-Gonzalez, P. G., Blaylock, M., Donley, J. L., \& Marcillac, D. 2009, ApJ, 692, 556

Robitaille, T. P., Rossa, J., Bomans, D. J., \& van der Marel, R. P. 2007, A\&A, 464,541

Rosa-Gonzalez, D., Burgarella, D., Nandra, K., Kunth, D., Terlevich, E., \& Terlevich, R. 2007, MNRAS, 379, 357

Rosenberg, J. L., Ashby, M. L. N., Salzer, J. J., \& Huang, J.-S. 2006, ApJ, 636, 742

Roussel, H., Sauvage, M., Vigroux, L., \& Bosma, A. 2001, A\&A, 372, 427

Rowan-Robinson, M., \& Crawford, J. 1989, MNRAS, 238, 523

Salim, S., et al. 2007, ApJS, 173, 267

Salim, S., et al. 2009, ApJ, 700, 161

Salpeter, E. E. 1955, ApJ, 121, 161

Sanders, D. B., Mazzarella, J. M., Kim, D.-C., Surace, J. A., \& Soifer, B. T. 2003, AJ, 126, 1607

Sauvage, M., \& Thuan, T. X. 1992, ApJ, 396, L69

Schlegel, D. J., Finkbeiner, D. P., \& Davis, M. 1998, ApJ, 500, 525

Schmitt, H. R., Calzetti, D., Armus, L., Giavalisco, M., Heckman, T. M., Kennicutt, R. C., Leitherer, C., \& Meurer, G. R. 2006, ApJS, 164, 52

Seibert, M., et al. 2005, ApJ, 619, L23

Scoville, N. Z., et al. 2000, AJ, 119, 991

Surace, J. A., Sanders, D. B., \& Mazzarella, J. M. 2004, AJ, 127, 3235

Walter, F., et al. 2007, ApJ, 661, 102

Walterbos, R. A. M., \& Greenawalt, B. 1996, ApJ, 460, 696

Wang, B., \& Heckman, T. M. 1996, ApJ, 457, 645

Wang, J., Heckman, T. M., \& Lehnert, M. D. 1997, ApJ, 491, 114

Wang, J., Heckman, T. M., \& Lehnert, M. D. 1998, ApJ, 509, 93

Wang, J., Heckman, T. M., \& Lehnert, M. D. 1999, ApJ, 515, 97

Wang, Z., \& Helou, G. 1992, ApJ, 398, L33

Wu, H., Cao, C., Hao, C.-N., Liu, F.-S., Wang, J.-L., Xia, X.-Y., Deng, Z.-G., \& Young, C. K.-S. 2005, ApJ, 632, L79

Wu, Y., Charmandaris, V., Hao, L., Brandl, B. R., Bernard-Salas, J., Spoon, H. W. W., \& Houck, J. R. 2006, ApJ, 639, 157

Yun, M. S., Reddy, N. A., \& Condon, J. J. 2001, ApJ, 554, 803

Zhu, Y.-N., Wu, H., Cao, C., \& Li, H.-N. 2008, ApJ, 686, 155 